\title{
ASYMPTOTICS OF THE SPECTRUM OF THE HYDROGEN ATOM IN A MAGNETIC FIELD NEAR THE LOWER BOUNDARIES OF SPECTRAL CLUSTERS
}

\author{
A. V. PERESKOKOV
}

\begin{abstract}
We investigate the second-order Zeeman effect in a magnetic field using irreducible representations of an algebra with the Karasev - Novikova quadratic commutation relations. To each such representation there corresponds a spectral cluster near the energy level of the unperturbed hydrogen atom. Using this model as an example, we describe a general method for constructing asymptotic solutions near the boundaries of spectral clusters based on a new integral representation. We also study the problem of computing quantum averages near the lower boundaries of clusters.
\end{abstract}

\section{INTRODUCTION}

A non-relativistic Hamiltonian for the hydrogen atom in a homogeneous magnetic field has the form

$$
\mathbb{H}=\mathbb{H}_{0}+\varepsilon \mathbb{M}_{3}+\varepsilon^{2} \mathbb{W}
$$

where

$$
\mathbb{H}=-\Delta-|x|^{-1}, \quad \mathbb{M}_{3}=i x_{2} \frac{\partial}{\partial x_{1}}-i x_{1} \frac{\partial}{\partial x_{2}}, \quad \mathbb{W}=\left(x_{1}^{2}+x_{2}^{2}\right) / 4 .
$$

Here, $x=\left(x_{1}, x_{2}, x_{3}\right)$ denotes the Cartesian coordinates in $\mathbb{R}^{3}, \Delta$ is the Laplace operator, the magnetic field is in the direction of the $x_{3}$-axis, and the parameter $\varepsilon$ in (1) is proportional to the magnetic field strength. The study of the hydrogen atom in a magnetic field is of great interest both in physics and mathematics, with a lot of papers devoted to it (see, for example, [1] $[$ [] $)$.

An algebraic method for constructing the asymptotics of the spectrum and the eigenfunctions of the operator (1) was introduced in [9. It is based on an algebraic averaging of the perturbation, followed by the passage to the symmetry algebra, and a coherent transformation from the original representation of this algebra to an irreducible representation of that algebra in the space of functions over a Lagrangian submanifold in a symplectic leaf. Later on, this method was extended to a wide class of problems with frequency resonances [10, 11. It has been worked through on a number of physical models [12 - 14.

Of special interest are the states of the system (1) corresponding to the boundaries of spectral clusters near the eigenvalues of the unperturbed operator, where the above Lagrangian submanifolds almost collapse and the integral representation of the solution over them becomes impossible. A possible approach to constructing asymptotics near

\footnotetext{
2010 Mathematics Subject Classification. Primary 81R30; Secondary 34Mxx, 81Q20, 33Cxx.

Key words and phrases. Operator averaging method, coherent transformation, WKB approximation, turning point, spectral cluster.

The author was partially supported by the RFFI (Project 12-01-00627-a), the Ministry of Education and Science of the RF (Contract 14.V37.21.0864), and the Grant Council of the President of the RF (Project NSh-2033.2012.1).
} 
cluster boundaries using "deformed" coherent states was outlined in [9, but so far it has not been justified in higher approximations.

Using the spectral problem for a two-dimensional perturbed oscillator as an example, we proposed, in [15 and [16, another method for finding the series of asymptotic eigenvalues near the boundaries of spectral clusters. It is based on a new integral representation for the corresponding asymptotic eigenfunctions. In the present paper, we apply this method to find the asymptotics of the spectrum of the hydrogen atom in a magnetic field near the lower boundaries of spectral clusters (see Theorems 5 and 8 for asymptotic formulas for eigenvalues and eigenfunctions). We want to emphasize that the obtained asymptotic formula for the eigenfunction is global. It cannot be derived by such standard tools as the ray method [17] or the complex germ theory [18.

We now describe the plan of the paper. In $\S 2$, we regularize the Hamiltonian (1); $\S 3$ and $\S 4$ describe the quantum averaging method and the coherent transformation in the case of an algebra with the Karasev - Novikova commutation relations. In $\S 5$, we find an integral representation for the asymptotic eigenfunctions which are antiholomorphic polynomials of degree $n-|m|-1$. In $\S 6, \S 7$, and $\S 8$, we consider the multipoint spectral problem. We construct an asymptotic solution and compute the correction in the spectral series. In $\S 9$, we study the asymptotic eigenfunctions and compare their asymptotics with the WKB approximation. In $\S 10$, we find the asymptotics of the norm. Finally, $\S 11$ contains the proofs of the summarizing theorems. We also obtain there formulas for quantum averages.

\section{Regularization}

Fix an integer $m$. In $L^{2}\left(\mathbb{R}^{3}\right)$, consider the eigenvalue problem for the Hamiltonian (1) and for the third component of the angular momentum:

$$
\left(\mathbb{H}_{0}+\varepsilon^{2} \mathbb{W}\right) \psi=E \psi, \quad \mathbb{M}_{3} \psi=m \psi
$$

Following [9, we regularize the Hamiltonian (1). For each $n \geq 1$ and $E<0$, we introduce parameters $\nu$ and $\mu$, a new variable $q \in \mathbb{R}^{3}$, and a function $\tilde{\psi}$ by the formulas

$$
E=-\frac{1}{4 n^{2} \nu^{2}}, \quad \mu=\varepsilon^{2} n^{6} \nu^{4}, \quad q=\frac{x}{n^{2} \nu}, \quad \psi(x)=\frac{\tilde{\psi}(q)}{n^{2}} .
$$

Furthermore, set $\hbar=1 / n$ and consider the operators

$$
\boldsymbol{S}_{0}=|q|\left(\frac{1}{4}+\left(-i \hbar \frac{\partial}{\partial q}\right)^{2}\right), \quad \boldsymbol{S}_{1}=\frac{|q|}{4}\left(q_{1}^{2}+q_{2}^{2}\right), \quad \boldsymbol{M}_{3}=q_{1}\left(-i \hbar \frac{\partial}{\partial q_{2}}\right)-q_{2}\left(-i \hbar \frac{\partial}{\partial q_{1}}\right) .
$$

The substitution formula (3) yields the spectral problem

$$
\left(\boldsymbol{S}_{0}+\mu \boldsymbol{S}_{1}\right) \tilde{\psi}=\nu \tilde{\psi}, \quad \boldsymbol{M}_{3} \tilde{\psi}=\hbar m \tilde{\psi}
$$

in the Hilbert space $L_{-}^{2}\left(\mathbb{R}^{3}\right)$ with scalar product

$$
\left\langle\varphi, \varphi^{\prime}\right\rangle_{-}=\frac{\pi}{4} \int_{\mathbb{R}^{3}} \frac{\varphi(q) \bar{\varphi}^{\prime}(q)}{|q|} d q .
$$

The number $\nu$ in (4) is the desired eigenvalue, and $\mu \geq 0$ is a parameter. The problem (2) on the subspace corresponding to the negative spectrum $E<0$ is equivalent to the spectral problem (4) in $L_{-}^{2}\left(\mathbb{R}^{3}\right)$.

Notice that in the absence of the magnetic field, the spectrum of the operator $\boldsymbol{S}_{0}$ is integral $\{\hbar N: N=1,2,3, \ldots\}$. In particular, $\boldsymbol{S}_{0}$ has the eigenvalue $\hbar n=1$. For $\mu>0$, we consider the branches of the eigenvalues of the operator $\boldsymbol{S}_{0}+\mu \boldsymbol{S}_{1}$ equal to 1 in the limit when $\mu=0$. They have the form $1+\mu \lambda_{k, m, n}(\mu)$, where $\lambda_{k, m, n}(\mu)$ are some smooth functions in a neighborhood of $\mu=0$ and $k$ is the branch number. The corresponding eigenfunctions of the problem (4) will be denoted by $\tilde{\psi}_{k, m, n}(q, \mu)$. (For brevity, we shall 
omit the numbers $m$ and $n$ in the indices.) After finding the functions $\lambda_{k}(\mu)$ from (4), we can use the system

$$
1+\mu \lambda_{k}(\mu)=\nu, \quad \mu=\varepsilon^{2} n^{6} \nu^{4}
$$

to determine $\nu=\nu_{k}(\varepsilon)$ and $\mu=\mu_{k}(\varepsilon)$.

\section{Algebraic averaging}

Suppose that $\varepsilon^{2} n^{7} \lesssim 1$ and $\nu$ is of order 1 . Then $\mu \ll 1$ and we can apply a quantum version of the averaging method [19], 20, 9] to the problem (4). The basic idea of this method is to find an invertible operator $\boldsymbol{U}$ and an operator $\underline{\boldsymbol{S}_{1}}+\mu \underline{\boldsymbol{S}_{2}}$ such that

$$
\begin{gathered}
\boldsymbol{U}^{-1}\left(\boldsymbol{S}_{0}+\mu \boldsymbol{S}_{1}\right) \boldsymbol{U}=\boldsymbol{S}_{0}+\mu \underline{\boldsymbol{S}_{1}}+\mu^{2} \underline{\boldsymbol{S}_{2}}+O\left(\mu^{3}\right), \\
{\left[\underline{\boldsymbol{S}_{1}}+\mu \underline{\boldsymbol{S}_{2}}, \boldsymbol{S}_{0}\right]=\left[\underline{\boldsymbol{S}_{1}}+\mu \underline{\boldsymbol{S}_{2}}, \boldsymbol{M}_{3}\right]=0 .}
\end{gathered}
$$

The new perturbation operator $\boldsymbol{S}_{1}+\mu \boldsymbol{S}_{2}$ commutes with the leading part of $\boldsymbol{S}_{0}$ and with the operator $\boldsymbol{M}_{3}$. Hence the spectral problem (4) reduces to the same problem for the operator $\underline{\boldsymbol{S}_{1}}+\mu \underline{\boldsymbol{S}_{2}}$ on $L[m, n] \subset L_{-}^{2}\left(\mathbb{R}^{3}\right)$, the subspace of simultaneous eigenfunctions of the operators $\boldsymbol{S}_{0}$ and $\boldsymbol{M}_{3}$. Since

$$
\nu=1+\mu \lambda(\mu), \quad \tilde{\psi}(q, \mu)=\boldsymbol{U} \varphi(q, \mu),
$$

the averaging problem has the form

$$
\begin{gathered}
\boldsymbol{S}_{0} \varphi=\varphi, \quad \boldsymbol{M}_{3} \varphi=\hbar m \varphi, \\
\left(\underline{\boldsymbol{S}_{1}}+\mu \underline{\boldsymbol{S}_{2}}+O\left(\mu^{2}\right)\right) \varphi=\lambda \varphi .
\end{gathered}
$$

We remark that this idea can be realized only under rather strong restrictions on $\boldsymbol{S}_{0}$. More precisely, one has to have

$$
\exp \left\{\frac{2 \pi i}{\hbar} \boldsymbol{S}_{0}\right\}=I
$$

where $I$ is the identity operator. Since in our problem the spectrum of the operator $\boldsymbol{S}_{0}$ is integral, the condition (10) is satisfied.

The operator $\boldsymbol{U}$ can be explicitly computed [9]. Define

$$
\boldsymbol{S}_{1}^{\#}=\frac{1}{2 \pi} \int_{0}^{2 \pi}(\pi-\tau) \exp \left\{-\frac{i \tau}{\hbar} \boldsymbol{S}_{0}\right\} \boldsymbol{S}_{1} \exp \left\{\frac{i \tau}{\hbar} \boldsymbol{S}_{0}\right\} d \tau
$$

Then

$$
\boldsymbol{U}=\exp \left\{-i \mu n \boldsymbol{S}_{1}^{\#}\right\}+O\left(\varepsilon^{2} n^{6}\right) .
$$

The transformation (11) is called the de-averaging transformation. It allows one to pass to approximate eigenfunctions of the original (non-averaged) problem.

The solution of the problem (8), (9) can be written in the form $\varphi=\varphi_{k}+O\left(\varepsilon^{2} n^{6}\right)$, where $\left\{\varphi_{k}(q)\right\}$ is an orthonormal basis consisting of the eigenfunctions $\underline{\boldsymbol{S}_{1}}$ corresponding to some eigenvalues $\xi_{k}$ :

$$
\boldsymbol{S}_{0} \varphi_{k}=\varphi_{k}, \quad \boldsymbol{M}_{3} \varphi_{k}=\hbar m \varphi_{k}, \quad \underline{\boldsymbol{S}_{1}} \varphi_{k}=\xi_{k} \varphi_{k} .
$$

The eigenvalues $\lambda=\lambda_{k}(\mu)$ of the problem (8), (9) are of the form

$$
\lambda_{k}(\mu)=\xi_{k}+\mu \eta_{k}+O\left(\mu^{2}\right),
$$

where

$$
\eta_{k}=\left\langle\underline{\boldsymbol{S}_{2}} \varphi_{k}, \varphi_{k}\right\rangle_{-}
$$


Solving the system (5) approximately, we have

$$
\begin{gathered}
\nu_{k}(\varepsilon)=1+\varepsilon^{2} n^{6} \xi_{k}+\varepsilon^{4} n^{12}\left(\eta_{k}+4 \xi_{k}^{2}\right)+O\left(\varepsilon^{6} n^{18}\right), \\
\mu_{k}(\varepsilon)=\varepsilon^{2} n^{6}+4 \varepsilon^{4} n^{12} \xi_{k}+O\left(\varepsilon^{6} n^{18}\right) .
\end{gathered}
$$

Substituting these expressions in (3), (7), and (11), we have that the asymptotics of the solution of the original problem (2) is given by the formulas (see [9])

$$
\begin{gathered}
E_{k}=-\frac{1}{4 n^{2}}+\frac{1}{2} \varepsilon^{2} n^{4} \xi_{k}+\frac{1}{4} \varepsilon^{4} n^{10}\left(2 \eta_{k}+5 \xi_{k}^{2}\right)+O\left(\varepsilon^{6} n^{16}\right), \\
\psi_{k}(x)=\frac{1}{n^{2}} \exp \left\{-i \varepsilon^{2} n^{7} \boldsymbol{S}_{1}^{\#}\right\} \varphi_{k}+O\left(\varepsilon^{2} n^{6}\right),
\end{gathered}
$$

where the functions in the right-hand side of (15) are taken at the point $x /\left(n^{2} \nu_{k}(\varepsilon)\right)$.

Consider the Karasev - Novikova algebra $\mathcal{F}_{\text {quant }}$ consisting of the first integrals of the pair $\boldsymbol{S}_{0}, \boldsymbol{M}_{3}$ [9], 21]. Its generators $\boldsymbol{B}_{0}, \boldsymbol{B}_{1}, \boldsymbol{B}_{2}, \boldsymbol{B}_{3}$ are subject to the following quadratic commutation relations: uadratic commutation relations:

$$
\begin{gathered}
{\left[\boldsymbol{B}_{1}, \boldsymbol{B}_{2}\right]=i \hbar \boldsymbol{B}_{0} \boldsymbol{B}_{3}, \quad\left[\boldsymbol{B}_{0}, \boldsymbol{B}_{1}\right]=2 i \hbar \boldsymbol{B}_{2},} \\
{\left[\boldsymbol{B}_{2}, \boldsymbol{B}_{3}\right]=-\frac{i \hbar}{2}\left(\boldsymbol{B}_{0} \boldsymbol{B}_{1}+\boldsymbol{B}_{1} \boldsymbol{B}_{0}\right), \quad\left[\boldsymbol{B}_{0}, \boldsymbol{B}_{2}\right]=-2 i \hbar \boldsymbol{B}_{1},} \\
{\left[\boldsymbol{B}_{3}, \boldsymbol{B}_{1}\right]=-\frac{i \hbar}{2}\left(\boldsymbol{B}_{0} \boldsymbol{B}_{2}+\boldsymbol{B}_{2} \boldsymbol{B}_{0}\right), \quad\left[\boldsymbol{B}_{0}, \boldsymbol{B}_{3}\right]=0 .}
\end{gathered}
$$

By (6), the averaged Hamiltonians $\underline{\boldsymbol{S}_{1}}, \underline{\boldsymbol{S}_{2}}$ can be expressed via the generators $\boldsymbol{B}=$ $\left(\boldsymbol{B}_{0}, \boldsymbol{B}_{1}, \boldsymbol{B}_{2}, \boldsymbol{B}_{3}\right)$ of $\mathcal{F}_{\text {quant }}$. We have (see [9])

\section{Lemma 1.}

$$
\underline{\boldsymbol{S}_{1}}=\boldsymbol{S}_{0} g_{0}(\boldsymbol{B}), \quad \underline{\boldsymbol{S}_{2}}=\boldsymbol{S}_{0} g_{00}(\boldsymbol{B}),
$$

where the operators $\boldsymbol{B}_{0}, \boldsymbol{B}_{1}, \boldsymbol{B}_{2}, \boldsymbol{B}_{3}$ are Weyl-symmetrized, and

$$
\begin{gathered}
g_{0}(b)=12 b_{3}-8 b_{2}-4 M_{3}^{2}+4 \hbar^{2}, \\
g_{00}(b)=-\frac{1}{3}\left(4\left(108 b_{2}^{2}+239 b_{3}^{2}-308 b_{2} b_{3}\right)+4\left(-66 S_{0}^{2}+100 M_{3}^{2}-234 \hbar^{2}\right) b_{2}\right. \\
+4\left(65 S_{0}^{2}-130 M_{3}^{2}+249 \hbar^{2}\right) b_{3}-127 S_{0}^{2} M_{3}^{2}+72 M_{3}^{4}+127 \hbar^{2} S_{0}^{2} \\
\left.\quad-277 \hbar^{2} M_{3}^{2}+205 \hbar^{4}\right) .
\end{gathered}
$$

\section{Coherent transformation}

Suppose $m$ and $n$ are integers such that $n>|m| \geq 0$. Let $\mathcal{P}[m, n]$ denote the space of antiholomorphic polynomials over $\mathbb{C}$ of degree at most $n-|m|-1$, with scalar product given by

$$
\left(\Phi_{1}, \Phi_{2}\right)_{\mathcal{P}[m, n]}=\int_{\mathbb{C}} \Phi_{1}(\bar{z}) \overline{\Phi_{2}(\bar{z})} d \mu_{m, n}(z) .
$$

Here $d \mu_{m, n}(z)=\varrho\left(|z|^{2}\right) d \bar{z} d z$,

$$
\varrho(r)=\frac{n(n-|m|)(|m|+1)_{n}}{2 \pi(n+1)_{n+1}} r^{|m|} F(n+1, n+|m|+1 ; 2 n+2 ; 1-r),
$$

where $F$ is a hypergeometric series (see [22]), and the operation $(l)_{M}$ is given by

$$
(l)_{M} \equiv l(l+1) \ldots(l+M-1), \quad(l)_{0} \equiv 1 .
$$


Notice that the function $\varrho(r)$ is a unique solution of the following problem:

$$
\begin{gathered}
r(1-r) \varrho^{\prime \prime}+((1-|m|)-(2 n-|m|+3) r) \varrho^{\prime}-(n+1)(n-|m|+1) \varrho=0, \\
\int_{0}^{\infty} \varrho(r) d r=\frac{1}{2 \pi}, \quad \varrho(r)>0 .
\end{gathered}
$$

To solve problem (12), we use the coherent transformation (see [23], [9], 21])

$$
\mathfrak{H}(\Phi)=\int_{\mathbb{C}} \Phi(\bar{z}) \mathfrak{H}_{z} d \mu_{m, n}(z)
$$

Here, the hypergeometric coherent states have the form

$$
\mathfrak{H}_{z}=\sum_{j=0}^{n-|m|-1} \overline{P_{j}(\bar{z})} \chi_{j}
$$

where

$$
P_{j}(\bar{z})=\sqrt{k_{j}} \bar{z}^{j}, \quad k_{j}=\frac{(n-j)_{j}(n-|m|-j)_{j}}{j !(1+|m|)_{j}}, \quad j=0, \ldots, n-|m|-1,
$$

is an orthonormal basis in $\mathcal{P}[m, n]$, and

$$
\begin{aligned}
\chi_{j}(q)=c_{j}\left(q_{1}+i \operatorname{sgn}(m) q_{2}\right)^{|m|} & \\
& \times \exp \left(-\frac{|q|}{2 \hbar}\right) L_{j}^{|m|}\left(\frac{|q|+q_{3}}{2 \hbar}\right) L_{n-|m|-1-j}^{|m|}\left(\frac{|q|-q_{3}}{2 \hbar}\right) .
\end{aligned}
$$

In the formula (25), $L_{N}^{M}(y)$ are the Laguerre polynomials (see 22]), and the normalizing constants $c_{j}$ have the form

$$
c_{j}=(-1)^{j} /\left(2^{|m|} \pi \hbar^{|m|+1} \sqrt{\left.(n-|m|-j)_{|m|}(1+j)_{|m|}\right)} .\right.
$$

The coherent transformation (23) maps the Hilbert space $\mathcal{P}[m, n]$ to the Hilbert space $L[m, n]$. The transformation $\mathfrak{H}$ is unitary, and its inverse $\mathfrak{H}^{-1}: L[m, n] \rightarrow \mathcal{P}[m, n]$ can be computed as

$$
\mathfrak{H}^{-1}(\chi)=\left\langle\chi, \mathfrak{H}_{z}\right\rangle_{L[m, n]} .
$$

Notice that the integral representation

$$
\varphi_{k}=\mathfrak{H}\left(\Phi_{k}\right)
$$

yields exact solutions of the first two equations (12) for all amplitudes $\Phi_{k}$. Since, under the coherent transformation $(23), \boldsymbol{B}_{0}, \boldsymbol{B}_{1}, \boldsymbol{B}_{2}, \boldsymbol{B}_{3}$ turn into differential operators of the first and second orders:

$$
\begin{gathered}
\stackrel{\circ}{B}_{0}=\hbar\left(2 \bar{z} \frac{d}{d \bar{z}}-n+|m|+1\right), \\
\stackrel{\circ}{B}_{1}=\frac{i \hbar^{2}}{2}\left(\bar{z}\left(\bar{z}^{2}-1\right) \frac{d^{2}}{d \bar{z}^{2}}-\left((2 n-|m|-3) \bar{z}^{2}+|m|+1\right) \frac{d}{d \bar{z}}+(n-1)(n-|m|-1) \bar{z}\right), \\
\stackrel{\circ}{B}_{2}=\frac{\hbar^{2}}{2}\left(\bar{z}\left(\bar{z}^{2}+1\right) \frac{d^{2}}{d \bar{z}^{2}}-\left((2 n-|m|-3) \bar{z}^{2}-|m|-1\right) \frac{d}{d \bar{z}}+(n-1)(n-|m|-1) \bar{z}\right), \\
\stackrel{\circ}{B}_{3}=-\hbar^{2}\left(\bar{z}^{2} \frac{d^{2}}{d \bar{z}^{2}}-(n-|m|-2) \bar{z} \frac{d}{d \bar{z}}-\frac{1}{2}(n-1)(|m|+1)\right),
\end{gathered}
$$

we have, by (17) and (12), the following equation for $\Phi_{k}$ :

$$
\left(12 \stackrel{\circ}{B}_{3}-8 \stackrel{\circ}{B}_{2}-4 \hbar^{2} m^{2}+4 \hbar^{2}\right) \Phi_{k}=\xi_{k} \Phi_{k} .
$$

By the eigenvalues of the equation (28) we shall understand the values of the parameter $\xi_{k}$ such that this equation has polynomial solutions in $\mathcal{P}[m, n]$. We shall require that

$$
\left\|\Phi_{k}\right\|_{\mathcal{P}[m, n]}=1 .
$$


Let us show how the point $\bar{z}=1$ is related to the spectrum of the operator $g_{0}(\boldsymbol{B})$. Set $c=|m| / n$ and let

$$
\begin{gathered}
n \rightarrow \infty, \quad|m| \rightarrow \infty, \\
5^{-1 / 2}<c<1 .
\end{gathered}
$$

Consider the restriction of the function (17) to the symplectic leaf $\Omega$ of the algebra (16) given by the equations

$$
b_{3}^{2}-b_{1}^{2}-b_{2}^{2}=c^{2} / 4, \quad b_{0}^{2}+4 b_{3}=c^{2}+1 .
$$

Define a Kähler structure on $\Omega$ by the complex coordinate

$$
z=\frac{2\left(b_{3}+i b_{1}\right)}{1-2 b_{3}-b_{0}} .
$$

Let $\bar{z}$ be the function complex-conjugate to (33). Then, by (32) and (33) (see [9]),

$$
\begin{gathered}
b_{0}=\frac{(c-1)\left(-2|z|^{2}-c\left(|z|^{2}-1\right)+\sqrt{\varkappa\left(|z|^{2}\right)}\right)}{2|z|^{2}-c\left(|z|^{2}-1\right)+\sqrt{\varkappa\left(|z|^{2}\right)}}, \\
b_{3}=\frac{c^{2}+1-b_{0}^{2}}{4}=\frac{c}{2}+\frac{2(c-1)^{2}|z|^{2}}{c\left(|z|^{2}-1\right)^{2}+4|z|^{2}+\left(|z|^{2}+1\right) \sqrt{\varkappa\left(|z|^{2}\right)}} \\
b_{2}=\frac{(z+\bar{z})}{4}\left(1-2 b_{3}-b_{0}\right)=\frac{(z+\bar{z})(1-c)\left(c\left(|z|^{2}+1\right)+\sqrt{\varkappa\left(|z|^{2}\right)}\right)}{2\left(c\left(|z|^{2}-1\right)^{2}+4|z|^{2}+\left(|z|^{2}+1\right) \sqrt{\varkappa\left(|z|^{2}\right)}\right)}, \\
b_{1}=\frac{(z-\bar{z}) b_{2}}{i(z+\bar{z})}=\frac{(z-\bar{z})(1-c)\left(c\left(|z|^{2}+1\right)+\sqrt{\varkappa\left(|z|^{2}\right)}\right)}{2 i\left(c\left(|z|^{2}-1\right)^{2}+4|z|^{2}+\left(|z|^{2}+1\right) \sqrt{\varkappa\left(|z|^{2}\right)}\right.} .
\end{gathered}
$$

Here

$$
\varkappa(r)=c^{2}(r-1)^{2}+4 r .
$$

Now, in $g_{0}$, we change the coordinates $b_{0}, b_{1}, b_{2}, b_{3}$ to $z, \bar{z}$ [9, 21]. As a result, the restriction of $g_{0}$ to $\Omega$ becomes

$$
g_{0, \Omega}(z, \bar{z})=6 c-4 c^{2}+4 \hbar^{2}+\frac{4(c-1)\left[6(c-1)|z|^{2}+(z+\bar{z})\left(c\left(|z|^{2}+1\right)+\sqrt{\varkappa\left(|z|^{2}\right)}\right)\right]}{c\left(|z|^{2}-1\right)^{2}+4|z|^{2}+\left(|z|^{2}+1\right) \sqrt{\varkappa\left(|z|^{2}\right)}},
$$

where $\varkappa(r)$ has the form $(38)$.

Theorem 1. The global minimum of the function $g_{0, \Omega}(z, \bar{z})$ under the conditions (30) and (31) is attained at $z=\bar{z}=1$. It equals

$$
g_{0, \Omega}(1,1)=1+c^{2}+O\left(\hbar^{2}\right) .
$$

Proof. Suppose $z=x+i y, \bar{z}=x-i y$. Then $g_{0, \Omega}$ can be written in the form

$$
g_{0, \Omega}(x, y)=g_{1}\left(x^{2}+y^{2}\right)+2 x g_{2}\left(x^{2}+y^{2}\right),
$$

where

$$
\begin{gathered}
g_{1}(r)=6 c-4 c^{2}+4 \hbar^{2}+\frac{24(c-1)^{2} r}{c(r-1)^{2}+4 r+(r+1) \sqrt{\varkappa(r)}}, \\
g_{2}(r)=\frac{4(c-1)[c(r+1)+\sqrt{\varkappa(r)}]}{c(r-1)^{2}+4 r+(r+1) \sqrt{\varkappa(r)}} .
\end{gathered}
$$


The critical points of $g_{0, \Omega}$ satisfy the system of equations

$$
\begin{gathered}
\frac{\partial g_{0, \Omega}(x, y)}{\partial x}=2\left[x g_{1}^{\prime}\left(x^{2}+y^{2}\right)+2 x^{2} g_{2}^{\prime}\left(x^{2}+y^{2}\right)+g_{2}\left(x^{2}+y^{2}\right)\right]=0 \\
\frac{\partial g_{0, \Omega}(x, y)}{\partial y}=2 y\left[g_{1}^{\prime}\left(x^{2}+y^{2}\right)+2 x g_{2}^{\prime}\left(x^{2}+y^{2}\right)\right]=0
\end{gathered}
$$

If $y \neq 0$ and $x \neq \infty$, we solve (40) for $x$ and substitute the result in (39). We then have an equation

$$
g_{2}\left(x^{2}+y^{2}\right)=0
$$

which has no solutions.

If $y=0$, then $x$ is a solution of the equation

$$
x g_{1}^{\prime}\left(x^{2}\right)+2 x^{2} g_{2}^{\prime}\left(x^{2}\right)+g_{2}\left(x^{2}\right)=0 .
$$

Since

$$
\begin{aligned}
g_{1}^{\prime}(r)=- & \frac{24(c-1)^{2}(r-1)\left[c^{2}\left(r^{2}+1\right)+2 r+c(r+1) \sqrt{\varkappa(r)}\right]}{\sqrt{\varkappa(r)}\left[c(r-1)^{2}+4 r+(r+1) \sqrt{\varkappa(r)}\right]^{2}}, \\
g_{2}(r)+2 r g_{2}^{\prime}(r)=- & 8(c-1)(r-1) \\
& \times \frac{c(r+1)\left[c^{2}\left(r^{2}+1\right)-2(c-2) r\right]+\left[c^{2}(r+1)^{2}-2(c-1) r\right] \sqrt{\varkappa(r)}}{\sqrt{\varkappa(r)}\left[c(r-1)^{2}+4 r+(r+1) \sqrt{\varkappa(r)}\right]^{2}},
\end{aligned}
$$

the equation (41) rewrites as

$$
g_{3}(x)\left(x^{2}-1\right)=0
$$

where

$$
\begin{aligned}
g_{3}(x)= & 3(c-1) x\left[c^{2}\left(x^{4}+1\right)+2 x^{2}+c\left(x^{2}+1\right) \sqrt{\varkappa\left(x^{2}\right)}\right] \\
& +c\left(x^{2}+1\right)\left[c^{2}\left(x^{4}+1\right)-2(c-2) x^{2}\right]+\left[c^{2}\left(x^{2}+1\right)^{2}-2(c-1) x^{2}\right] \sqrt{\varkappa\left(x^{2}\right)} .
\end{aligned}
$$

The solutions of (42) are $x= \pm 1$. We shall now show that under the condition (31), this equation has no other solutions. Indeed, when $x \leq 0$, the function $g_{3}(x)$ is positive because the first summand in (43) is nonnegative and the remaining summands are positive.

In the case $x>0$, the substitution $u=x+1 / x$ turns the equation

$$
g_{3}(x)=0
$$

into

$$
\begin{aligned}
{\left[c^{2} u^{2}+3 c(c-1) u\right.} & -2(c-1)] \sqrt{c^{2}\left(u^{2}-4\right)+4} \\
& =-c^{3} u^{3}-3 c^{2}(c-1) u^{2}+c\left(2 c^{2}+2 c-4\right) u+6\left(c^{2}-1\right)(c-1) .
\end{aligned}
$$

Squaring both sides of (45), we have a new equation,

$$
u^{2}+6 u-5 c^{-2}+9=0,
$$

whose solutions $u_{ \pm}=-3 \pm \sqrt{5} c^{-1}$ are less than 2 . Hence the equation

$$
x+1 / x=u_{ \pm}
$$

and, therefore, equation (44) has no solutions when $x>0$.

Let us check that the sufficient conditions for a local minimum hold at $z=\bar{z}=1$. Since

$$
\frac{\partial^{2} g_{0, \Omega}(1,0)}{\partial x \partial y}=0
$$


it suffices to show that

$$
\begin{gathered}
\frac{\partial^{2} g_{0, \Omega}(1,0)}{\partial x^{2}}=2 g_{1}^{\prime}(1)+12 g_{2}^{\prime}(1)+4 g_{1}^{\prime \prime}(1)+8 g_{2}^{\prime \prime}(1)>0, \\
\frac{\partial^{2} g_{0, \Omega}(1,0)}{\partial y^{2}}=2 g_{1}^{\prime}(1)+4 g_{2}^{\prime}(1)>0 .
\end{gathered}
$$

Since

$$
g_{1}^{\prime}(1)=0, \quad g_{2}^{\prime}(1)=\frac{1-c^{2}}{2}, \quad g_{1}^{\prime \prime}(1)=-\frac{3}{8}\left(1-c^{2}\right)^{2}, \quad g_{2}^{\prime \prime}(1)=\frac{\left(c^{2}-1\right)\left(5-c^{2}\right)}{8},
$$

it follows from (31) that

$$
\frac{\partial^{2} g_{0, \Omega}(1,0)}{\partial x^{2}}=\frac{\left(1-c^{2}\right)\left(5 c^{2}-1\right)}{2}>0, \quad \frac{\partial^{2} g_{0, \Omega}(1,0)}{\partial y^{2}}=2\left(1-c^{2}\right)>0,
$$

and therefore $g_{0, \Omega}$ has a local minimum at $z=\bar{z}=1$. Since the values

$$
\begin{gathered}
\left.g_{0, \Omega}\right|_{z=\bar{z}=1}=c^{2}+1+4 \hbar^{2},\left.\quad g_{0, \Omega}\right|_{z=\bar{z}=-1}=-3 c^{2}+5+4 \hbar^{2}, \\
\left.g_{0, \Omega}\right|_{z=\bar{z}=\infty}=6 c-4 c^{2}+4 \hbar^{2}
\end{gathered}
$$

satisfy the inequalities

$$
c^{2}+1<6 c-4 c^{2}<-3 c^{2}+5,
$$

$g_{0, \Omega}$ has a global minimum at this point.

The number $g_{0, \Omega}(1,1)$ determines the lower boundary of the spectral cluster. Later on, we shall compute the correction for this number (see formula (105)).

In addition to the spectrum problem, consider also the problem of finding the average values of differential operators on the solutions of (2) near the boundaries of spectral clusters. Quantum averages play an important role in applications and can be found experimentally. Notice that for

$$
\xi_{k} \sim 1+c^{2}
$$

the equation

$$
g_{0, \Omega}(z, \bar{z}) \varphi_{k}=\xi_{k} \varphi_{k},
$$

to which (4) reduces on the eigenspace $L[m, n]$, will have the eigenfunctions $\varphi_{k}$ localized in a small neighborhood of $z=\bar{z}=1$. Therefore, the formula for quantum averages becomes

$$
\left(F\left(b_{0}, b_{1}, b_{2}, b_{3}\right) \varphi_{k}, \varphi_{k}\right) \sim F\left(0,0,\left(1-c^{2}\right) / 4,\left(1+c^{2}\right) / 4\right)\left(\varphi_{k}, \varphi_{k}\right) .
$$

Here, the functions $b_{0}, b_{1}, b_{2}, b_{3}$ were approximated by their values at $z=\bar{z}=1$, computed by the formulas (34)-(37). Moreover,

$$
F\left(0,0,\left(1-c^{2}\right) / 4,\left(1+c^{2}\right) / 4\right) \neq 0 .
$$

A rigorous justification of the above arguments will be given later (see Theorem 6).

\section{INTEGRAL REPRESENTATION FOR ASYMPTOTIC EIGENFUNCTIONS}

Consider equation (28). In view of (27), we have

$$
\begin{aligned}
& \frac{R(\bar{z})}{|m|^{2}} \frac{d^{2} \Phi_{k}}{d \bar{z}^{2}}-\frac{1}{|m|}\left[\left(2 \sqrt{a}-1-\frac{3}{|m|}\right) \bar{z}^{2}+3\left(\sqrt{a}-1-\frac{2}{|m|}\right) \bar{z}-1-\frac{1}{|m|}\right] \frac{d \Phi_{k}}{d \bar{z}} \\
& \quad+\left[(\sqrt{a}-1)\left(\sqrt{a}-1-\frac{1}{|m|}\right) \bar{z}-\frac{1}{2}\left(1+\frac{1}{|m|}\right)\left(3 \sqrt{a}-2-\frac{1}{|m|}\right)+\frac{\tilde{\xi}_{k}}{4}\right] \Phi_{k}=0
\end{aligned}
$$


where

$$
\begin{gathered}
R(\bar{z})=\bar{z}\left(\bar{z}^{2}+3 \bar{z}+1\right), \\
a=\frac{n^{2}}{|m|^{2}}=\frac{1}{c^{2}}, \quad \tilde{\xi}_{k}=\frac{n^{2}}{|m|^{2}} \xi_{k} .
\end{gathered}
$$

(To simplify notation, we shall drop the subscript $k$ of the function $\Phi$.) This equation has three singular points:

$$
\bar{z}_{1}=0, \quad \bar{z}_{2}=(-3+\sqrt{5}) / 2, \quad \bar{z}_{3}=(-3-\sqrt{5}) / 2,
$$

which are solutions of the equation $R(\bar{z})=0$, and the singular point $\bar{z}_{4}=\infty$. Since all four singular points are regular, (48) is a Fuchsian equation [24, [25]. Fuchsian equations with four singular points are called Heun equations.

Fuchsian equations with three singular points give rise to the well-known systems of classical orthogonal polynomials [22. No such theory exists for Fuchsian equations with four or more singular points. As was mentioned in [25], there are only sporadic cases when one can construct polynomial solutions for Heun equations. In the present paper we shall determine asymptotic eigenvalues and the corresponding eigenfunctions (polynomials) for the problems (48), (29) under the conditions (30), (31).

We will need a number of results from the theory of coherent transformations [21. Together with $\mathcal{P}[m, n]$, consider the dual Hilbert space $\widetilde{\mathcal{P}}[m, n]$, consisting of meromorphic distributions on $\mathbb{C} /\{0\}$ of the form

$$
\tilde{g}(z)=\sum_{j=0}^{n-|m|-1} \frac{\tilde{g}_{j}}{z^{j+1}} .
$$

The norm in $\widetilde{\mathcal{P}}[m, n]$ is given by

$$
\|\tilde{g}\|_{\widetilde{\mathcal{P}}[m, n]}^{2}=\frac{1}{(2 \pi)^{2}} \oint_{\gamma} \oint_{\gamma} K(\bar{w}, z) \tilde{g}(z) \overline{\tilde{g}(\bar{w})} d z d \bar{w}
$$

where the contour integrals are computed over the cycles $\gamma$ around $z=0$ oriented counterclockwise, and

$$
K(\bar{w}, z)=\sum_{j=0}^{n-|m|-1} k_{j}(z \bar{w})^{j}
$$

is the reproducing kernel. The constants $k_{j}$ in (52) are given by (24). The following result can be found in 21 .

Lemma 2. The duality between $\widetilde{\mathcal{P}}[m, n]$ and $\mathcal{P}[m, n]$ is given by the map

$$
\mathcal{K}: \tilde{\mathcal{P}}[m, n] \rightarrow \mathcal{P}[m, n], \quad(\mathcal{K} \tilde{g})(\bar{w})=\frac{1}{2 \pi i} \oint_{\gamma} K(\bar{w}, z) \tilde{g}(z) d z .
$$

The inverse map is given by

$$
\left(\mathcal{K}^{-1} g\right)(z)=-\frac{1}{2 \pi i} \oint_{\gamma} \tilde{L}(\bar{w}, z) g(\bar{w}) d \bar{w}
$$

where

$$
\tilde{L}(\bar{w}, z)=\sum_{j=0}^{n-|m|-1} \frac{1}{k_{j}(\bar{w} z)^{j+1}}
$$

is the meromorphic reproducing kernel. 
Notice that in the case of the Heisenberg commutation relations, a variant of meromorphic coherent states and transformations similar to (53) and (54) were introduced by Dirac [26].

Let $G(\bar{u}, \bar{w})$ denote the superposition kernel of the operators $\mathcal{K}^{-1}$ and $\mathcal{K}$. It follows from (52) and (55) that

$$
\begin{aligned}
G(\bar{u}, \bar{w})=\frac{1}{2 \pi i} \oint_{\gamma} K(\bar{w}, z) \tilde{L}(\bar{u}, z) d z & \\
=\frac{1}{2 \pi i} \oint_{\gamma}\left(\sum_{j=0}^{n-|m|-1} k_{j}(z \bar{w})^{j}\right) & \left(\sum_{\ell=0}^{n-|m|-1} \frac{1}{k_{\ell}(\bar{u} z)^{\ell+1}}\right) d z \\
& =\frac{1}{\bar{u}} \sum_{j=0}^{n-|m|-1}\left(\frac{\bar{w}}{\bar{u}}\right)^{j}=\frac{\bar{u}^{n-|m|}-\bar{w}^{n-|m|}}{\bar{u}^{n-|m|}(\bar{u}-\bar{w})} .
\end{aligned}
$$

The kernel (56) gives rise to the identity operator on $\mathcal{P}[m, n]$, and on the set $J$ of functions antiholomorphic in a neighborhood of zero, it is a projector to the space $\mathcal{P}[m, n]$. Indeed, if

$$
g(\bar{z})=\sum_{j=0}^{\infty} g_{j} \bar{z}^{j} \in J
$$

then

$$
-\frac{1}{2 \pi i} \oint_{\gamma} G(\bar{u}, \bar{w}) g(\bar{u}) d \bar{u}=\sum_{j=0}^{n-|m|-1} g_{j} \bar{w}^{j} .
$$

We are looking for a solution of (48) in the form

$$
\Phi(\bar{z})=-\frac{1}{2 \pi i} \oint_{\gamma} G(\bar{u}, \bar{z}) p(\bar{u}) d \bar{u},
$$

where $p \in J$, and $G$ is given by (56). Under the map (57), the operators $\stackrel{\circ}{B}_{2}, \stackrel{\circ}{B}_{3}$ turn into operators $\widehat{B}_{2}, \widehat{B}_{3}: J \rightarrow J$, and the equation (48) into the equation

$$
\left(12 \widehat{B}_{3}-8 \widehat{B}_{2}-4 \hbar^{2} m^{2}+4 \hbar^{2}-\xi_{k}\right) p=0 .
$$

To compute $\widehat{B}_{2}$ and $\widehat{B}_{3}$, we use the representation

$$
\stackrel{\circ}{B}_{2}=\frac{\stackrel{\circ}{A}(\stackrel{\circ}{B}-\stackrel{\circ}{C})}{2}+\frac{\hbar}{4}(n+|m|+1)(\stackrel{\circ}{B}+\stackrel{\circ}{C}), \quad \stackrel{\circ}{B}_{3}=-(\stackrel{\circ}{A})^{2}+\frac{\hbar^{2}}{4}\left(n^{2}+m^{2}-1\right),
$$

where

$$
\stackrel{\circ}{A}=\hbar\left(\frac{\ell}{2}-\bar{z} \frac{d}{d \bar{z}}\right), \quad \stackrel{\circ}{B}=\hbar \bar{z}\left(\ell-\bar{z} \frac{d}{d \bar{z}}\right), \quad \stackrel{\circ}{C}=\hbar \frac{d}{d \bar{z}},
$$

and

$$
\ell=n-|m|-1 .
$$

By (30) and (31), $\ell \rightarrow \infty$. Notice that the operators (60) are related, via

$$
\stackrel{\circ}{\AA}=\stackrel{\circ}{S}_{3}, \quad \stackrel{\circ}{B}=\stackrel{\circ}{S}_{1}-i \stackrel{\circ}{S}_{2}, \quad \stackrel{\circ}{C}=\stackrel{\circ}{S}_{1}+i \stackrel{\circ}{S}_{2},
$$

to the generators $\stackrel{\circ}{S}_{1}, \stackrel{\circ}{S}_{2}, \stackrel{\circ}{S}_{3}$ of the algebra $\mathfrak{s u}(2)$, which satisfy the following cyclic commutation relations:

$$
\left[\stackrel{\circ}{S}_{1}, \stackrel{\circ}{S}_{2}\right]=i \hbar \stackrel{\circ}{S}_{3}, \quad\left[\stackrel{\circ}{S}_{2}, \stackrel{\circ}{S}_{3}\right]=i \hbar \stackrel{\circ}{S}_{1}, \quad\left[\stackrel{\circ}{S}_{3}, \stackrel{\circ}{S}_{1}\right]=i \hbar \stackrel{\circ}{S}_{2} .
$$

Under the map (57), the operators $\AA \stackrel{\circ}{B}, \stackrel{\circ}{C}$ turn into the operators $\widehat{A}, \widehat{B}, \widehat{C}: J \rightarrow J$ defined by

$$
\widehat{A}=\hbar\left(\frac{\ell}{2}-\bar{u} \frac{d}{d \bar{u}}\right), \quad \widehat{B}=\hbar \bar{u}\left(\ell-\bar{u} \frac{d}{d \bar{u}}\right), \quad \widehat{C}=\hbar\left(\frac{d}{d \bar{u}}-\frac{\bar{u}^{\ell}}{\ell !} \frac{d^{\ell+1}}{d \bar{u}^{\ell+1}}\right) .
$$


Finally, from (59) and (61), we have

Lemma 3. The operators $\widehat{B}_{2}$ and $\widehat{B}_{3}$ have the form

$$
\begin{gathered}
\widehat{B}_{2}=\frac{\hbar^{2}}{2}\left(\bar{u}\left(\bar{u}^{2}+1\right) \frac{d^{2}}{d \bar{u}^{2}}-\left((2 n-|m|-3) \bar{u}^{2}-|m|-1\right) \frac{d}{d \bar{u}}\right. \\
+(n-1)(n-|m|-1) \bar{u})-\frac{\hbar^{2}}{2}\left(\bar{u} \frac{d}{d \bar{u}}+|m|+1\right) \frac{\bar{u}^{\ell}}{\ell !} \frac{d^{\ell+1}}{d \bar{u}^{\ell+1}}, \\
\widehat{B}_{3}=-\hbar^{2}\left(\bar{u}^{2} \frac{d^{2}}{d \bar{u}^{2}}-(n-|m|-2) \bar{u} \frac{d}{d \bar{u}}-\frac{1}{2}(n-1)(|m|+1)\right) .
\end{gathered}
$$

Consider the neighborhood of zero defined by

$$
|\bar{u}|<\left|\bar{z}_{2}\right|-\delta,
$$

where $\bar{z}_{2}=(-3+\sqrt{5}) / \sqrt{2}$, and $\delta>0$ is a constant. In this neighborhood, the asymptotic solution of (48) is given by the WKB approximation [27, 28]

$$
\Phi^{W K B}(\bar{z})=t(\bar{z}) e^{\ell s(\bar{z})}\left(1+O\left(\ell^{-1}\right)\right), \quad \ell \rightarrow \infty .
$$

Here $s(\bar{z}), t(\bar{z})$ are antiholomorphic functions, and $t(\bar{z}) \neq 0$. After the substitution of $\Phi^{W K B}(\bar{u})$ in (58), we have a residual $W$ which, in the neighborhood (62), has the form

$$
W=O\left(\ell^{-2} \Phi^{W K B}\right)+\hat{r} \Phi^{W K B},
$$

where

$$
\hat{r}=4 \hbar^{2}\left(\bar{u} \frac{d}{d \bar{u}}+|m|+1\right) \frac{\bar{u}^{\ell}}{\ell !} \frac{d^{\ell+1}}{d \bar{u}^{\ell+1}} .
$$

The expression $O\left(\ell^{-2} \Phi^{W K B}\right)$ in (63) is an estimate of the residual of the WKB approximation when, in (58), $\widehat{B}_{2}$ and $\widehat{B}_{3}$ are replaced by $\stackrel{\circ}{B}_{2}$ and $\stackrel{\circ}{B}_{3}$, and $\hat{r} \Phi^{W K B}$ appears because $\widehat{B}_{2}$ has an extra summand

$$
-\frac{\hbar^{2}}{2}\left(\bar{u} \frac{d}{d \bar{u}}+|m|+1\right) \frac{\bar{u}^{\ell}}{\ell !} \frac{d^{\ell+1}}{d \bar{u}^{\ell+1}} .
$$

We shall prove that $\hat{r} \Phi^{W K B}$ yields an exponentially small contribution as compared with $\ell^{-2} \Phi^{W K B}$.

Theorem 2. In a sufficiently small neighborhood of zero,

$$
\hat{r} \Phi^{W K B}=O\left(\ell^{-\infty} \Phi^{W K B}\right), \quad \ell \rightarrow \infty .
$$

Proof. Let $\gamma$ be the circle of radius $R_{0}<\left|\bar{z}_{2}\right|-\delta$ centered at zero. By the Cauchy integral formula, for $|\bar{u}|<R_{0}$, we have

$$
\hat{r} \Phi^{W K B}=-\frac{2(\ell+1)}{n^{2} \pi i} \oint_{\gamma} \frac{(n \bar{v}-(|m|-1) \bar{u}) \bar{u}^{\ell}}{(\bar{v}-\bar{u})^{\ell+3}} \Phi^{W K B}(\bar{v}) d \bar{v} .
$$

Applying the Cauchy inequalities [29] to (64) we have, for $|\bar{u}| \leq R_{0} / 2$, an estimate

$$
\left|\hat{r} \Phi^{W K B}(\bar{u})\right| \leq \frac{(\ell+1)(2 n+|m|-1) 2^{\ell+3}|\bar{u}|^{\ell}}{a|m|^{2} R_{0}^{\ell+1}} \max _{\bar{u} \in \gamma}|t(\bar{u})| \exp \left(\ell \max _{\bar{u} \in \gamma} \operatorname{Re} s(\bar{u})\right) .
$$

Furthermore, since $|t(\bar{u})|>0$ in the neighborhood (62), it follows from the maximum principle, when $|\bar{u}| \leq R_{0}$, that

$$
\left|\Phi^{W K B}(\bar{u})\right| \geq \min _{\bar{u} \in \gamma}|t(\bar{u})| \exp \left(\ell \min _{\bar{u} \in \gamma} \operatorname{Re} s(\bar{u})\right)>0 .
$$


Therefore, if $|\bar{u}| \leq R_{0} / 2$, then

$$
\begin{aligned}
\left|\frac{\hat{r} \Phi^{W K B}(\bar{u})}{\Phi^{W K B}(\bar{u})}\right| \leq \frac{(\sqrt{a}-1)(2 \sqrt{a}+1) 8}{a R_{0}} \frac{\max _{\bar{u} \in \gamma}|t(\bar{u})|}{\min _{\bar{u} \in \gamma}|t(\bar{u})|} \\
\quad \times \exp \left(\ell\left\{\ln |\bar{u}|-\ln R_{0}+\ln 2+\max _{\bar{u} \in \gamma} \operatorname{Re} s(\bar{u})-\min _{\bar{u} \in \gamma} \operatorname{Re} s(\bar{u})\right\}\right),
\end{aligned}
$$

and hence, for

$$
|\bar{u}|<R_{1}
$$

where

$$
0<R_{1}<\frac{R_{0}}{2} \exp \left(\min _{\bar{u} \in \gamma} \operatorname{Re} s(\bar{u})-\max _{\bar{u} \in \gamma} \operatorname{Re} s(\bar{u})\right),
$$

the right-hand side of (65) is exponentially small.

Thus, if in the formula (57) the cycle $\gamma$ around $\bar{u}=0$ lies in the domain (66), then the asymptotic solution of (58) of order $\ell+2$ can be replaced in the right-hand side of (57) by an asymptotic solution of (48) of order two.

\section{Multipoint SPECTRAL PRoblem}

Now we want to study the solutions of (48) near singular points. Using partial fractions, we have

$$
-\frac{|m|}{R(\bar{z})}\left[\left(2 \sqrt{a}-1-\frac{3}{|m|}\right) \bar{z}^{2}+3\left(\sqrt{a}-1-\frac{2}{|m|}\right) \bar{z}-1-\frac{1}{|m|}\right]=\sum_{j=1}^{3} \frac{A_{j}}{\bar{z}-\bar{z}_{j}},
$$

where $A_{1}=|m|+1, A_{2}=A_{3}=-n+1$. Solving the defining (or characteristic) equations

$$
\rho_{j}^{(i)}\left(\rho_{j}^{(i)}-1\right)+A_{j} \rho_{j}^{(i)}=0, \quad j=1,2,3 ; i=1,2,
$$

we find that the characteristic exponents at the singular points (51) are

$$
\rho_{j}^{(1)}=0, \quad j=1,2,3 ; \quad \rho_{1}^{(2)}=-|m|, \quad \rho_{j}^{(2)}=n, \quad j=2,3 .
$$

From the defining equation

$$
\rho_{\infty}^{(i)}\left(\rho_{\infty}^{(i)}-1\right)+(2 n-|m|-1) \rho_{\infty}^{(i)}+(n-1)(n-|m|-1)=0, \quad i=1,2,
$$

for the singular point $\bar{z}_{4}=\infty$, we find the characteristic exponents $\rho_{\infty}^{(1)}=-n+|m|+1$ and $\rho_{\infty}^{(2)}=-n+1$.

When $\tilde{\xi}=\tilde{\xi}_{k}$, the exact solutions of the spectral problem (48), (29) are polynomials from $\mathcal{P}[m, n]$. Their characteristic exponents at the singular points (51) are zero, and at $\bar{z}_{4}=\infty$ we have $\rho_{\infty}^{(1)}$. Notice that under the conditions (30), (31) the solutions with characteristic exponents $\rho_{j}^{(2)}, j=1,2,3,4$, do not belong to $\mathcal{P}[m, n]$ (and are the asymptotics of the polynomials from $\mathcal{P}[m, n]$ ). Indeed, for $j=2,3$, we have $\rho_{j}^{(2)}>$ $n-|m|-1$, and for $|m|>0$ we have $\rho_{1}^{(2)}<0$ and $-\rho_{\infty}^{(2)}>n-|m|-1$.

In the construction of asymptotic solutions of the equation (48), an important role is played by their behavior near singular points. Together with the spectral problem (48), (29), we consider a multipoint spectral problem. It consists of finding numbers $\tilde{\xi}_{k}$ (the eigenvalues) such that the equation (48) has nonzero antiholomorphic solutions whose characteristic exponents at the singular points (51) are zero, and at the singular point $\bar{z}_{4}=\infty$ the exponent is $-n+|m|+1$.

If a number $\tilde{\xi}_{k}$ and a function $p(\bar{u})$ are an asymptotic solution of such a multipoint spectral problem, then upon substituting $p(\bar{u})$ in the right-hand side of $(57)$, we have a polynomial $\Phi(\bar{z})$ which is an asymptotic solution of the equation (48) belonging to 
$\mathcal{P}[m, n]$. The normalizing condition $(29)$ for $\Phi(\bar{z})$ allows us to determine the arbitrary multiplier in $p(\bar{u})$. Thus, the number $\tilde{\xi}_{k}$ and the polynomial $\Phi(\bar{z})$ are an asymptotic solution of the original spectral problem (48), (29).

\section{The WKB approximation. The Stokes lines}

Let us construct asymptotic solutions of the equation (48). Using the substitution [24]

$$
\Phi(\bar{z})=E(\bar{z}) Y(\bar{z}),
$$

where

$$
E(\bar{z})=\left(\bar{z}^{2}+3 \bar{z}+1\right)^{(\sqrt{a}|m|-1) / 2}, \bar{z}^{-(|m|+1) / 2},
$$

we rewrite the equation (48) as

$$
\frac{d^{2} Y}{d \bar{z}^{2}}-Q(\bar{z}) Y=0
$$

\section{Lemma 4.}

$$
Q(\bar{z})=\frac{\left(|m|^{2}-1\right)}{4 R^{2}(\bar{z})}\left[\bar{z}^{4}+2 \bar{z}^{3}+\left(\frac{5\left(a-|m|^{-2}\right)}{1-|m|^{-2}}-1\right) \bar{z}^{2}+2 \bar{z}+1\right]-\frac{\tilde{\xi}_{k}|m|^{2}}{4 R(\bar{z})} .
$$

Since the conditions (30) are satisfied, it follows from (70) that

$$
Q(\bar{z})=\frac{|m|^{2} P(\bar{z})}{4 R^{2}(\bar{z})}-\frac{\tilde{\xi}_{k}|m|^{2}}{4 R(\bar{z})}+O(1)+O\left(\frac{1}{R^{2}(\bar{z})}\right) .
$$

Here $P(\bar{z})=\bar{z}^{4}+2 \bar{z}^{3}+(5 a-1) \bar{z}^{2}+2 \bar{z}+1$, and $R(\bar{z})$ is given by $(49)$.

Next we want to show how the existence of a multiple turning point $\bar{z}_{0}$ for the equation (69) allows us to find the asymptotics (46) for $\xi_{k}$. Consider the equations $Q\left(\bar{z}_{0}\right)=0$ and $Q^{\prime}\left(\bar{z}_{0}\right)=0$ up to $O(1)$. By $(71)$, we have

$$
P\left(\bar{z}_{0}\right)-\tilde{\xi}_{k} R\left(\bar{z}_{0}\right)=0, \quad P^{\prime}\left(\bar{z}_{0}\right)-\tilde{\xi}_{k} R^{\prime}\left(\bar{z}_{0}\right)=0 .
$$

This yields an equation for $\bar{z}_{0}$ :

$$
\begin{aligned}
{\left[\bar{z}_{0}^{4}+2 \bar{z}_{0}^{3}+(5 a-1) \bar{z}_{0}^{2}+2 \bar{z}_{0}\right.} & +1]\left[3 \bar{z}_{0}^{2}+6 \bar{z}_{0}+1\right] \\
& -\left[4 \bar{z}_{0}^{3}+6 \bar{z}_{0}^{2}+2(5 a-1) \bar{z}_{0}+2\right]\left[\bar{z}_{0}^{3}+3 \bar{z}_{0}^{2}+\bar{z}_{0}\right]=0
\end{aligned}
$$

and an asymptotics

$$
\tilde{\xi}_{k} \sim \frac{P\left(\bar{z}_{0}\right)}{R\left(\bar{z}_{0}\right)} .
$$

Factoring the left-hand side of (72),

$$
\left(\bar{z}_{0}^{2}-1\right)\left(\bar{z}_{0}^{2}+(3-\sqrt{5 a}) \bar{z}_{0}+1\right)\left(\bar{z}_{0}^{2}+(3+\sqrt{5 a}) \bar{z}_{0}+1\right)=0,
$$

we have that $(72)$ has six roots: $\bar{z}_{0,1}=1, \bar{z}_{0,2}=-1$,

$$
\begin{aligned}
& \bar{z}_{0,3}=\frac{-3+\sqrt{5 a}+5 i \sqrt{(\sqrt{5}-\sqrt{a})(\sqrt{a}-1 / \sqrt{5})}}{2}, \\
& \bar{z}_{0,4}=\frac{-3+\sqrt{5 a}-5 i \sqrt{(\sqrt{5}-\sqrt{a})(\sqrt{a}-1 / \sqrt{5})}}{2}, \\
& \bar{z}_{0,5}=\frac{-3-\sqrt{5 a}+\sqrt{5} \sqrt{(\sqrt{5}+\sqrt{a})(\sqrt{a}+1 / \sqrt{5})}}{2}, \\
& \bar{z}_{0,6}=\frac{-3-\sqrt{5 a}-\sqrt{5} \sqrt{(\sqrt{5}+\sqrt{a})(\sqrt{a}+1 / \sqrt{5})}}{2} .
\end{aligned}
$$


Setting $\bar{z}_{0}=1$ in $(73)$, we have

$$
\tilde{\xi}_{k} \sim a+1
$$

By (50), the asymptotics (46) now follows from (74). Notice that if in (73) we set $\bar{z}_{0}=-1$, then, for $a>1$, the number $\tilde{\xi}_{k} \sim 5 a-3$ will determine the upper boundary of the spectral cluster.

We will be looking for $\tilde{\xi}_{k}$ in the form

$$
\tilde{\xi}_{k}=a+1+\frac{\tilde{\xi}_{k}^{(1)}}{|m|}+O\left(\frac{1}{|m|^{2}}\right)
$$

where the numbers $\tilde{\xi}=\tilde{\xi}_{k}(k=0,1, \ldots)$ are in increasing order. Then $(69)$ rewrites as

$$
\frac{1}{|m|^{2}} \frac{d^{2} Y}{d \bar{z}^{2}}-\left(Q_{0}(\bar{z})-\frac{\tilde{\xi}_{k}^{(1)}}{|m| 4 R(\bar{z})}+O\left(\frac{1}{|m|^{2}}\right)+O\left(\frac{1}{|m|^{2} R^{2}(\bar{z})}\right)\right) Y=0 .
$$

Here $Q_{0}(\bar{z})=4^{-1}(\bar{z}-1)^{2} \Lambda(\bar{z}) R^{-2}(\bar{z})$, the polynomial $R(\bar{z})$ is given by (49), and

$$
\Lambda(\bar{z})=\bar{z}^{2}+(3-a) \bar{z}+1 .
$$

Setting $Q_{0}(\bar{z})$ equal to zero, we find that the equation (76) has a turning point $\bar{z}=1$ of order two and simple turning points

$$
\bar{z}_{ \pm}=(-3+a \pm i \sqrt{(a-1)(5-a)}) / 2 .
$$

They lie on the circle $|\bar{z}|=1$ and move on it from -1 to 1 when the parameter $a$ increases from 1 to 5 .

Let us construct the WKB approximations for the solutions of (76). They hold outside small neighborhoods of the turning points and are of the form

$$
\begin{aligned}
& Y_{ \pm}^{W K B}=\frac{\tilde{c}_{ \pm}}{\sqrt[4]{Q_{0}(\bar{z})}} \exp \left( \pm|m| \int \sqrt{Q_{0}(\bar{z})} d \bar{z} \mp \frac{\tilde{\xi}_{k}^{(1)}}{8} \int \frac{d \bar{z}}{R(\bar{z}) \sqrt{Q_{0}(\bar{z})}}\right)\left(1+O\left(\frac{1}{|m|}\right)\right. \\
& \left.\quad+O\left(\frac{1}{|m|(\bar{z}-1)^{2}}\right)+O\left(\frac{1}{|m|\left(\bar{z}-\bar{z}_{+}\right)^{3 / 2}}\right)+O\left(\frac{1}{|m|\left(\bar{z}-\bar{z}_{-}\right)^{3 / 2}}\right)\right), \quad|m| \rightarrow \infty .
\end{aligned}
$$

Here $\tilde{c}_{ \pm}$are constants, and, in the power functions, the principal values are taken. In addition, there is a cut along the circular arc $\smile \bar{z}_{-}, \bar{z}_{+}$connecting the points $\bar{z}_{-}, \bar{z}_{+}$and passing through -1 .

Let us compute the integrals appearing in (79).

Lemma 5. The WKB approximations $Y_{ \pm}^{W K B}$ can be represented as

$$
\begin{aligned}
Y_{ \pm}^{W K B}= & \frac{c_{ \pm}\left(\bar{z}^{2}+3 \bar{z}+1\right)^{ \pm n / 2+1 / 2}}{\sqrt{\bar{z}-1} \sqrt[4]{\Lambda(\bar{z})} \bar{z}^{ \pm|m| / 2-1 / 2}}\left(\frac{2 \sqrt{\Lambda(\bar{z})}+\sqrt{5-a}(\bar{z}+1)}{\bar{z}-1}\right)^{ \pm \tilde{\xi}_{k}^{(1)} /(4 \sqrt{5-a})} \\
& \times \frac{[\sqrt{\Lambda(\bar{z})}+\bar{z}+1]^{ \pm|m|}}{[\sqrt{\Lambda(\bar{z})}+\sqrt{a}(\bar{z}+1)]^{ \pm n}}\left(1+O\left(\frac{1}{|m|}\right)+O\left(\frac{1}{|m|(\bar{z}-1)^{2}}\right)\right. \\
& \left.\quad O\left(\frac{1}{|m|\left(\bar{z}-\bar{z}_{+}\right)^{3 / 2}}\right)+O\left(\frac{1}{|m|\left(\bar{z}-\bar{z}_{-}\right)^{3 / 2}}\right)\right), \quad|m| \rightarrow \infty
\end{aligned}
$$

Here $c_{ \pm}$are constants. 
Proof. We make use of the following integrals [30]:

$$
\begin{gathered}
\int \frac{d \bar{z}}{\bar{z} \sqrt{X(\bar{z})}}=-\frac{1}{\sqrt{c}} \ln \left(\frac{2 \sqrt{c X(\bar{z})}}{\bar{z}}+\frac{2 c}{\bar{z}}+b\right)+C \\
\int \frac{\sqrt{X(\bar{z})}}{\bar{z}} d \bar{z}=\sqrt{X(\bar{z})}+\frac{b}{2 \sqrt{a}} \ln (2 \sqrt{a X(\bar{z})}+2 a \bar{z}+b)+c \int \frac{d \bar{z}}{\bar{z} \sqrt{X(\bar{z})}},
\end{gathered}
$$

where $X(\bar{z})=a \bar{z}^{2}+b \bar{z}+c$, with constants $a>0, c>0$, and where $C$ is an arbitrary constant. From (81) we have

$$
\int \frac{d \bar{z}}{(\bar{z}-1) \sqrt{\bar{z}^{2}+(3-a) \bar{z}+1}}=-\frac{1}{\sqrt{5-a}} \ln \left(\frac{2 \sqrt{\Lambda(\bar{z})}+\sqrt{5-a}(\bar{z}+1)}{\bar{z}-1}\right)+C .
$$

Since

$$
\frac{\bar{z}-1}{R(\bar{z})}=\frac{1}{2}\left(-\frac{2}{\bar{z}}+\frac{1+\sqrt{5}}{\bar{z}-\bar{z}_{2}}+\frac{1-\sqrt{5}}{\bar{z}-\bar{z}_{3}}\right),
$$

it follows from (81) and (82) that

$$
\begin{aligned}
& \int \frac{(\bar{z}-1) \sqrt{\bar{z}^{2}+(3-a) \bar{z}+1}}{R(\bar{z})} d \bar{z}=\sqrt{\Lambda(\bar{z})}\left[-1+\frac{1+\sqrt{5}}{2}+\frac{1-\sqrt{5}}{2}\right] \\
& +\left[-\frac{3-a}{2}+\frac{(1+\sqrt{5})(\sqrt{5}-a)}{4}-\frac{(1-\sqrt{5})(\sqrt{5}+a)}{4}\right] \\
& \times \ln (2 \sqrt{\Lambda(\bar{z})}+2 \bar{z}+3-a)+\ln \left(\frac{2 \sqrt{\Lambda(\bar{z})}+2+(3-a) \bar{z}}{\bar{z}}\right) \\
& -\frac{(1+\sqrt{5})(\sqrt{5}-1) \sqrt{a}}{4} \ln \left(\frac{(\sqrt{5}-1) \sqrt{a} \sqrt{\Lambda(\bar{z})}+(\sqrt{5}-a) \bar{z}+(3-\sqrt{5})(a+\sqrt{5}) / 2}{\bar{z}-\bar{z}_{2}}\right) \\
& -\frac{(1-\sqrt{5})(\sqrt{5}+1) \sqrt{a}}{4} \ln \left(\frac{(\sqrt{5}+1) \sqrt{a} \sqrt{\Lambda(\bar{z})}-(\sqrt{5}+a) \bar{z}+(3+\sqrt{5})(a-\sqrt{5}) / 2}{\bar{z}-\bar{z}_{3}}\right)+C \\
& =\ln \left(\frac{(2 \sqrt{\Lambda(\bar{z})}+2 \bar{z}+3-a)(2 \sqrt{\Lambda(\bar{z})}+2+(3-a) \bar{z})}{\bar{z}}\right) \\
& -\frac{n}{|m|} \ln \left(\frac{(\sqrt{5}-1) \sqrt{a} \sqrt{\Lambda(\bar{z})}+(\sqrt{5}-a) \bar{z}+(3-\sqrt{5})(a+\sqrt{5}) / 2}{\bar{z}-\bar{z}_{2}}\right) \\
& -\frac{n}{|m|} \ln \left(\frac{(\sqrt{5}+1) \sqrt{a} \sqrt{\Lambda(\bar{z})}+(\sqrt{5}+a) \bar{z}+(3+\sqrt{5})(\sqrt{5}-a) / 2}{(5-a)(a-1)\left(\bar{z}-\bar{z}_{3}\right)}\right)+C \\
& =\ln \left(\frac{(5-a)(\sqrt{\Lambda(\bar{z})}+\bar{z}+1)^{2}}{\bar{z}}\right)-\frac{n}{|m|} \ln \left(\frac{(\sqrt{\Lambda(\bar{z})}+\sqrt{a}(\bar{z}+1))^{2}}{(a-1)\left(\bar{z}^{2}+3 \bar{z}+1\right)}\right)+C .
\end{aligned}
$$

Substituting the integrals (83) and (84) in (79), we have (80).

In the construction of asymptotic solutions in the complex plane, an important role is played by the Stokes lines [27, [31. Figure 1 shows, besides the singular points and the turning points, the Stokes lines for the equation (76).

The Stokes graph contains, in particular, the arc $\smile \bar{z}_{-}, \bar{z}_{+}$. To see that, we define a function

Since, by (80),

$$
W(\bar{z})=\frac{\left|\bar{z}^{2}+3 \bar{z}+1\right|^{\sqrt{a} / 2}|\sqrt{\Lambda(\bar{z})}+\bar{z}+1|}{|\bar{z}|^{1 / 2}|\sqrt{\Lambda(\bar{z})}+\sqrt{a}(\bar{z}+1)|^{\sqrt{a}}} .
$$

$$
W(\bar{z})=W\left(\bar{z}_{ \pm}\right) \exp \left(\operatorname{Re} \int_{\bar{z}_{ \pm}}^{\bar{z}} \sqrt{Q_{0}(\bar{z})} d \bar{z}\right)
$$




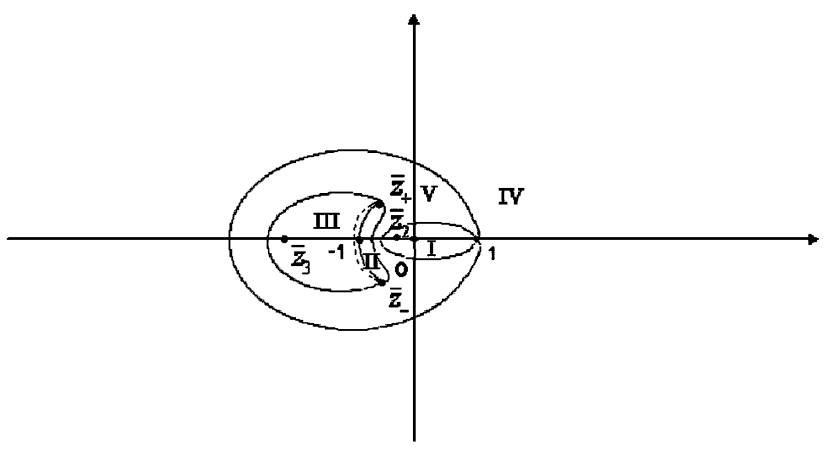

FiguRE 1

the Stokes lines passing through the turning points $\bar{z}_{ \pm}$are given by the equation

$$
W(\bar{z})=W\left(\bar{z}_{ \pm}\right),
$$

where $W\left(\bar{z}_{ \pm}\right)=(a-1)^{(\sqrt{a}-1) / 2}$. After the substitution $\tau=\bar{z} /(\bar{z}+1)^{2}$, the equation $(85)$ rewrites as

$$
\frac{|1+\tau|^{\sqrt{a} / 2}|\sqrt{1+(1-a) \tau}+1|}{|\tau|^{1 / 2}|\sqrt{1+(1-a) \tau}+\sqrt{a}|^{\sqrt{a}}}=(a-1)^{(\sqrt{a}-1) / 2} .
$$

If $\bar{z}$ moves along the arc $\smile \bar{z}_{-}, \bar{z}_{+}$from $\bar{z}_{-}$to $\bar{z}_{+}$, then the variable $\tau$ traverses the interval $[1 /(a-1),+\infty)$ twice. But since

$$
|\sqrt{1+(1-a) \tau}+1|=\sqrt{(a-1) \tau}, \quad|\sqrt{1+(1-a) \tau}+\sqrt{a}|=\sqrt{(a-1)(\tau+1)},
$$

the equation (86) holds on the arc $\smile \bar{z}_{-}, \bar{z}_{+}$.

Now we want to examine the behavior of the WKB approximations $Y_{ \pm}^{W K B}$ near the turning points. Taking the Taylor series of the functions in (79), we have

\section{Lemma 6.}

$$
\begin{aligned}
Y_{ \pm}^{W K B}=\frac{c_{ \pm}^{(+)}}{\sqrt[4]{\bar{z}-\bar{z}_{+}}} \exp \left( \pm|m| \tau_{+} \frac{2}{3}\left(\bar{z}-\bar{z}_{+}\right)^{3 / 2}\right)\left(1+O\left(|m|\left(\bar{z}-\bar{z}_{+}\right)^{5 / 2}\right)\right. \\
\left.+O\left(\sqrt{\bar{z}-\bar{z}_{+}}\right)+O\left(\frac{1}{|m|\left(\bar{z}-\bar{z}_{+}\right)^{3 / 2}}\right)\right)
\end{aligned}
$$

when $\bar{z} \rightarrow \bar{z}_{+},\left|\arg \left(\tau_{+}^{2 / 3}\left(\bar{z}-\bar{z}_{+}\right)\right)\right|<\pi$;

$$
\begin{aligned}
Y_{ \pm}^{W K B}=\frac{c_{ \pm}^{(-)}}{\sqrt[4]{\bar{z}-\bar{z}_{-}}} \exp \left( \pm|m| \tau_{-} \frac{2}{3}\left(\bar{z}-\bar{z}_{-}\right)^{3 / 2}\right)\left(1+O\left(|m|\left(\bar{z}-\bar{z}_{-}\right)^{5 / 2}\right)\right. \\
\left.+O\left(\sqrt{\bar{z}-\bar{z}_{-}}\right)+O\left(\frac{1}{|m|\left(\bar{z}-\bar{z}_{-}\right)^{3 / 2}}\right)\right)
\end{aligned}
$$

when $\bar{z} \rightarrow \bar{z}_{-},\left|\arg \left(\tau_{-}^{2 / 3}\left(\bar{z}-\bar{z}_{-}\right)\right)\right|<\pi$;

$$
\begin{aligned}
& Y_{ \pm}^{W K B}=c_{ \pm}^{(1)}(\bar{z}-1)^{-1 / 2} \mp \tilde{\xi}_{k}^{(1)} /(4 \sqrt{5-a}) \\
& \exp \left( \pm \frac{|m| \sqrt{5-a}}{20}\left[(\bar{z}-1)^{2}-(\bar{z}-1)^{3}\right]\right) \\
& \times\left(1+O(\bar{z}-1)+O\left(|m|(\bar{z}-1)^{4}\right)+O\left(\frac{1}{|m|(\bar{z}-1)^{2}}\right)\right)
\end{aligned}
$$

when $\bar{z} \rightarrow 1$. Here $c_{ \pm}^{(+)}, c_{ \pm}^{(-)}, c_{ \pm}^{(1)}$ are constants, and 
(90)

$$
\tau_{ \pm}=\frac{\left(\bar{z}_{ \pm}-1\right) \sqrt{\left|\bar{z}_{+}-\bar{z}_{-}\right|}}{2 \bar{z}_{ \pm}^{2} a} e^{ \pm i \pi / 4}=\frac{(a-5 \pm i \sqrt{(a-1)(5-a)}) \sqrt[4]{(a-1)(5-a)}}{4 \bar{z}_{ \pm}^{2} a} e^{ \pm i \pi / 4}
$$

Finally, we want to determine the WKB approximation for the solutions of (48). By (67), (68), and (80), we have

$$
\begin{aligned}
91) & \Phi_{-}^{W K B}=\frac{c_{-}}{\sqrt[4]{\left(\bar{z}-\bar{z}_{-}\right)\left(\bar{z}-\bar{z}_{+}\right)}}(\bar{z}-1)^{-1 / 2+\tilde{\xi}_{k}^{(1)} /(4 \sqrt{5-a})} \\
\times & (2 \sqrt{\Lambda(\bar{z})}+\sqrt{5-a}(\bar{z}+1))^{-\tilde{\xi}_{k}^{(1)} /(4 \sqrt{5-a})} \frac{[\sqrt{\Lambda(\bar{z})}+\sqrt{a}(\bar{z}+1)]^{n}}{[\sqrt{\Lambda(\bar{z})}+\bar{z}+1]^{|m|}} \\
\times(1+ & \left.O\left(\frac{1}{|m|}\right)+O\left(\frac{1}{|m|(\bar{z}-1)^{2}}\right)+O\left(\frac{1}{|m|\left(\bar{z}-\bar{z}_{+}\right)^{3 / 2}}\right)+O\left(\frac{1}{|m|\left(\bar{z}-\bar{z}_{-}\right)^{3 / 2}}\right)\right), \\
\Phi_{+}^{W K B}= & \frac{c_{+}(\bar{z}-1)^{-1 / 2-\tilde{\xi}_{k}^{(1)} /(4 \sqrt{5-a})}\left(\bar{z}^{2}+3 \bar{z}+1\right)^{n}}{\sqrt[4]{\left(\bar{z}-\bar{z}_{-}\right)\left(\bar{z}-\bar{z}_{+}\right)} \bar{z}^{|m|}} \\
& \times(2 \sqrt{\Lambda(\bar{z})}+\sqrt{5-a}(\bar{z}+1))^{\tilde{\xi}_{k}^{(1)} /(4 \sqrt{5-a})} \frac{[\sqrt{\Lambda(\bar{z})}+\bar{z}+1]^{|m|}}{[\sqrt{\Lambda(\bar{z})}+\sqrt{a}(\bar{z}+1)]^{n}} \\
\times(1+ & \left.O\left(\frac{1}{|m|}\right)+O\left(\frac{1}{|m|(\bar{z}-1)^{2}}\right)+O\left(\frac{1}{|m|\left(\bar{z}-\bar{z}_{+}\right)^{3 / 2}}\right)+O\left(\frac{1}{|m|\left(\bar{z}-\bar{z}_{-}\right)^{3 / 2}}\right)\right) .
\end{aligned}
$$

Here $\Lambda(\bar{z})$ is given by (77), and $c_{ \pm}$are constants.

\section{Asymptotic solution of the multipoint SPECTRAL PROBlem CALCULATION OF THE CORRECTION IN THE SPECTRAL SERIES}

Let us construct asymptotic solutions of the multipoint spectral problem $p(\bar{z})$. Near the singular points $(51)$ we choose the solutions with vanishing characteristic exponents, and at the singular point $\bar{z}_{4}=\infty$ with the exponent $-n+|m|+1$. By $(91), \Phi_{-}^{W K B}(\bar{z})$ has zero exponents at the singular points (51). Moreover, if the conditions (30) and (31) hold, then, for $|\bar{z}| \gg|m|$, we have the asymptotics

$$
\Phi_{-}^{W K B}(\bar{z})=\frac{c_{-}(\sqrt{a}+1)^{n}}{(2+\sqrt{5-a})^{\left(\tilde{\xi}_{k}^{(1)} /(4 \sqrt{5-a})\right.} 2^{|m|}} \bar{z}^{n-|m|-1}\left(1+O\left(\frac{1}{|m|}\right)+O\left(\frac{|m|}{\bar{z}}\right)\right) .
$$

Therefore the WKB approximation $\Phi_{-}^{W K B}(\bar{z})$ yields the asymptotic solution of the multipoint spectral problem in the domains I-IV (see Figure 1).

Now we find the asymptotic solutions near the turning points $\bar{z}_{ \pm}$. It follows from (76) that, near $\bar{z}_{ \pm}$,

$$
\hbar^{2} \frac{d^{2} Y}{d \bar{z}^{2}}-\left(\tau_{ \pm}^{2}\left(\bar{z}-\bar{z}_{ \pm}\right)+O\left(\left(\bar{z}-\bar{z}_{ \pm}\right)^{2}\right)+O\left(\frac{1}{|m|}\right)\right) Y=0
$$

Therefore, the principal terms of the asymptotic expansions near $\bar{z}_{ \pm}$can be expressed via Airy functions:

$$
y_{ \pm}^{0}=\alpha_{1, \pm} \operatorname{Ai}\left(|m|^{2 / 3} \tau_{ \pm}^{2 / 3}\left(\bar{z}-\bar{z}_{ \pm}\right)\right)+\alpha_{2, \pm} \operatorname{Bi}\left(|m|^{2 / 3} \tau_{ \pm}^{2 / 3}\left(\bar{z}-\bar{z}_{ \pm}\right)\right) .
$$

Here $\alpha_{1, \pm}, \alpha_{2, \pm}$ are constants, and $\tau_{ \pm}$is given by (90).

Let us construct the solution of the multipoint spectral problem near the turning point $\bar{z}=1$ and also determine the numbers $\tilde{\xi}_{k}^{(1)}$. In the equation (76), we expand the functions 
$Q_{0}(\bar{z})$ and $R(\bar{z})$ into their Taylor series in a neighborhood of $\bar{z}=1$. As a result, after the substitution

$$
\bar{u}=\frac{\sqrt{|m|}(\bar{z}-1)}{\beta},
$$

where

$$
\beta=\frac{\sqrt{5}}{\sqrt[4]{5-a}}
$$

we have

(96) $\frac{d^{2} Y}{d \bar{u}^{2}}+\left\{-\frac{\bar{u}^{2}}{4}+v+\frac{1}{2}+\frac{1}{\sqrt{|m|}}\left(\frac{3}{4} \beta \bar{u}^{3}-\frac{\tilde{\xi}_{k}^{(1)} \beta^{3} \bar{u}}{10}\right)+O\left(\frac{1}{|m|}\right)+O\left(\frac{\bar{u}^{4}}{|m|}\right)\right\} Y=0$.

Here

$$
v=\frac{\tilde{\xi}_{k}^{(1)} \beta^{2}}{20}-\frac{1}{2} .
$$

We want to find the asymptotic solution of (96) in the form

$$
Y=y_{0}(\bar{u})+\frac{y_{1}(\bar{u})}{\sqrt{|m|}}+O\left(\frac{y_{0}}{|m|}\right)+O\left(\frac{\bar{u}^{6} y_{0}}{|m|}\right)+O\left(\frac{\bar{u}}{|m|} \frac{d y_{0}}{d \bar{u}}\right)+O\left(\frac{\bar{u}^{3}}{|m|} \frac{d y_{0}}{d \bar{u}}\right) .
$$

Then the principal term of the asymptotics satisfies the Weber differential equation

$$
\frac{d^{2} y_{0}}{d \bar{u}^{2}}+\left(-\frac{\bar{u}^{2}}{4}+v+\frac{1}{2}\right) y_{0}=0
$$

whose general solution can be represented as a linear combination of parabolic cylinder functions

$$
y_{0}=\alpha_{1} D_{v}(\bar{u})+\alpha_{2} D_{-v-1}(i \bar{u}) .
$$

Here $\alpha_{1}$ and $\alpha_{2}$ are constants.

Let us match the asymptotic expansions. To this end, we utilize the asymptotic expansions of parabolic cylinder functions 22. As $|z| \rightarrow \infty$, we have

$$
\begin{gathered}
D_{v}(z)=z^{v} e^{-z^{2} / 4}\left(1+O\left(z^{-2}\right)\right), \quad|\arg z|<\frac{3 \pi}{4} \\
D_{v}(z)=z^{v} e^{-z^{2} / 4}\left(1+O\left(z^{-2}\right)\right)-\frac{\sqrt{2 \pi}}{\Gamma(-v)} e^{v \pi i} z^{-v-1} e^{z^{2} / 4}\left(1+O\left(z^{-2}\right)\right), \\
\frac{\pi}{4}<\arg z<\frac{5 \pi}{4},
\end{gathered}
$$

where $\Gamma(v)$ is the gamma function. Therefore, when $|\bar{u}| \rightarrow \infty,|\arg \bar{u}|<\pi / 4$,

$$
\begin{aligned}
y_{0} & =\alpha_{1}\left(\frac{\sqrt{|m|}(\bar{z}-1)}{\beta}\right)^{v} \exp \left(-\frac{|m| \sqrt{5-a}(\bar{z}-1)^{2}}{20}\right)\left(1+O\left(\frac{1}{|m|(\bar{z}-1)^{2}}\right)\right) \\
& +\alpha_{2}\left(\frac{i \sqrt{|m|}(\bar{z}-1)}{\beta}\right)^{-v-1} \exp \left(\frac{|m| \sqrt{5-a}(\bar{z}-1)^{2}}{20}\right)\left(1+O\left(\frac{1}{|m|(\bar{z}-1)^{2}}\right)\right) .
\end{aligned}
$$

The first summand in (102) decreases exponentially, whereas the second increases exponentially. In the domain IV near $\bar{z}=1$ (at a distance of order $|m|^{-3 / 8}$ ), when $|\arg \bar{u}|<\pi / 4$, the function $y_{0}$ must match $Y_{-}^{W K B}$, whose expansion is (89). Since the terms of that expansion decrease exponentially when $|\arg \bar{u}|<\pi / 4$, the constant

$$
\alpha_{2}=0 \text {. }
$$


Furthermore, by (101), when $|\bar{u}| \rightarrow \infty, 3 \pi / 4<\arg \bar{u}<5 \pi / 4$,

$$
\begin{aligned}
y_{0} & =\alpha_{1}\left[\left(\frac{\sqrt{|m|}(\bar{z}-1)}{\beta}\right)^{v} \exp \left(-\frac{|m| \sqrt{5-a}(\bar{z}-1)^{2}}{20}\right)\left(1+O\left(\frac{1}{|m|(\bar{z}-1)^{2}}\right)\right)\right. \\
& \left.-\frac{\sqrt{2 \pi} \exp (v \pi i)}{\Gamma(-v)}\left(\frac{\sqrt{|m|}(\bar{z}-1)}{\beta}\right)^{-v-1} \exp \left(\frac{|m| \sqrt{5-a}(\bar{z}-1)^{2}}{20}\right)\left(1+O\left(\frac{1}{|m|(\bar{z}-1)^{2}}\right)\right)\right] .
\end{aligned}
$$

Here, the first summand decreases exponentially, whereas the second increases exponentially. In the domain I near $\bar{z}=1$, when $3 \pi / 4<\arg \bar{u}<5 \pi / 4$, the function $y_{0}$ must also match $Y_{-}^{W K B}$, whose expansion is (89). Since the terms of that expansion decrease exponentially when $3 \pi / 4<\arg \bar{u}<5 \pi / 4$, we obtain a condition

$$
\frac{1}{\Gamma(-v)}=0
$$

As is known [32, the gamma function $\Gamma(-v)$ has poles only when

$$
v=k, \quad k=0,1,2, \ldots .
$$

Since $v$ and $\tilde{\xi}_{k}^{(1)}$ are related by (97), the correction in the spectral series has been determined. Whence

Lemma 7. The numbers $\tilde{\xi}_{k}^{(1)}$ in (75) have the form

$$
\tilde{\xi}_{k}^{(1)}=4 \sqrt{5-a}\left(k+\frac{1}{2}\right), \quad k=0,1,2, \ldots
$$

It follows from (99), (103)-(105) that

$$
y_{0}=\alpha_{1} D_{k}(\bar{u}), \quad k=0,1,2, \ldots,
$$

where

$$
\alpha_{1}=\frac{c_{-}^{(1)} \beta^{k}}{|m|^{k / 2}}=\frac{c_{-} 5^{(k+1) / 2}}{|m|^{k / 2}(\sqrt{5-a})^{3 k / 2+1} 4^{k+1 / 2}}\left(\frac{(\sqrt{5-a}+2 \sqrt{a})^{\sqrt{a}}}{(\sqrt{5-a}+2) 5^{\sqrt{a} / 2}}\right)^{|m|} .
$$

The functions in the right-hand side of (106) can be expressed via the Hermite polynomials:

$$
y_{0}=\frac{\alpha_{1}}{2^{k / 2}} H_{k}\left(\frac{\bar{u}}{\sqrt{2}}\right) e^{-\bar{u}^{2} / 4}, \quad k=0,1,2, \ldots .
$$

Let us find the next term in the expansion (98). It follows from (96), (97), and (104)-(106) that $y_{1}$ satisfies the equation

$$
\frac{d^{2} y_{1}}{d \bar{u}^{2}}+\left(-\frac{\bar{u}^{2}}{4}+k+\frac{1}{2}\right) y_{1}=\left(-\frac{3}{4} \bar{u}^{3}+2\left(k+\frac{1}{2}\right) \bar{u}\right) \beta \alpha_{1} D_{k}(\bar{u}) .
$$

By direct differentiation, one can prove that the general solution of (109) has the form

$$
y_{1}=-\frac{\beta \alpha_{1}}{2}\left[\bar{u}^{2} D_{k}^{\prime}(\bar{u})-\bar{u} D_{k}(\bar{u})\right]+\alpha_{1,1} D_{k}(\bar{u})+\alpha_{1,2} D_{-k-1}(i \bar{u}) .
$$

Here $\alpha_{1,1}, \alpha_{1,2}$ are constants. The matching condition on $y_{1}$ and the WKB approximation implies that $\alpha_{1,2}=0$. Also, let us set $\alpha_{1,1}=0$, assuming that $\alpha_{1}$ contains a correction of order $|m|^{-1 / 2}$. The function $y_{1}$ can also be expressed via the Hermite polynomials:

$$
y_{1}=\frac{\beta \alpha_{1}}{2^{(k+1) / 2}}\left\{\left[\left(\frac{\bar{u}}{\sqrt{2}}\right)^{3}+\frac{\bar{u}}{\sqrt{2}}\right] H_{k}\left(\frac{\bar{u}}{\sqrt{2}}\right)-\left(\frac{\bar{u}}{\sqrt{2}}\right)^{2} H_{k}^{\prime}\left(\frac{\bar{u}}{\sqrt{2}}\right)\right\} e^{-\bar{u}^{2} / 4} .
$$

Since (67) holds, to find the asymptotic solutions of the multipoint spectral problem near $\bar{z}=1$ it remains to expand the function $E(\bar{z})$ by the powers of $\bar{z}-1$. After the substitution (94), we have 


$$
\begin{aligned}
E(\bar{z})=5^{|m| \sqrt{a} / 2-1 / 2} & \exp \left(\frac{\sqrt{|m|}(\sqrt{a}-1) \beta \bar{u}}{2}+\frac{(5-3 \sqrt{a}) \beta^{2} \bar{u}^{2}}{20}\right) \\
& \times\left\{1+\frac{\beta}{\sqrt{|m|}}\left[\frac{1}{3}\left(\frac{\sqrt{a}}{5}-\frac{1}{2}\right) \beta^{2} \bar{u}^{3}-\bar{u}\right]+O\left(\frac{1}{|m|}\right)+O\left(\frac{\bar{u}^{4}}{|m|}\right)\right\} .
\end{aligned}
$$

Next, we multiply (111) and (98). Since $y_{0}, y_{1}$ are defined by (108), (110), we have an expansion

$$
\begin{aligned}
& p(\bar{z})=p_{0}(\bar{u})+\frac{p_{1}(\bar{u})}{\sqrt{|m|}}+O\left(\frac{p_{0}}{|m|}\right)+O\left(\frac{\bar{u}^{6} p_{0}}{|m|}\right) \\
& \quad+\exp \left(\frac{\sqrt{|m|}(\sqrt{a}-1) \beta \bar{u}}{2}+\frac{(5-3 \sqrt{a}) \beta^{2} \bar{u}^{2}}{20}\right)\left(O\left(\frac{\bar{u}}{|m|} \frac{d y_{0}}{d \bar{u}}\right)+O\left(\frac{\bar{u}^{5}}{|m|} \frac{d y_{0}}{d \bar{u}}\right)\right),
\end{aligned}
$$

where

$$
\begin{aligned}
p_{0}= & \alpha_{1} \mu \exp \left(\frac{\sqrt{|m|}(\sqrt{a}-1) \beta \bar{u}}{2}+\left(-1+\frac{(5-3 \sqrt{a})}{\sqrt{5-a}}\right) \frac{\bar{u}^{2}}{4}\right) H_{k}\left(\frac{\bar{u}}{\sqrt{2}}\right), \\
p_{1}= & \frac{\beta \alpha_{1} \mu}{\sqrt{2}} \exp \left(\frac{\sqrt{|m|}(\sqrt{a}-1) \beta \bar{u}}{2}+\left(-1+\frac{(5-3 \sqrt{a})}{\sqrt{5-a}}\right) \frac{\bar{u}^{2}}{4}\right) \\
& \times\left\{\left[\left(\frac{2(2 \sqrt{a}-5)}{3 \sqrt{5-a}}+1\right)\left(\frac{\bar{u}}{\sqrt{2}}\right)^{3}-\frac{\bar{u}}{\sqrt{2}}\right] H_{k}\left(\frac{\bar{u}}{\sqrt{2}}\right)-\left(\frac{\bar{u}}{\sqrt{2}}\right)^{2} H_{k}^{\prime}\left(\frac{\bar{u}}{\sqrt{2}}\right)\right\} .
\end{aligned}
$$

Here

$$
\mu=5^{|m| \sqrt{a} / 2-1 / 2} 2^{-k / 2} .
$$

This proves

Lemma 8. The asymptotic solution of the multipoint spectral problem near the turning point $\bar{z}=1$ is of the form (112).

We continue the process of matching the asymptotics. By (89) and (100), in the domain $\mathrm{V}$ near $\bar{z}=1$ the function (112) matches $\Phi_{-}^{W K B}(\bar{z})$. Therefore, the WKB approximation equals $\Phi_{-}^{W K B}(\bar{z})$ in this domain, too.

Also, by virtue of the known asymptotics for the Airy functions [32,

$$
\operatorname{Ai}(z)=\frac{1}{2 \sqrt{\pi} \sqrt[4]{z}} \exp \left(-\frac{2}{3} z^{3 / 2}\right)\left(1+O\left(\frac{1}{z^{2}}\right)\right), \quad|\arg z|<\pi,|z| \rightarrow \infty
$$

and

$$
\operatorname{Bi}(z)=\frac{1}{\sqrt{\pi} \sqrt[4]{z}} \exp \left(\frac{2}{3} z^{3 / 2}\right)\left(1+O\left(\frac{1}{z^{2}}\right)\right), \quad|\arg z|<\frac{\pi}{3},|z| \rightarrow \infty,
$$

when $\left|\arg \left(\tau_{ \pm}^{2 / 3}\left(\bar{z}-\bar{z}_{ \pm}\right)\right)\right|<\pi / 3,|m|^{2 / 3}\left(\bar{z}-\bar{z}_{ \pm}\right) \rightarrow \infty$,

$$
\begin{aligned}
y_{ \pm}^{0}= & \frac{\alpha_{1, \pm}}{|m|^{1 / 6} 2 \sqrt{\pi} \tau_{ \pm}^{1 / 6} \sqrt[4]{\bar{z}-\bar{z}_{ \pm}}} \exp \left(-\frac{2|m| \tau_{ \pm}\left(\bar{z}-\bar{z}_{ \pm}\right)^{3 / 2}}{3}\right)\left(1+O\left(\frac{1}{|m|\left(\bar{z}-\bar{z}_{+}\right)^{3 / 2}}\right)\right) \\
& +\frac{\alpha_{2, \pm}}{|m|^{1 / 6} \sqrt{\pi} \tau_{ \pm}^{1 / 6} \sqrt[4]{\bar{z}-\bar{z}_{ \pm}}} \exp \left(\frac{2|m| \tau_{ \pm}\left(\bar{z}-\bar{z}_{ \pm}\right)^{3 / 2}}{3}\right)\left(1+O\left(\frac{1}{|m|\left(\bar{z}-\bar{z}_{+}\right)^{3 / 2}}\right)\right) .
\end{aligned}
$$

In (114), the first summand decreases exponentially, whereas the second increases exponentially. Let us match the expansion (114) near $\bar{z}_{ \pm}$(at a distance of order $|m|^{-1 / 2}$ ) and the WKB approximation $Y_{-}^{W K B}(\bar{z})$, which holds in the domain $\mathrm{V}$ (see Figure 1). By 
(87) and (88), $\Phi_{-}^{W K B}(\bar{z})$ has an exponentially decreasing asymptotics as $\bar{z}$ tends to $\bar{z}_{+}$ and $\bar{z}_{-}$within this domain. Therefore, in the formula (93), $\alpha_{2, \pm}=0$ and

$$
\alpha_{1, \pm}=c_{-}^{( \pm)} 2 \sqrt{\pi} \tau_{ \pm}^{1 / 6}|m|^{1 / 6} .
$$

The asymptotic expansions constructed near $\bar{z}_{+}$and $\bar{z}_{-}$in the domains II and III also $\operatorname{match} Y_{-}^{W K B}(\bar{z})$.

Set

$$
\mu_{1, \pm}=\left(\frac{3}{\sqrt{a}}-1\right) \bar{z}_{\mp}+\frac{2}{\sqrt{a}}, \quad \mu_{2, \pm}=\left(\frac{15}{a^{3 / 2}}-\frac{7}{a^{1 / 2}}-3+a\right) \bar{z}_{\mp}+\frac{5}{a^{3 / 2}}-1 .
$$

Expanding the function (68) into its Taylor series in a neighborhood of $\bar{z}_{+}$and $\bar{z}_{-}$, we have

Lemma 9. The asymptotic solution of the multipoint spectral problem near $\bar{z}_{ \pm}$is of the form

$$
\begin{array}{r}
p(\bar{z})=E\left(\bar{z}_{ \pm}\right) \exp \left(\mu_{1, \pm}|m| \frac{\bar{z}-\bar{z}_{ \pm}}{2}+\mu_{2, \pm}|m| \frac{\left(\bar{z}-\bar{z}_{ \pm}\right)^{2}}{4}\right)\left(y_{ \pm}^{0}+O\left(\left(\bar{z}-\bar{z}_{ \pm}\right) y_{ \pm}^{0}\right)\right. \\
\left.+O\left(|m|\left(\bar{z}-\bar{z}_{ \pm}\right)^{3} y_{ \pm}^{0}\right)+O\left(\frac{1}{|m|} \frac{d y_{ \pm}^{0}}{d \bar{z}}\right)+O\left(\left(\bar{z}-\bar{z}_{ \pm}\right)^{2} \frac{d y_{ \pm}^{0}}{d \bar{z}}\right)\right)
\end{array}
$$

where $y_{ \pm}^{0}=\alpha_{1, \pm} \operatorname{Ai}\left(|m|^{2 / 3} \tau_{ \pm}^{2 / 3}\left(\bar{z}-\bar{z}_{ \pm}\right)\right)$, and the constant $\alpha_{1, \pm}$ is given by (115).

The formula (105) for $\tilde{\xi}_{k}^{(1)}$ allows us to represent the expression (91) for $\Phi_{-}^{W K B}(\bar{z})$ in a final form:

$$
\begin{array}{r}
\Phi_{-}^{W K B}(\bar{z})=\Phi_{-, 0}^{W K B}(\bar{z})\left(1+O\left(\frac{1}{|m|}\right)+O\left(\frac{1}{|m|(\bar{z}-1)^{2}}\right) O\left(\frac{1}{|m|\left(\bar{z}-\bar{z}_{+}\right)^{3 / 2}}\right)+\right. \\
\left.+O\left(\frac{1}{|m|\left(\bar{z}-\bar{z}_{-}\right)^{3 / 2}}\right)\right)
\end{array}
$$

where

$$
\Phi_{-, 0}^{W K B}(\bar{z})=\frac{c_{-}(\bar{z}-1)^{k}[\sqrt{\Lambda(\bar{z})}+\sqrt{a}(\bar{z}+1)]^{n}}{\sqrt[4]{\Lambda(\bar{z})}[2 \sqrt{\Lambda(\bar{z})}+\sqrt{5-a}(\bar{z}+1)]^{k+1 / 2}[\sqrt{\Lambda(\bar{z})}+\bar{z}+1]^{|m|}},
$$

$k=0,1,2, \ldots, c_{-}$is a constant, and $\Lambda(\bar{z})$ is determined by $(77)$.

Thus, the asymptotic solution of the multipoint spectral problem has been constructed. The numbers $\tilde{\xi}_{k}$ are given by (75) and (105), and the antiholomorphic function $p(\bar{z})$ is obtained by matching the asymptotics. More precisely, $p(\bar{z})=\Phi_{-}^{W K B}(\bar{z})$ is the asymptotic solution of the equation (48) on the entire complex plane with the exception of small neighborhoods of the turning points $\bar{z}_{+}$and $\bar{z}_{-}, 1$, and the arc $\smile \bar{z}_{-}, \bar{z}_{+}$. Near the points $\bar{z}_{+}, \bar{z}_{-}$and 1 , the function $p(\bar{z})$ is given by the formulas (116) and (112), respectively. The asymptotics match at distances of order $|m|^{-1 / 2}$ from the points $\bar{z}_{ \pm}$and of order $|m|^{-3 / 8}$ from the point 1 .

Finally, near the arc $\smile \bar{z}_{-}, \bar{z}_{+}$, which is a Stokes line, the desired asymptotics can be written as the sum of two functions $\Phi_{-}^{W K B}(\bar{z})$, where in the first function we take the branch corresponding to the counterclockwise direction around the point $\bar{z}_{ \pm}$, and in the second to the clockwise direction. Such a representation for $p(\bar{z})$ follows from the known expansion of the Airy functions [32]:

$$
\operatorname{Ai}(-z)=\frac{1}{\sqrt{\pi} \sqrt[4]{z}} \sin \left(\frac{2}{3} z^{3 / 2}+\frac{\pi}{4}\right)+O\left(\frac{1}{z^{7 / 4}}\right), \quad|\arg z|<\frac{2 \pi}{3},|z| \rightarrow \infty .
$$




\section{Asymptotics of the polynomials $\Phi(\bar{z})$. The Discrete WKB method}

Let us substitute the asymptotic solution of the multipoint spectral problem $p(\bar{z})$ in the right-hand side of (57) and compute the asymptotics of the resulting integral. The integrand in (57) has no saddle points. Therefore we can use the Cauchy integral theorem.

Lemma 10. If $\bar{z} \notin \smile \bar{z}_{-}, \bar{z}_{+}$and

$$
|\bar{z}-1| \gtrsim|m|^{-3 / 8}, \quad\left|\bar{z}-\bar{z}_{ \pm}\right| \gtrsim|m|^{-1 / 2},
$$

then the polynomial $\Phi(\bar{z})$ determined by $(57)$ can be represented as

$$
\Phi(\bar{z})=\Phi_{-}^{W K B}(\bar{z})+N(\bar{z}),
$$

where

$$
N(\bar{z})=-\frac{\bar{z}^{n-|m|}}{2 \pi i} \oint_{\gamma_{+,-}} \frac{\Phi_{-}^{W K B}(\bar{u}) d \bar{u}}{\bar{u}^{n-|m|}(\bar{u}-\bar{z})} .
$$

Here the closed contour $\gamma_{+,-}$is the arc $\cup \bar{z}_{-}, \bar{z}_{+}$of the circle, traversed twice along the edges of the cut joining $\bar{z}_{-}$and $\bar{z}_{+}$. It is oriented counterclockwise.

Proof. Suppose $\bar{z}$ lies outside the cycle $\gamma, \bar{z} \notin \smile \bar{z}_{-}, \bar{z}_{+}$and the conditions (119) hold. Consider the contours shown in Figure 2. The circle centered at $\bar{z}=1$ has radius of order $|m|^{-3 / 8}$. For the circles centered at $\bar{z}_{ \pm}$the order of the radii is $|m|^{-1 / 2}$. Using the Cauchy integral theorem and the Cauchy integral formula, we rewrite the integral over $\gamma$ in the following form:

$$
\begin{aligned}
& \text { 122) } \Phi(\bar{z})=\frac{\bar{z}^{n-|m|}}{2 \pi i} \oint_{\gamma} \frac{\Phi_{-}^{W K B}(\bar{u}) d \bar{u}}{\bar{u}^{n-|m|}(\bar{u}-\bar{z})}=-\frac{\bar{z}^{n-|m|}}{2 \pi i} \oint_{\gamma_{\bar{z}}} \frac{\Phi_{-}^{W K B}(\bar{u}) d \bar{u}}{\bar{u}^{n-|m|}(\bar{u}-\bar{z})} \\
& -\frac{\bar{z}^{n-|m|}}{2 \pi i} \oint_{\gamma_{1}} \frac{\Phi_{-}^{W K B}(\bar{u}) d \bar{u}}{\bar{u}^{n-|m|}(\bar{u}-\bar{z})}-\frac{\bar{z}^{n-|m|}}{2 \pi i} \oint_{\tilde{\gamma}} \frac{\Phi_{-}^{W K B}(\bar{u}) d \bar{u}}{\bar{u}^{n-|m|}(\bar{u}-\bar{z})}-\frac{\bar{z}^{n-|m|}}{2 \pi i} \oint_{\gamma_{\infty}} \frac{\Phi_{-}^{W K B}(\bar{u}) d \bar{u}}{\bar{u}^{n-|m|}(\bar{u}-\bar{z})} .
\end{aligned}
$$

Let us examine the summands in (122). It follows from the Cauchy integral formula that

$$
-\frac{\bar{z}^{n-|m|}}{2 \pi i} \oint_{\gamma_{\bar{z}}} \frac{\Phi_{-}^{W K B}(\bar{u}) d \bar{u}}{\bar{u}^{n-|m|}(\bar{u}-\bar{z})}=\Phi_{-}^{W K B}(\bar{z}) .
$$

Furthermore, since the integrand is antiholomorphic,

$$
\oint_{\gamma_{1}} \frac{\Phi_{-}^{W K B}(\bar{u}) d \bar{u}}{\bar{u}^{n-|m|}(\bar{u}-\bar{z})}=0
$$

and

$$
\oint_{\gamma_{\infty}} \frac{\Phi_{-}^{W K B}(\bar{u}) d \bar{u}}{\bar{u}^{n-|m|}(\bar{u}-\bar{z})}=0
$$

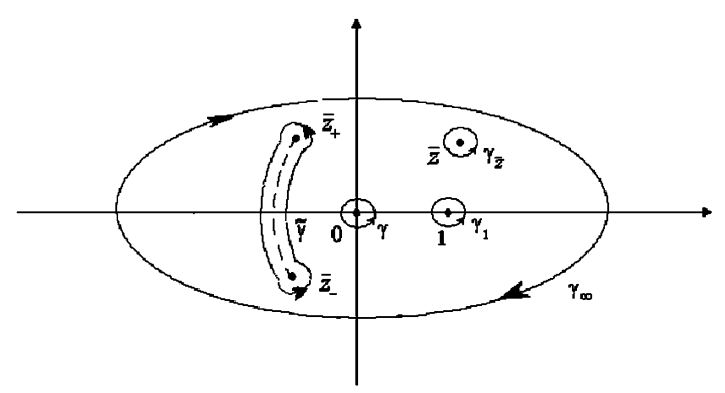

Figure 2 
by the residue theorem and (92). Finally, taking into account (87) and (88), deform $\gamma$ into $\gamma_{+,-}$. As a result, (122) rewrites as (120).

Suppose now that $\bar{z}$ lies inside $\gamma$. Then

$$
\Phi(\bar{z})=\Phi_{-}^{W K B}(\bar{z})+\frac{\bar{z}^{n-|m|}}{2 \pi i} \oint_{\gamma} \frac{\Phi_{-}^{W K B}(\bar{u}) d \bar{u}}{\bar{u}^{n-|m|}(\bar{u}-\bar{z})} .
$$

The integral in (123) rewrites similarly to the case when $\bar{z}$ was outside $\gamma$. Therefore, we again obtain (120).

Now suppose $\bar{z}$ lies inside $\gamma_{1}$. Similar to (122), we have

$$
\Phi(\bar{z})=-\frac{\bar{z}^{n-|m|}}{2 \pi i} \oint_{\gamma_{1}} \frac{p(\bar{u}) d \bar{u}}{\bar{u}^{n-|m|}(\bar{u}-\bar{z})}-\frac{\bar{z}^{n-|m|}}{2 \pi i} \oint_{\gamma_{\infty}} \frac{\Phi_{-}^{W K B}(\bar{u}) d \bar{u}}{\bar{u}^{n-|m|}(\bar{u}-\bar{z})}-\frac{\bar{z}^{n-|m|}}{2 \pi i} \oint_{\tilde{\gamma}} \frac{\Phi_{-}^{W K B}(\bar{u}) d \bar{u}}{\bar{u}^{n-|m|}(\bar{u}-\bar{z})} .
$$

The integrals over $\gamma_{\infty}$ and $\tilde{\gamma}$ in (124) were considered in the proof of Lemma 10, and the integral over $\gamma_{1}$ can be computed using the Cauchy integral formula. Thus,

$$
\Phi(\bar{z})=p(\bar{z})+N(\bar{z}),
$$

where $p(\bar{z})$ and $N(\bar{z})$ are determined by (112) and (121).

Let us estimate the integral in (121):

$$
|N(\bar{z})| \leq \frac{|\bar{z}|^{n-|m|}}{2 \pi} \oint_{\gamma_{+,-}} \frac{\left|\Phi_{-}^{W K B}(\bar{u})\right||d \bar{u}|}{|\bar{u}-\bar{z}|}
$$

Since $\smile \bar{z}_{-}, \bar{z}_{+}$is a Stokes line and because of (77), (78), and (80), we have that, on this arc,

$$
\frac{|\sqrt{\Lambda(\bar{u})}+\sqrt{a}(\bar{u}+1)|^{n}}{|\sqrt{\Lambda(\bar{u})}+\bar{u}+1|^{|m|}\left|\bar{u}^{2}+3 \bar{u}+1\right|^{n / 2}}=\frac{\left(\sqrt{a}\left|\bar{z}_{ \pm}+1\right|\right)^{n}}{\left|\bar{z}_{ \pm}+1\right|^{|m|} a^{n / 2}}=(\sqrt{a-1})^{(\sqrt{a}-1)|m|}
$$

Furthermore, it follows from (68) and (127) that

$$
\begin{array}{r}
\left|\Phi_{-}^{W K B}(\bar{u})\right|=\frac{\left|c_{-}\right|\left|\bar{u}^{2}+3 \bar{u}+1\right|^{\sqrt{a}|m| / 2}(\sqrt{a-1})^{(\sqrt{a}-1)|m|} \vartheta(\bar{u})}{\sqrt[4]{|\Lambda(\bar{u})|}}\left(1+O\left(\frac{1}{|m|}\right)\right. \\
\left.+O\left(\frac{1}{|m|\left|\bar{u}-\bar{z}_{+}\right|^{3 / 2}}\right)+O\left(\frac{1}{|m|\left|\bar{u}-\bar{z}_{-}\right|^{3 / 2}}\right)\right) .
\end{array}
$$

Here

$$
\vartheta(\bar{u})=\frac{|\bar{u}-1|^{k}}{|2 \sqrt{\Lambda(\bar{u})}+\sqrt{5-a}(\bar{u}+1)|^{k+1 / 2}}
$$

is a continuous function, and $\bar{u} \in \smile \bar{z}_{-}, \bar{z}_{+}$. For $\bar{u}=e^{i \varphi} \in \smile \bar{z}_{-}, \bar{z}_{+}$, we have

$$
\left|\bar{u}^{2}+3 \bar{u}+1\right|=|2 \cos \varphi+3|,
$$

and therefore $\max _{\bar{u} \in \cup} \bar{z}_{-} \bar{z}_{+}\left|\bar{u}^{2}+3 \bar{u}+1\right|=a$ is attained at $\bar{u}=\bar{z}_{ \pm}$. Hence, the largest contribution to the asymptotics of the integral (126) comes from small neighborhoods of the turning points $\bar{z}_{ \pm}$. Since 33 .

$$
\int_{0}^{\infty} x^{-1 / 4} e^{-|m| x} d x=|m|^{-3 / 4} \Gamma\left(\frac{3}{4}\right)
$$

we have

Lemma 11. If $|m| \rightarrow \infty$ and $\bar{z} \notin \smile \bar{z}_{-}, \bar{z}_{+}$, then

$$
|N(\bar{z})| \leq T|m|^{-3 / 4}\left|c_{-}\right|\left(a^{\sqrt{a}}(a-1)^{\sqrt{a}-1}\right)^{|m| / 2}|\bar{z}|^{n-|m|}\left(\frac{1}{\left|\bar{z}-\bar{z}_{+}\right|}+\frac{1}{\left|\bar{z}-\bar{z}_{-}\right|}\right) .
$$

Here $T$ is a constant. 
Now we use the fact that, on the circle $|\bar{z}|=1$, the function

$$
M(\bar{z})=\frac{|\sqrt{\Lambda(\bar{z})}+\sqrt{a}(\bar{z}+1)|^{\sqrt{a}}}{|\sqrt{\Lambda(\bar{z})}+\bar{z}+1|}
$$

attains a maximum value at $\bar{z}=1$. Indeed, by (127) and (128), on the arc $\smile \bar{z}_{-}, \bar{z}_{+}$, $M(\bar{z})$ has maximum values at the turning points $\bar{z}_{ \pm}$. On the remaining part of the circle $|\bar{z}|=1$, we have

$$
M\left(e^{i \varphi}\right)=\frac{\left(\sqrt{4 \cos ^{2} \frac{\varphi}{2}+1-a}+2 \sqrt{a} \cos \frac{\varphi}{2}\right)^{\sqrt{a}}}{\sqrt{4 \cos ^{2} \frac{\varphi}{2}+1-a}+2 \cos \frac{\varphi}{2}} .
$$

Since

$$
\frac{d}{d \varphi} M\left(e^{i \varphi}\right)=-\frac{(a-1) M\left(e^{i \varphi}\right) \sin \frac{\varphi}{2}}{\sqrt{4 \cos ^{2} \frac{\varphi}{2}+1-a}+2 \sqrt{a} \cos \frac{\varphi}{2}},
$$

the sign of the derivative shows that $M(\bar{z})$ increases on $\smile \bar{z}_{+}, 1$ as the argument approaches $\bar{z}=1$, whereas on $\smile 1, \bar{z}_{-}$it decreases. Therefore, the only maximum point of $M(\bar{z})$ on the circle $|\bar{z}|=1$ is $\bar{z}=1$.

Since

$$
M(1)>M\left(\bar{z}_{ \pm}\right)=\sqrt{a}^{\sqrt{a}} \sqrt{a-1}^{\sqrt{a}-1},
$$

it follows from (129) and (118) that in a neighborhood of $\bar{z}=1$, which provides the main contribution to the computation of the averages, $N(\bar{z})$ is exponentially small compared to $p(\bar{z})$. Here $p(\bar{z})$ is the expansion (112), which is valid near $\bar{z}=1$ and matches $\Phi_{-}^{W K B}(\bar{z})$. Notice that near $\bar{z}=1$ the derivatives of $N(\bar{z})$ of bounded (as $|m| \rightarrow \infty$ ) order will also be exponentially small.

Now we want to show that $N(\bar{z})$ is small compared with $\Phi_{-}^{W K B}(\bar{z})$ for $|\bar{z}| \gg|m|$ as well. The estimate (129) when $|\bar{z}| \rightarrow \infty$ is too rough. Indeed, the fact that $N(\bar{z})$ is small follows from (129) and (92) only when $a \in\left(1, a_{*}\right)$, where $a_{*} \approx 2.34$, because only for these values of the parameter $a$ does the inequality

$$
\frac{(\sqrt{a}+1)^{\sqrt{a}}}{2}>\sqrt{a}^{\sqrt{a}} \sqrt{a-1}^{\sqrt{a}-1}
$$

hold. Let us find an estimate on $N(\bar{z})$ valid for all values $a \in(1,5)$.

By (117) and (121), as $|\bar{z}| \rightarrow \infty$,

$$
N(\bar{z})=\frac{\bar{z}^{n-|m|-1}}{2 \pi i} \oint_{\gamma_{+,-}} \frac{\Phi_{-, 0}^{W K B}(\bar{u})}{\bar{u}^{n-|m|}} d \bar{u}\left(1+O\left(\frac{1}{|m|}\right)+O\left(\frac{1}{\bar{z}}\right)\right) .
$$

The integrand in (131) has no saddle points. To find the asymptotics of the integral, we express it in terms of the coefficients of a power series converging near zero,

$$
\Phi_{-, 0}^{W K B}(\bar{z})=\sum_{j=0}^{\infty} \zeta_{j} \bar{z}^{j}
$$

and then use the discrete WKB method [34, 35.

It follows from (118) that $\Phi_{-, 0}^{W K B}(\bar{z})$ satisfies the relation

$$
\Phi_{-, 0}^{W K B}\left(\frac{1}{\bar{z}}\right)=(-1)^{k} \frac{\Phi_{-, 0}^{W K B}(\bar{z})}{\bar{z}^{n-|m|-1}} .
$$


Therefore, as $|\bar{z}| \rightarrow \infty$, we have an expansion

$$
\Phi_{-, 0}^{W K B}(\bar{z})=(-1)^{k} \sum_{j=0}^{\infty} \zeta_{j} \bar{z}^{n-|m|-1-j}
$$

Furthermore, deforming $\gamma_{+,-}$, we represent the integral in (131) as the sum of two integrals over cycles around $\bar{u}=0$ and $\bar{u}=\infty$. In view of (132) and (133),

$$
\frac{1}{2 \pi i} \oint_{\gamma_{+,-}} \frac{\Phi_{-, 0}^{W K B}(\bar{u})}{\bar{u}^{n-|m|}} d \bar{u}=\zeta_{n-|m|-1}-(-1)^{k} \zeta_{0} .
$$

By (48), the coefficients $\zeta_{j}$ of the power series (132) satisfy a three-term recurrence relation

$$
R\left(\frac{j}{|m|}\right) \zeta_{j+1}+Q\left(\frac{j}{|m|}\right) \zeta_{j}+P\left(\frac{j}{|m|}\right) \zeta_{j-1}=0
$$

Here

$$
\begin{gathered}
R(x)=x^{2}+x+\frac{1}{|m|}(2 x+1)+O\left(\frac{1}{|m|^{2}}\right), \\
P(x)=(x-\sqrt{a})(x-\sqrt{a}+1)+O\left(\frac{1}{|m|^{2}}\right), \\
Q(x)=3 x^{2}-3(\sqrt{a}-1) x+\frac{(\sqrt{a}-1)(\sqrt{a}-5)}{4} \\
+\frac{1}{|m|}\left(3 x-\frac{3(\sqrt{a}-1)}{2}+\sqrt{5-a}\left(k+\frac{1}{2}\right)\right)+O\left(\frac{1}{|m|^{2}}\right) .
\end{gathered}
$$

The asymptotics of $\zeta_{j}, j=0, \ldots, n-|m|-1$, as $|m| \rightarrow \infty$, can be found with the aid of the discrete WKB method. Set

$$
A(x)=\sqrt{\frac{P(x)}{R(x)}}, \quad B(x)=-\frac{Q(x)}{2 \sqrt{P(x) R(x)}} .
$$

By [34], when $B(x)>1$, the principal terms of the WKB approximations $\zeta_{j, \pm}^{(0)}$ are of the form

$$
\begin{aligned}
\zeta_{j, \pm}^{(0)}=\frac{1}{\sqrt[4]{B^{2}(j /|m|)-1}} \exp \left(| m | \int _ { j _ { 0 } / | m | } ^ { j / | m | } \left\{\ln A(x) \pm\left(\ln \left(B(x)+\sqrt{B^{2}(x)-1}\right)\right.\right.\right. \\
\left.\left.\left.-\frac{1}{2 A(x)} \frac{d A(x)}{d x} \frac{B(x)}{\sqrt{B^{2}(x)-1}}\right)\right\} d x\right)
\end{aligned}
$$

Here $j_{0}$ is a constant.

Now make the substitution

$$
x_{1}=x-\frac{\sqrt{a}-1}{2}+\frac{1}{2|m|} .
$$

If $x=j /|m|$, where $j=0, \ldots, n-|m|-1$, then

$$
x_{1} \in\left[-\frac{\sqrt{a}-1}{2}+\frac{1}{2|m|}, \frac{\sqrt{a}-1}{2}-\frac{1}{2|m|}\right] .
$$

From (136)-(139), we have 
Lemma 12. As $|m| \rightarrow \infty$,

$$
\begin{aligned}
& A\left(x_{1}\right)= \sqrt{\frac{\left(x_{1}-(\sqrt{a}+1) / 2\right)\left(x_{1}-(\sqrt{a}-1) / 2\right)}{\left(x_{1}+(\sqrt{a}-1) / 2\right)\left(x_{1}+(\sqrt{a}+1) / 2\right)}}\left\{1-\frac{1}{4|m|}\left[\frac{1}{x_{1}-(\sqrt{a}+1) / 2}\right.\right. \\
&\left.\left.+\frac{1}{x_{1}-(\sqrt{a}-1) / 2}+\frac{1}{x_{1}+(\sqrt{a}-1) / 2}+\frac{1}{x_{1}+(\sqrt{a}+1) / 2}\right]+O\left(\frac{1}{|m|^{2}}\right)\right\}, \\
& B\left(x_{1}\right)= \frac{1}{4 \sqrt{\left(x_{1}^{2}-(\sqrt{a}-1)^{2} / 4\right)\left(x_{1}^{2}-(\sqrt{a}+1)^{2} / 4\right)}}\left\{-6 x_{1}^{2}+a-1\right. \\
&\left.-\frac{1}{|m|}\left[2 \sqrt{5-a}\left(k+\frac{1}{2}\right)+3 \sqrt{a}+\frac{4 \sqrt{a}(a+11) x_{1}^{2}-\sqrt{a}(a-1)^{2}}{16\left(x_{1}^{2}-(\sqrt{a}-1)^{2} / 4\right)\left(x_{1}^{2}-(\sqrt{a}+1)^{2} / 4\right)}\right]+O\left(\frac{1}{|m|^{2}}\right)\right\} .
\end{aligned}
$$

Let us compute the functions in (140):

$$
\begin{aligned}
& \ln A\left(x_{1}\right)=\frac{1}{2} \ln \left(\frac{\left(x_{1}-(\sqrt{a}+1) / 2\right)\left(x_{1}-(\sqrt{a}-1) / 2\right)}{\left(x_{1}+(\sqrt{a}-1) / 2\right)\left(x_{1}+(\sqrt{a}+1) / 2\right)}\right) \\
& -\frac{x_{1}\left(4 x_{1}^{2}-a-1\right)}{4|m|\left(x_{1}^{2}-(\sqrt{a}-1)^{2} / 4\right)\left(x_{1}^{2}-(\sqrt{a}+1)^{2} / 4\right)}+O\left(\frac{1}{|m|^{2}}\right), \\
& \text { (142) } \ln \left(B\left(x_{1}\right)+\sqrt{B^{2}\left(x_{1}\right)-1}\right)=\ln \left(\frac{a-1-6 x_{1}^{2}+2 x_{1} \sqrt{5 x_{1}^{2}+5-a}}{4 \sqrt{\left(x_{1}^{2}-(\sqrt{a}-1)^{2} / 4\right)\left(x_{1}^{2}-(\sqrt{a}+1)^{2} / 4\right)}}\right) \\
& -\frac{1}{2|m| x_{1} \sqrt{5 x_{1}^{2}+5-a}}\left\{2 \sqrt{5-a}\left(k+\frac{1}{2}\right)+3 \sqrt{a}\right. \\
& \left.+\frac{\sqrt{a}\left(4(a+11) x_{1}^{2}-(a-1)^{2}\right)}{16\left(x_{1}^{2}-(\sqrt{a}-1)^{2} / 4\right)\left(x_{1}^{2}-(\sqrt{a}+1)^{2} / 4\right)}\right\}+O\left(\frac{1}{|m|^{2}}\right), \\
& \frac{1}{\sqrt[4]{B^{2}\left(x_{1}\right)-1}}=\frac{\sqrt{2} \sqrt[4]{\left(x_{1}^{2}-(\sqrt{a}-1)^{2} / 4\right)\left(x_{1}^{2}-(\sqrt{a}+1)^{2} / 4\right)}}{\sqrt[4]{x_{1}^{2}\left(5 x_{1}^{2}+5-a\right)}}+O\left(\frac{1}{|m|}\right) \\
& -\frac{1}{2 A\left(x_{1}\right)} \frac{d A\left(x_{1}\right)}{d x_{1}} \frac{B\left(x_{1}\right)}{\sqrt{B^{2}\left(x_{1}\right)-1}} \\
& =-\frac{\sqrt{a}\left(4 x_{1}^{2}-a+1\right)\left(-6 x_{1}^{2}+a-1\right)}{16 x_{1} \sqrt{5 x_{1}^{2}+5-a}\left(x_{1}^{2}-(\sqrt{a}-1)^{2} / 4\right)\left(x_{1}^{2}-(\sqrt{a}+1)^{2} / 4\right)}+O\left(\frac{1}{|m|}\right) .
\end{aligned}
$$

Notice that the formulas (140) of the discrete WKB method are not applicable near a "turning point" where the number

$$
j_{1}=j-(n-|m|-1) / 2
$$

is zero and also near the end points $j=0$ and $j=n-|m|-1$. For such $j$ one needs to construct additional expansions.

Let us construct the asymptotics of $\zeta_{j}$ when $0 \leq j \lesssim|m|^{1 / 3}$. From (118), we have

$$
\begin{gathered}
\zeta_{0}=\frac{c_{-}(-1)^{k}}{(2+\sqrt{5-a})^{k+1 / 2}}\left(\frac{(\sqrt{a}+1)^{\sqrt{a}}}{2}\right)^{|m|}, \\
\zeta_{1}=\left(\frac{|m|(\sqrt{a}-1)(5-\sqrt{a})}{4}+\frac{a-1}{4}-\left(k+\frac{1}{2}\right) \sqrt{5-a}\right) \zeta_{0} .
\end{gathered}
$$

Let

$$
\zeta_{j}=\frac{|m|^{j}}{j !} \varpi_{j}
$$


Then, for $1 \leq j \lesssim|m|^{1 / 3}$, the coefficients $\varpi_{j}$ satisfy the recurrence relation

$$
\begin{aligned}
& \left(-\frac{4 \sqrt{a}(\sqrt{a}-1)(j-1)}{|m|}+O\left(\frac{j^{2}}{|m|^{2}}\right)\right) \varpi_{j-1} \\
& \quad+\left((\sqrt{a}-1)(5-\sqrt{a})+O\left(\frac{j}{|m|}\right)\right) \varpi_{j}+\left(-4+O\left(\frac{j}{|m|}\right)\right) \varpi_{j+1}=0
\end{aligned}
$$

which follows from (135). Since

$$
\varpi_{j+1}=\left(\frac{(\sqrt{a}-1)(5-\sqrt{a})}{4}+O\left(\frac{j}{|m|}\right)\right) \varpi_{j},
$$

we have

Lemma 13. If $1 \leq j \lesssim|m|^{1 / 3}$, then

$$
\zeta_{j}=\frac{|m|^{j}}{j !}\left(\frac{(\sqrt{a}-1)(5-\sqrt{a})}{4}\right)^{j} \zeta_{0}\left(1+O\left(\frac{j^{2}}{|m|}\right)\right) .
$$

By the Stirling formula, when $j$ is of order $|m|^{1 / 3}$,

$$
\zeta_{j}=\frac{1}{\sqrt{2 \pi j}}\left(\frac{|m| e(\sqrt{a}-1)(5-\sqrt{a})}{4 j}\right)^{j} \zeta_{0}\left(1+O\left(\frac{1}{|m|^{1 / 3}}\right)\right) .
$$

Now substitute the expansions (141)-(144) in (140). Then, for $j$ of order $|m|^{1 / 3}$, the right-hand side of $(147)$ matches $c_{1} \zeta_{j,-}^{(0)}$. Here $c_{1}$ is a constant. This yields

Lemma 14. When $|m|^{1 / 3} \lesssim j, j_{1} \lesssim-|m|^{3 / 5}$, we have the asymptotics $\zeta_{j}=c_{1} \zeta_{j,-}^{(0)}$, where

$$
\begin{array}{r}
\zeta_{j,-}^{(0)}=\frac{\sqrt{2} \sqrt[4]{\left(x_{1}^{2}-(\sqrt{a}-1)^{2} / 4\right)\left(x_{1}^{2}-(\sqrt{a}+1)^{2} / 4\right)}}{\sqrt[4]{x_{1}^{2}\left(5 x_{1}^{2}+5-a\right)}} \exp \left\{|m| \int_{x_{1}^{0}}^{j_{1} /|m|} f_{0}\left(x_{1}\right) d x_{1}\right. \\
\left.+\int_{x_{1}^{0}}^{j_{1} /|m|} f_{1}\left(x_{1}\right) d x_{1}+O\left(\frac{1}{|m|^{1 / 5}}\right)\right\}
\end{array}
$$

$$
\begin{gathered}
f_{0}\left(x_{1}\right)=-\ln \left(\frac{a-1-6 x_{1}^{2}+2 x_{1} \sqrt{5 x_{1}^{2}+5-a}}{4\left(x_{1}-(\sqrt{a}+1) / 2\right)\left(x_{1}-(\sqrt{a}-1) / 2\right)}\right) \\
f_{1}\left(x_{1}\right)=-\frac{x_{1}\left(4 x_{1}^{2}-a-1\right)}{4\left(x_{1}^{2}-(\sqrt{a}-1)^{2} / 4\right)\left(x_{1}^{2}-(\sqrt{a}+1)^{2} / 4\right)} \\
+\frac{1}{2 x_{1} \sqrt{5 x_{1}^{2}+5-a}}\left\{2 \sqrt{5-a}\left(k+\frac{1}{2}\right)+3 \sqrt{a}\right. \\
\left.\quad+\frac{\sqrt{a}\left(4(a+11) x_{1}^{2}-(a-1)^{2}\right)}{16\left(x_{1}^{2}-(\sqrt{a}-1)^{2} / 4\right)\left(x_{1}^{2}-(\sqrt{a}+1)^{2} / 4\right)}\right\} \\
\quad+\frac{\sqrt{a}\left(4 x_{1}^{2}-a+1\right)\left(-6 x_{1}^{2}+a-1\right)}{16 x_{1} \sqrt{5 x_{1}^{2}+5-a}\left(x_{1}^{2}-(\sqrt{a}-1)^{2} / 4\right)\left(x_{1}^{2}-(\sqrt{a}+1)^{2} / 4\right)}
\end{gathered}
$$

and $x_{1}^{0}$ is a constant. 0 .

Notice that (148) decreases exponentially when approaching the "turning point" $j_{1}=$ 
Now we want to construct the asymptotics of the coefficients $\zeta_{j}$ near the "turning point" when $\left|j_{1}\right| \lesssim|m|^{3 / 5}$. For such $j_{1}$, the three-term recurrence relation (135) is approximately

$$
\begin{array}{r}
\left(1-\frac{4 \sqrt{a} j_{1}}{|m|(a-1)}\right) \zeta_{j-1}+\left(-2+\frac{4 \sqrt{a}}{|m|(a-1)}+\frac{4 \sqrt{5-a}}{|m|(a-1)}\left(k+\frac{1}{2}\right)+\frac{20 j_{1}^{2}}{|m|^{2}(a-1)}\right) \zeta_{j} \\
+\left(1+\frac{4 \sqrt{a} j_{1}}{|m|(a-1)}\right) \zeta_{j+1} \approx 0
\end{array}
$$

where the numbers $j$ and $j_{1}$ are related by (145). Let

$$
\zeta_{j}=\exp \left(-\frac{2 \sqrt{a} j_{1}^{2}}{(a-1)|m|}\right) \vartheta_{j}
$$

Then the coefficients $\vartheta_{j}$ approximately satisfy the three-term recurrence relation

$$
\vartheta_{j-1}+\left(-2+\frac{4 \sqrt{5-a}}{|m|(a-1)}\left(k+\frac{1}{2}\right)-\frac{4(5-a) j_{1}^{2}}{(a-1)^{2}|m|^{2}}\right) \vartheta_{j}+\vartheta_{j+1} \approx 0,
$$

which is a difference-equation analog of the Weber differential equation. Indeed, if $\vartheta(\tau) \in$ $C^{4}$, then, as $h \rightarrow 0$,

$$
\frac{\vartheta(\tau-h)-2 \vartheta(\tau)+\vartheta(\tau+h)}{h^{2}}=\vartheta^{\prime \prime}(\tau)+O\left(h^{2}\right) .
$$

Therefore, the principal term of the asymptotics $\vartheta_{j}^{(0)}$ can be represented in the form

$$
\vartheta_{j}^{(0)}=\vartheta\left(\frac{2 \sqrt[4]{5-a}}{\sqrt{a-1}} \frac{j_{1}}{\sqrt{|m|}}\right)
$$

where $\vartheta(\tau)$ is a solution of the Weber equation

$$
\frac{d^{2} \vartheta(\tau)}{d \tau^{2}}+\left(-\frac{\tau^{2}}{4}+k+\frac{1}{2}\right) \vartheta(\tau)=0
$$

The solutions of (151) can be expressed in terms of parabolic cylinder functions:

$$
\vartheta=c_{2} D_{k}(\tau)+c_{3} D_{-k-1}(i \tau),
$$

where $c_{2}$ and $c_{3}$ are constants. Using the formulas (100) and (101), we match the expansion at $j_{1}$ of order $-|m|^{3 / 5}$. Since $\zeta_{j,-}^{(0)}$ decreases exponentially, we have that $c_{3}=0$ in (152). We also have

\section{Lemma 15.}

$$
\zeta_{j}=c_{2} \exp \left(-\frac{2 \sqrt{a} j_{1}^{2}}{(a-1)|m|}\right) D_{k}\left(\frac{2 \sqrt[4]{5-a}}{\sqrt{a-1}} \frac{j_{1}}{\sqrt{|m|}}\right)\left(1+O\left(|m|^{-1 / 5}\right)\right) .
$$

Here $\left|j_{1}\right| \lesssim|m|^{3 / 5}$ as $|m| \rightarrow \infty$.

Furthermore, for $j_{1}$ of order $|m|^{3 / 5}$, the right-hand side of (153) matches $c_{4} \zeta_{j,-}^{(0)}$, where $c_{4}$ is a constant and $\zeta_{j,-}^{(0)}$ is determined by (148). This formula yields the asymptotics of $\zeta_{j}$ when $|m|^{3 / 5} \lesssim j_{1}$ and $|m|^{1 / 3} \lesssim j_{2}$, where

$$
j_{2}=n-|m|-j \text {. }
$$

Finally, similarly to (147), one can show that

$$
\zeta_{j}=\frac{|m|^{j_{2}-1}}{\left(j_{2}-1\right) !}\left(\frac{(\sqrt{a}-1)(5-\sqrt{a})}{4}\right)^{j_{2}-1} \zeta_{n-|m|-1}\left(1+O\left(\frac{j_{2}^{2}}{|m|}\right)\right)
$$

when $1 \leq j_{2} \lesssim|m|^{1 / 3}$. Here, the numbers $j_{2}$ and $j$ are related by (154). 
To find a relation between $\zeta_{0}$ and $\zeta_{n-|m|-1}$, it remains to express the constants in the expansions in terms of each other. This can be done in the process of matching the asymptotics. In the resulting formula, one then has an exponential multiplier whose exponent contains integrals of $f_{0}$ and $f_{1}$. But since the functions (149) and (150) are odd and the limits of integration are centrally symmetric with respect to zero, those integrals are zero. Since the principal terms of the asymptotics $D_{k}(\tau)$ when $\tau \rightarrow \pm \infty$ differ only by a factor $(-1)^{k}$, we have

\section{Theorem 3.}

$$
\zeta_{n-|m|-1}=(-1)^{k} \zeta_{0}\left(1+O\left(|m|^{-1 / 5}\right)\right), \quad|m| \rightarrow \infty .
$$

Since, by $(92), \Phi_{-}^{W K B}(\bar{z})=(-1)^{k} \zeta_{0} \bar{z}^{n-|m|-1}\left(1+O\left(\frac{1}{|m|}\right)+O\left(\frac{|m|}{\bar{z}}\right)\right)$, where $|\bar{z}| \gg|m|$, and the coefficient $\zeta_{0}$ is of the form (146), it follows from (131), (134), and (155) that $N(\bar{z})$ is small as compared to $\Phi_{-}^{W K B}(\bar{z})$ for $|\bar{z}| \gg|m|$ as well.

\section{The ASYMPtotics OF The NORM OF $\Phi(\bar{z})$}

Suppose the polynomial $\Phi(\bar{z})$ is given by $(57)$, where the function $p(\bar{u})$ is the asymptotic solution of the multipoint spectral problem. Let us compute the asymptotics of the norm of $\Phi(\bar{z})$ in $\mathcal{P}[m, n]$.

First, we determine the asymptotics of $\varrho(r)$ as $|m| \rightarrow \infty$. The function $\varrho(r)$ solves the problem (21), (22), and the desired asymptotics coincides with the WKB approximation $\varrho^{W K B}(r)$ of the solution of that problem. Notice that when $a>1$ and $r \geq 0$, the equation (21) has no turning points.

Lemma 16. Suppose the conditions (30) and (31) hold. If $r \ll|m|$, then

$$
\varrho(r)=\varrho^{W K B}(r)\left(1+O\left(\frac{1}{|m|}\right)+O\left(\frac{r}{|m|}\right)\right),
$$

where

$$
\begin{gathered}
\varrho^{W K B}(r)=\frac{c^{*}\left(\sqrt{\Lambda_{1}(r)}+r+1\right)^{|m|}}{\sqrt[4]{\Lambda_{1}(r)}\left(\sqrt{\Lambda_{1}(r)}+\sqrt{a}(r+1)\right)^{n+1 / 2}}, \\
\Lambda_{1}(r)=r^{2}+(4 a-2) r+1
\end{gathered}
$$

and

$$
c^{*}=\frac{|m| \sqrt{1+\sqrt{a}} \sqrt{a}(\sqrt{a}-1)}{2 \pi}\left(\frac{(1+\sqrt{a})^{\sqrt{a}}}{2}\right)^{|m|} .
$$

Moreover, if $r \gg|m|$, then

$$
\varrho(r)=\frac{n(n-|m|)}{2 \pi|m|} r^{-n+|m|-1}\left(1+O\left(\frac{|m|}{r}\right)\right) .
$$

Proof. Consider the function $\varrho(r)$ given by (20). Using the integral representation of the hypergeometric function 22, we have

$$
\varrho(r)=\frac{n(n-|m|)(|m|+1)_{n} \Gamma(2 n+2) r^{|m|}}{2 \pi(n+1)_{n+1} \Gamma(n+|m|+1) \Gamma(n-|m|+1)} E(r) .
$$

Here

$$
E(r)=\int_{0}^{1} \frac{t^{n+|m|}(1-t)^{n-|m|}}{(1-t(1-r))^{n+1}} d t=\int_{0}^{1} \frac{e^{|m| \Phi(t)}}{1-t(1-r)} d t
$$

and

$$
\Phi(t)=(\sqrt{a}+1) \ln t+(\sqrt{a}-1) \ln (1-t)-\sqrt{a} \ln (1-t(1-r)) .
$$


The asymptotics of $E(r)$ as $|m| \rightarrow \infty$ can be determined by the Laplace method [33]. The equation

$$
\Phi^{\prime}(t)=\frac{\sqrt{a}(1-r) t^{2}+(-2 \sqrt{a}-1+r) t+\sqrt{a}+1}{t(1-t)(1-t(1-r))}=0
$$

has a root

Since

$$
t_{*}=\frac{2 \sqrt{a}+1-r-\sqrt{\Lambda_{1}(r)}}{2 \sqrt{a}(1-r)} \in(0,1)
$$

(160)

$$
\Phi^{\prime \prime}\left(t_{*}\right)=\frac{2 t_{*} \sqrt{a}(1-r)-2 \sqrt{a}-1+r}{t_{*}\left(1-t_{*}\right)\left(1-t_{*}(1-r)\right)}=-\frac{\sqrt{\Lambda_{1}(r)}\left(\sqrt{\Lambda_{1}(r)}+\sqrt{a}(r+1)\right) 4 a^{3 / 2}}{(a-1)\left(\sqrt{\Lambda_{1}(r)}+r-1\right)^{2}}<0,
$$

we have that, when $t=t_{*}$, the function $\Phi(t)$ attains a maximum, and that maximum is the only one on $[0,1]$.

Now we use the relations

$$
\begin{gathered}
\frac{\left(2 \sqrt{a}+1-r-\sqrt{\Lambda_{1}(r)}\right)\left(-2 \sqrt{a} r+r-1-\sqrt{\Lambda_{1}(r)}\right)}{4 \sqrt{a}(\sqrt{a}-1)(r-1)}=\frac{\sqrt{\Lambda_{1}(r)}+r+1}{2(\sqrt{a}-1)}, \\
\frac{(1-r)^{2}\left(r-1+\sqrt{\Lambda_{1}(r)}\right)}{\left(2 \sqrt{a}+1-r-\sqrt{\Lambda_{1}(r)}\right)\left(-2 \sqrt{a} r+r-1+\sqrt{\Lambda_{1}(r)}\right)}=\frac{\sqrt{\Lambda_{1}(r)}+\sqrt{a}(r+1)}{2(a-1)},
\end{gathered}
$$

which show that

$$
r^{|m|} e^{|m| \Phi\left(t_{*}\right)}=\frac{1}{(2 \sqrt{a})^{n}}\left(\frac{\sqrt{\Lambda_{1}(r)}+r+1}{2(\sqrt{a}-1)}\right)^{|m|}\left(\frac{2(a-1)}{\sqrt{\Lambda_{1}(r)}+\sqrt{a}(r+1)}\right)^{n} .
$$

Finally, using the Laplace method with (160) and (161) and applying the Stirling formula, we have

$$
\begin{array}{r}
\varrho(r)=\frac{n(n-|m|) \Gamma(n+1) r^{|m|}}{2 \pi \Gamma(|m|+1) \Gamma(n-|m|+1)} \sqrt{-\frac{2 \pi}{|m| \Phi^{\prime \prime}\left(t_{*}\right)}} e^{|m| \Phi\left(t_{*}\right)}\left[\frac{2 \sqrt{a}}{\sqrt{\Lambda_{1}(r)}+r-1}\right. \\
\left.+O\left(\frac{1}{|m|}\right)\right]=\varrho^{W K B}(r)\left(1+O\left(\frac{1}{|m|}\right)+O\left(\frac{r}{|m|}\right)\right) .
\end{array}
$$

Here $r \ll|m|$.

The asymptotics (157) can be obtained from (159) if we expand, for $r \gg|m|$, the function

$$
\begin{aligned}
E(r)=\frac{1}{r^{n+1}} \int_{0}^{1} t^{|m|-1}(1-t)^{n-|m|} d t & \left(1+O\left(\frac{|m|}{r}\right)\right) \\
& =\frac{\Gamma(|m|) \Gamma(n-|m|+1)}{\Gamma(n+1) r^{n+1}}\left(1+O\left(\frac{|m|}{r}\right)\right)
\end{aligned}
$$

and then substitute (162) in (158).

We rewrite the principal terms of the WKB approximations (118) and (156) in the form

$$
\Phi_{-, 0}^{W K B}(\bar{z})=c_{-} t(\bar{z}) e^{|m| S(\bar{z})}, \quad \varrho^{W K B}\left(|z|^{2}\right)=c^{*} t_{1}\left(|z|^{2}\right) e^{|m| S_{1}\left(|z|^{2}\right)} .
$$

Here

$$
\begin{gathered}
S(\bar{z})=\sqrt{a} \ln (\sqrt{\Lambda(\bar{z})}+\sqrt{a}(\bar{z}+1))-\ln (\sqrt{\Lambda(\bar{z})}+\bar{z}+1), \\
S_{1}(r)=\ln \left(\sqrt{\Lambda_{1}(r)}+r+1\right)-\sqrt{a} \ln \left(\sqrt{\Lambda_{1}(r)}+\sqrt{a}(r+1)\right) .
\end{gathered}
$$


After substituting the functions (163) in the formula (19) for the scalar product, we have an integral

$$
\left\|\Phi_{-, 0}^{W K B}\right\|_{\mathcal{P}[m, n]}^{2}=\int_{\mathbb{C}}\left|c_{-}\right|^{2} c^{*}|t(\bar{z})|^{2} t_{1}\left(|z|^{2}\right) e^{|m| \Omega(\bar{z}, z)} d \bar{z} d z\left(1+O\left(|m|^{-1}\right)\right),
$$

where

$$
\Omega(\bar{z}, z)=S(\bar{z})+S(z)+S_{1}\left(|z|^{2}\right) .
$$

Notice that this function is continuous for all values of the arguments, but it is not differentiable on the arc $\smile \bar{z}_{-}, \bar{z}_{+}$.

Let us find the point where $\Omega(\bar{z}, z)$ attains a global maximum. Then the asymptotics of the integral (166) will be equal to the integral over a small neighborhood of that point.

First, we need

Lemma 17. The only critical point of $\Omega(\bar{z}, z)$ is $\bar{z}=z=1$.

Proof. Differentiating (164) and (165) we have that the critical points satisfy the system

$$
\begin{aligned}
& \frac{\partial \Omega}{\partial \bar{z}}=0 \\
& \frac{\partial \Omega}{\partial z}=0
\end{aligned}
$$

where

$$
\begin{aligned}
& \frac{\partial \Omega}{\partial \bar{z}}=\frac{(2 \sqrt{a}-1) \bar{z}^{2}+3(\sqrt{a}-1) \bar{z}-1-(\bar{z}-1) \sqrt{\Lambda(\bar{z})}}{2 \bar{z}\left(\bar{z}^{2}+3 \bar{z}+1\right)}-\frac{2 \sqrt{a}(\sqrt{a}-1) z}{\sqrt{\Lambda_{1}(z \bar{z})}+z \bar{z}(2 \sqrt{a}-1)+1}, \\
& (171) \\
& \frac{\partial \Omega}{\partial z}=\frac{(2 \sqrt{a}-1) z^{2}+3(\sqrt{a}-1) z-1-(z-1) \sqrt{\Lambda(z)}}{2 z\left(z^{2}+3 z+1\right)}-\frac{2 \sqrt{a}(\sqrt{a}-1) \bar{z}}{\sqrt{\Lambda_{1}(z \bar{z})}+z \bar{z}(2 \sqrt{a}-1)+1} .
\end{aligned}
$$

The point $\bar{z}=z=1$ satisfies both (168) and (169). Now we show that the system (168), (169) has no other solutions.

First, notice that for $\bar{z}=z=\bar{z}_{j}$, where $\bar{z}_{j}, j=1,2,3$, are the singular points (51), the values of the derivatives $\partial \Omega / \partial \bar{z}$ and $\partial \Omega / \partial z$ computed by passing to the limit in (170) and (171) are nonzero. Moreover, the point $\bar{z}=z=-1$ is not critical because $\bar{z}=-1$ lies on the arc $\smile \bar{z}_{-}, \bar{z}_{+}$, where $\Omega(\bar{z}, z)$ is not differentiable.

Rewriting (168) in the form

$$
\frac{2 \sqrt{a} \bar{z}^{2}+3 \sqrt{a} \bar{z}-(\bar{z}-1) \sqrt{\Lambda(\bar{z})}}{\bar{z}^{2}+3 \bar{z}+1}=\frac{2 \sqrt{a} z \bar{z}-\sqrt{\Lambda_{1}(z \bar{z})}}{z \bar{z}-1}
$$

and using the substitution $\bar{u}=\bar{z}-1, u=z-1$, we have

$$
\begin{aligned}
\sqrt{a}(\bar{u}+1)(3 u \bar{u}+5 u+ & 5 \bar{u}+10)+(u \bar{u}+u+\bar{u}) \bar{u} \sqrt{\bar{u}^{2}+(5-a) \bar{u}+5-a} \\
& =\left(\bar{u}^{2}+5 \bar{u}+5\right) \sqrt{(u \bar{u}+u+\bar{u})^{2}+4 a(u \bar{u}+u+\bar{u}+1)} .
\end{aligned}
$$

Squaring both sides of (172), we have

$$
\begin{aligned}
(\bar{u}+1)\{ & \left\{a \left[u^{2}(\bar{u}+1)\left(-\bar{u}^{3}+8 \bar{u}^{2}+30 \bar{u}+25\right)-2 u \bar{u}\left(3 \bar{u}^{3}+6 \bar{u}^{2}-5\right)\right.\right. \\
& \left.\left.-\quad 5 \bar{u}^{2}\left(\bar{u}^{2}+3 \bar{u}+3\right)\right]-5\left(\bar{u}^{2}+5 \bar{u}+5\right)(u \bar{u}+u+\bar{u})^{2}\right\} \\
= & -(\bar{u}+1) 2 \sqrt{a}(3 u \bar{u}+5 u+5 \bar{u}+10)(u \bar{u}+u+\bar{u}) \bar{u} \sqrt{\bar{u}^{2}+(5-a)(\bar{u}+1)} .
\end{aligned}
$$


Now divide (173) by $\bar{u}+1$ and square both sides. After factoring the result, we have an equation

$$
\begin{aligned}
(a-1)(u \bar{u}+u+\bar{u})^{2}\left(\bar{u}^{2}+5 \bar{u}+5\right)^{2}\left\{a\left[u^{2}(\bar{u}+5)^{2}+10 u \bar{u}(\bar{u}-3)+25 \bar{u}^{2}\right]\right. & \\
- & \left.25(u \bar{u}+u+\bar{u})^{2}\right\}=0 .
\end{aligned}
$$

Since the system (168), (169), when $\bar{z}=z= \pm 1$ and $\bar{z}=z=\bar{z}_{j}, j=2,3$, was already examined and $a \neq 1$, we divide (174) by

$$
(a-1)(u \bar{u}+u+\bar{u})^{2}\left(\bar{u}^{2}+5 \bar{u}+5\right)^{2} .
$$

Passing to the polar coordinates $\bar{u}=\rho e^{i \varphi}, u=\rho e^{-i \varphi}$ and canceling out $\rho^{2}$, we have a quadratic, with respect to $\rho$, equation

$$
(a-25) \rho^{2}+(20 a-100) \rho \cos \varphi+100(a-1) \cos ^{2} \varphi-80 a=0 .
$$

Since its discriminant $d$ satisfies the inequality

$$
d=320 a\left(20 \cos ^{2} \varphi+a-25\right) \leq 320 a(a-5),
$$

we have that $d<0$ for $1<a<5$, and therefore the function $\Omega(\bar{z}, z)$ has no critical points other than $\bar{z}=z=1$.

Theorem 4. The function $\Omega(\bar{z}, z)$ attains its maximum value at $\bar{z}=z=1$.

Proof. Since

$$
\Omega(x, x)=\sqrt{a} \ln (\sqrt{a}+1)-\ln 2+\frac{(\sqrt{a}-1)(5-\sqrt{a})}{2 x}+O\left(\frac{1}{x^{2}}\right), \quad x \rightarrow \infty,
$$

the function $\Omega(x, x)$ decreases for sufficiently large positive $x$ and, therefore, does not have a maximum at infinity. Moreover, if $\bar{z}=e^{i \varphi} \in \smile \bar{z}_{-}, \bar{z}_{+}$, then

$$
\Omega(\bar{z}, z)=\sqrt{a} \ln (3+2 \cos \varphi)+\sqrt{a} \ln \left(\frac{a-1}{4 \sqrt{a}}\right)-\ln \left(\frac{\sqrt{a}-1}{2}\right)
$$

is less than the value of $\Omega$ at $\bar{z}=z=1$, which equals

$$
\Omega(1,1)=\sqrt{a} \ln \frac{(\sqrt{5-a}+2 \sqrt{a})^{2}}{4 \sqrt{a}}+\ln \frac{2(\sqrt{a}+1)}{(\sqrt{5-a}+2)^{2}} .
$$

Indeed, it follows from (175) that, for $\bar{z} \in \smile \bar{z}_{-}, \bar{z}_{+}$, the function $\Omega(\bar{z}, z)$ has a maximum at the points $\bar{z}_{ \pm}$. On the other hand, $S_{1}\left(|z|^{2}\right)$ is constant on the circle $|\bar{z}|=1$ and, by (130),

$$
\Omega\left(\bar{z}_{ \pm}, z_{ \pm}\right)<\Omega(1,1) .
$$

Thus, it remains to check that $\Omega(\bar{z}, z)$ has a local maximum at its only critical point $\bar{z}=z=1$. Thus we need to check that

$$
\frac{\partial^{2} \Omega}{\partial x^{2}}=\frac{\partial^{2} \Omega}{\partial \bar{z}^{2}}+2 \frac{\partial^{2} \Omega}{\partial \bar{z} \partial z}+\frac{\partial^{2} \Omega}{\partial z^{2}}<0, \quad D=\left(\frac{\partial^{2} \Omega}{\partial \bar{z} \partial z}\right)^{2}-\frac{\partial^{2} \Omega}{\partial \bar{z}^{2}} \frac{\partial^{2} \Omega}{\partial z^{2}}>0 .
$$

Differentiating the functions (170) and (171), we have

$$
\frac{\partial^{2} \Omega}{\partial \bar{z}^{2}}(1,1)=\frac{\partial^{2} \Omega}{\partial z^{2}}(1,1)=\frac{(\sqrt{5-a}-2 \sqrt{a})^{2}}{40 \sqrt{a}}, \quad \frac{\partial^{2} \Omega}{\partial \bar{z} \partial z}(1,1)=\frac{1-a}{8 \sqrt{a}} .
$$

Therefore, for $1<a<5$,

$$
\frac{\partial^{2} \Omega}{\partial x^{2}}=\frac{\sqrt{5-a}(\sqrt{5-a}-2 \sqrt{a})}{10 \sqrt{a}}<0, \quad D=\frac{\sqrt{5-a}(\sqrt{5-a}-2 \sqrt{a})^{2}}{200 \sqrt{a}}>0 .
$$


By Theorem 4, the main contribution to the norm of the asymptotic solution of the multipoint spectral problem $p(\bar{z})$ comes from a small neighborhood of $\bar{z}=z=1$. Since $p(\bar{z})$ is given by (112) near $\bar{z}=1$, let us substitute it in the formula (19) for the scalar product and compute the asymptotics of the resulting integral.

Let

$$
\theta(t, r, a)=\exp \left(-\left(1-\sqrt{\frac{5-a}{4 a}}\right) t^{2}-\left(\sqrt{\frac{4 a}{5-a}}-1\right) r^{2}\right)
$$

\section{Lemma 18.}

$$
\begin{gathered}
\|p(\bar{z})\|_{\mathcal{P}[m, n]}^{2}=\frac{\alpha_{1}^{2} \sqrt{\sqrt{a}+1}(\sqrt{a}-1)}{2^{k+1 / 2} \pi \sqrt{5-a}}\left(\frac{5^{\sqrt{a}}(\sqrt{a}+1)^{\sqrt{a}+1}}{(4 \sqrt{a})^{\sqrt{a}}}\right)^{|m|} \Sigma_{0}(a)\left(1+O\left(\frac{1}{|m|}\right)\right), \\
|m| \rightarrow \infty
\end{gathered}
$$

where

$$
\Sigma_{0}(a)=\int_{\mathbb{R}^{2}} \theta(t, r, a)\left|H_{k}(t+i r)\right|^{2} d t d r .
$$

Proof. Expanding $\varrho^{W K B}\left(|z|^{2}\right)$ by the powers of $\bar{u}$ and $u$, where $\bar{u}$ and $\bar{z}$ are related by (94) and (95), we have

$$
\begin{aligned}
& \varrho^{W K B}\left(|z|^{2}\right)=\frac{c^{*}}{2^{3 / 2} \sqrt{a}}\left(\frac{2(\sqrt{a}+1)}{(4 \sqrt{a})^{\sqrt{a}}}\right)^{|m|} \exp \left(-\frac{\sqrt{|m|}(\sqrt{a}-1) \beta(\bar{u}+u)}{2}\right. \\
& \left.+\frac{(\sqrt{a}-1) \beta^{2}}{8 \sqrt{a}}\left\{-(\sqrt{a}+1)|\bar{u}|^{2}+(3 \sqrt{a}-1) \frac{\left(\bar{u}^{2}+u^{2}\right)}{2}\right\}\right)\left\{1-\frac{\beta(\bar{u}+u)}{2 \sqrt{|m|}}\right. \\
& \left.+\frac{(\sqrt{a}-1) \beta^{3}}{\sqrt{|m|} 16 \sqrt{a}}\left[(3-5 \sqrt{a}) \frac{\bar{u}^{3}+u^{3}}{3}+(\sqrt{a}+1)|\bar{u}|^{2}(\bar{u}+u)\right]+O\left(\frac{|u|^{4}+1}{|m|}\right)\right\} .
\end{aligned}
$$

Substituting (112) and (179) in (19), we then have

$$
\begin{aligned}
& \|p(\bar{z})\|_{\mathcal{P}[m, n]}^{2}=\frac{\alpha_{1}^{2} \mu^{2} c^{*}}{2^{3 / 2} \sqrt{a}}\left(\frac{2(\sqrt{a}+1)}{(4 \sqrt{a}) \sqrt{a}}\right)^{|m|} \int_{\mathbb{C}} \exp \left(-\frac{5(a-1)|\bar{u}|^{2}}{8 \sqrt{a} \sqrt{5-a}}\right. \\
& \left.+\left(-\frac{1}{4}+\frac{3 a+5}{16 \sqrt{a} \sqrt{5-a}}\right)\left(\bar{u}^{2}+u^{2}\right)\right)\left\{\left|H_{k}\left(\frac{\bar{u}}{\sqrt{2}}\right)\right|^{2}+\frac{\sqrt{5}}{\sqrt{|m|} \sqrt[4]{5-a}}\{[-\bar{u}-u\right. \\
& \left.+\frac{(-9 a+12 \sqrt{a} \sqrt{5-a}-15)\left(\bar{u}^{3}+u^{3}\right)}{48 \sqrt{a} \sqrt{5-a}}+\frac{5(a-1)|\bar{u}|^{2}(\bar{u}+u)}{16 \sqrt{a} \sqrt{5-a}}\right]\left|H_{k}\left(\frac{\bar{u}}{\sqrt{2}}\right)\right|^{2} \\
& \left.\left.-\frac{1}{2 \sqrt{2}}\left[\bar{u}^{2} H_{k}^{\prime}\left(\frac{\bar{u}}{\sqrt{2}}\right) H_{k}\left(\frac{u}{\sqrt{2}}\right)+u^{2} H_{k}^{\prime}\left(\frac{u}{\sqrt{2}}\right) H_{k}\left(\frac{\bar{u}}{\sqrt{2}}\right)\right]\right\}\right\} d \bar{z} d z\left(1+O\left(\frac{1}{|m|}\right)\right) .
\end{aligned}
$$

Define real variables $t$ and $r$ by the formula

$$
\frac{\bar{u}}{\sqrt{2}}=t+i r
$$

Then

$$
\begin{aligned}
d \bar{z} d z=\frac{20 d t d r}{|m| \sqrt{5-a}}, \quad \bar{u} & +u=2 \sqrt{2} t, \quad \bar{u}^{3}+u^{3}=4 \sqrt{2}\left(t^{3}-3 t r^{2}\right) \\
|\bar{u}|^{2}=2\left(t^{2}+r^{2}\right), \quad \bar{u}^{2} H_{k}^{\prime}\left(\frac{\bar{u}}{\sqrt{2}}\right) H_{k} & \left(\frac{u}{\sqrt{2}}\right)+u^{2} H_{k}^{\prime}\left(\frac{u}{\sqrt{2}}\right) H_{k}\left(\frac{\bar{u}}{\sqrt{2}}\right) \\
= & 2\left(t^{2}-r^{2}\right) \frac{\partial}{\partial t}\left|H_{k}(t+i r)\right|^{2}+4 t r \frac{\partial}{\partial r}\left|H_{k}(t+i r)\right|^{2}
\end{aligned}
$$


and (180) rewrites as

$$
\begin{gathered}
\|p(\bar{z})\|_{\mathcal{P}[m, n]}^{2}=\frac{\alpha_{1}^{2} \mu^{2} 5 \sqrt{2} c^{*}}{|m| \sqrt{a} \sqrt{5-a}}\left(\frac{2(\sqrt{a}+1)}{(4 \sqrt{a}) \sqrt{a}}\right)^{|m|} \int_{\mathbb{R}^{2}} \theta(t, r, a)\left\{\left|H_{k}(t+i r)\right|^{2}\right. \\
+\frac{\sqrt{5}}{\sqrt{|m|} \sqrt[4]{5-a}}\left\{\left[-2 \sqrt{2} t+\frac{(-9 a+12 \sqrt{a} \sqrt{5-a}-15)\left(t^{3}-3 t r^{2}\right)}{6 \sqrt{2} \sqrt{a} \sqrt{5-a}}\right.\right. \\
\left.+\frac{5(a-1)\left(t^{2}+r^{2}\right) t}{2 \sqrt{2} \sqrt{a} \sqrt{5-a}}\right]\left|H_{k}(t+i r)\right|^{2} \\
\left.\left.-\frac{1}{\sqrt{2}}\left[\left(t^{2}-r^{2}\right) \frac{\partial}{\partial t}\left|H_{k}(t+i r)\right|^{2}+2 t r \frac{\partial}{\partial r}\left|H_{k}(t+i r)\right|^{2}\right]\right\}\right\} d t d r\left(1+O\left(\frac{1}{|m|}\right)\right) .
\end{gathered}
$$

In (182), the summands of order $|m|^{-1 / 2}$ are integrals of odd functions with symmetric limits. Therefore, they are equal to zero.

Now consider the norm of $N(\bar{z})$.

Lemma 19. As $|m| \rightarrow \infty$,

$$
\|N(\bar{z})\|_{\mathcal{P}[m, n]}=O\left(|m|^{-1 / 4}\left|c_{-}\right|\left(a^{\sqrt{a}}(a-1)^{\sqrt{a}-1}\right)^{|m| / 2}\right) .
$$

Proof. It follows from the formulas (19) and (121) and from the inequality (129) that

(184) $\quad\|N(\bar{z})\|_{\mathcal{P}[m, n]}^{2}$

$$
\begin{aligned}
=\sum_{ \pm} O & \left(\frac{\left|c_{-}\right|^{2}\left(a^{\sqrt{a}}(a-1)^{\sqrt{a}-1}\right)^{|m|}}{|m|^{3 / 2}} \int_{\left|\bar{z}-\bar{z}_{ \pm}\right|>|m|^{-1}} \frac{|\bar{z}|^{2(\sqrt{a}-1)|m|} \varrho^{W K B}\left(|z|^{2}\right) d \bar{z} d z}{\left|\bar{z}-\bar{z}_{ \pm}\right|^{2}}\right) \\
& +\sum_{ \pm} O\left(|m|^{1 / 2}\left|c_{-}\right|^{2}\left(a^{\sqrt{a}}(a-1)^{\sqrt{a}-1}\right)^{|m|} \int_{\left|\bar{z}-\bar{z}_{ \pm}\right|<|m|^{-1}} \varrho^{W K B}\left(|z|^{2}\right) d \bar{z} d z\right) .
\end{aligned}
$$

Let us use the substitution $\bar{z}=\sqrt{r} e^{i \varphi}, \bar{z}_{ \pm}=e^{i \varphi_{ \pm}}$. Since

$$
\int_{0}^{2 \pi} \frac{d \varphi}{\left|\bar{z}-\bar{z}_{ \pm}\right|^{2}}=\int_{0}^{2 \pi} \frac{d \varphi}{r-2 \sqrt{r} \cos \left(\varphi-\varphi_{ \pm}\right)+1}=\frac{2 \pi}{|r-1|}
$$

(184) rewrites as

$$
\begin{array}{r}
\|N(\bar{z})\|_{\mathcal{P}[m, n]}^{2}=O\left(\frac{\left|c_{-}\right|^{2}\left(a^{\sqrt{a}}(a-1)^{\sqrt{a}-1}\right)^{|m|}}{|m|^{3 / 2}} \int_{|\sqrt{r}-1|>|m|^{-1}} \frac{r^{(\sqrt{a}-1)|m|} \varrho^{W K B}(r) d r}{|r-1|}\right) \\
+O\left(\frac{\left|c_{-}\right|^{2}\left(a^{\sqrt{a}}(a-1)^{\sqrt{a}-1}\right)^{|m|} \varrho^{W K B}(1)}{|m|^{3 / 2}}\right) .
\end{array}
$$

Now use the fact that the function

$$
\Pi(r)=S_{1}(r)+(\sqrt{a}-1) \ln r,
$$

where $S_{1}(r)$ is of the form (165), increases when $r>0$. Indeed,

$$
\frac{d \Pi(r)}{d r}=-\frac{2 \sqrt{a}(\sqrt{a}-1)}{\sqrt{\Lambda_{1}(r)}+(2 \sqrt{a}-1) r+1}+\frac{\sqrt{a}-1}{r}=\frac{(\sqrt{a}-1)\left(\sqrt{\Lambda_{1}(r)}-r+1\right)}{r\left(\sqrt{\Lambda_{1}(r)}+(2 \sqrt{a}-1) r+1\right)}>0 .
$$

Therefore the largest contribution to the integral in (185) comes from a neighborhood of $\infty$. As a result,

$$
\int_{|\sqrt{r}-1|>|m|^{-1}} \frac{r^{(\sqrt{a}-1)|m|} \varrho^{W K B}(r) d r}{|r-1|}=O\left(\int_{1}^{\infty} \frac{r^{(\sqrt{a}-1)|m|}|m| r^{-(\sqrt{a}-1)|m|} d r}{r^{2}}\right)=O(|m|) .
$$


Finally, since for $r>0$,

$$
\frac{d S_{1}(r)}{d r}=-\frac{2 \sqrt{a}(\sqrt{a}-1)}{\sqrt{\Lambda_{1}(r)}+(2 \sqrt{a}-1) r+1}<0,
$$

the function $S_{1}(r)$ decreases and, therefore,

$$
\varrho^{W K B}(1) \ll \varrho^{W K B}(0)=\frac{|m| \sqrt{a}(\sqrt{a}-1)}{2 \pi} .
$$

Substituting the estimates (186) and (187) in (185), we have (183). The lemma is proved.

It follows from (107) and (177) that

(188) $\|p(\bar{z})\|_{\mathcal{P}[m, n]}=$

$$
\begin{array}{r}
\frac{\left|c_{-}\right| 5^{(k+1) / 2} \sqrt[4]{\sqrt{a}+1} \sqrt{\sqrt{a}-1}}{|m|^{k / 2}(5-a)^{3(k+1) / 4} 2^{5(2 k+1) / 4} \sqrt{\pi}}\left(\frac{(\sqrt{5-a}+2 \sqrt{a})^{2 \sqrt{a}}(\sqrt{a}+1)^{\sqrt{a}+1}}{(\sqrt{5-a}+2)^{2}(4 \sqrt{a})^{\sqrt{a}}}\right)^{|m| / 2} \times \\
\left(\Sigma_{0}(a)\right)^{1 / 2}\left(1+O\left(\frac{1}{|m|}\right)\right), \quad|m| \rightarrow \infty .
\end{array}
$$

Therefore, by (188) and (183), $\|N(\bar{z})\|_{\mathcal{P}[m, n]}$ is exponentially small as compared to $\|p(\bar{z})\|_{\mathcal{P}[m, n]}$ on the interval $a \in\left(1, a_{0}\right)$, where $a_{0} \approx 3,03$, since for such $a$ we have

$$
a^{\sqrt{a}}(a-1)^{\sqrt{a}-1}<\frac{(\sqrt{5-a}+2 \sqrt{a})^{2 \sqrt{a}}(\sqrt{a}+1)^{\sqrt{a}+1}}{(\sqrt{5-a}+2)^{2}(4 \sqrt{a})^{\sqrt{a}}} .
$$

The fact that $\|N(\bar{z})\|_{\mathcal{P}[m, n]}$ is exponentially small on the entire interval $a \in(1,5)$ follows from (188) and the estimate

$$
\|N(\bar{z})\|_{\mathcal{P}[m, n]}=O\left(\left|c_{-}\right|\left(\frac{(\sqrt{a}+1)^{\sqrt{a}}}{2}\right)^{|m|}\right), \quad|m| \rightarrow \infty .
$$

Indeed, by Theorem 4,

$$
\Omega(\infty, \infty)<\Omega(1,1),
$$

and (167) allows us to rewrite (190) in the form

$$
\frac{(\sqrt{a}+1)^{2 \sqrt{a}}}{4}<\frac{(\sqrt{5-a}+2 \sqrt{a})^{2 \sqrt{a}}(\sqrt{a}+1)^{\sqrt{a}+1}}{(\sqrt{5-a}+2)^{2}(4 \sqrt{a})^{\sqrt{a}}} .
$$

We remark that the estimate (189) is obtained similarly to (183). One needs to use the asymptotics of the coefficients $\zeta_{j}$ of the power series (132), which can be found using the discrete WKB method (see $\S 9$ ).

Finally, using (120), (125), (177), and the Cauchy-Bunyakovsky inequality, we have that the asymptotics of the norm of $\Phi(\bar{z})$ as $|m| \rightarrow \infty$ is of the form

$$
\begin{aligned}
& \|\Phi(\bar{z})\|_{\mathcal{P}[m, n]} \\
& =\left(\|p(\bar{z})\|_{\mathcal{P}[m, n]}^{2}+O\left(\|p(\bar{z})\|_{\mathcal{P}[m, n]}\|N(\bar{z})\|_{\mathcal{P}[m, n]}\right)+O\left(\|N(\bar{z})\|_{\mathcal{P}[m, n]}^{2}\right)\right)^{1 / 2} \\
& \quad=\frac{\alpha_{1} \sqrt[4]{\sqrt{a}+1} \sqrt{\sqrt{a}-1}}{2^{k / 2+1 / 4} \sqrt{\pi} \sqrt[4]{5-a}}\left(\frac{5^{\sqrt{a}}(\sqrt{a}+1)^{\sqrt{a}+1}}{(4 \sqrt{a})^{\sqrt{a}}}\right)^{|m| / 2}\left(\Sigma_{0}(a)\right)^{1 / 2}\left(1+O\left(\frac{1}{|m|}\right)\right) .
\end{aligned}
$$

Here $\Sigma_{0}(a)$ is given by (178). 
Thus, if the constant $\alpha_{1}$ in (112) is

$$
\alpha_{1}=\frac{2^{k / 2+1 / 4} \sqrt{\pi} \sqrt[4]{5-a}}{\sqrt[4]{\sqrt{a}+1} \sqrt{\sqrt{a}-1}}\left(\frac{(4 \sqrt{a})^{\sqrt{a}}}{5^{\sqrt{a}}(\sqrt{a}+1)^{\sqrt{a}+1}}\right)^{|m| / 2}\left(\Sigma_{0}(a)\right)^{-1 / 2}\left(1+O\left(\frac{1}{|m|}\right)\right),
$$

then the condition $\|\Phi(\bar{z})\|_{\mathcal{P}[m, n]}=1+O\left(|m|^{-1}\right),|m| \rightarrow \infty$, is satisfied. The determination of the multiplier $\alpha_{1}$ in $p(\bar{z})$ finishes the construction of $\Phi(\bar{z})$.

\section{Summarizing theorems. Formulas For QUANTUm AVERAGeS}

Theorem 5. Let

$$
\tilde{\xi}_{k}=a+1+\frac{4}{|m|} \sqrt{5-a}\left(k+\frac{1}{2}\right)+O\left(\frac{1}{|m|^{2}}\right), \quad k=0,1,2, \ldots,
$$

and the polynomial $\Phi_{k}(\bar{z})$ be given by (57), where $p(\bar{u})$ is a solution of the multipoint spectral problem such that $\alpha_{1}$ is given by (192). Then, for $n=\sqrt{a}|m|$, where $1<$ $a<5$ and $|m| \rightarrow \infty, \tilde{\xi}_{k}$ and $\Phi_{k}(\bar{z})$ are an asymptotic eigenvalue and an asymptotic eigenfunction of the problem (48), (29) in the space $\mathcal{P}[m, n]$. More precisely, if $\tilde{\xi}_{k}$ is of the form (193), then the polynomial $\Phi_{k}(\bar{z})$ satisfies equation (48) with accuracy $O\left(|m|^{-2}\right)$ with an estimate of the residual in the norm of $\mathcal{P}[m, n]$, as well as the normalization condition (29) with accuracy $O\left(|m|^{-1}\right)$.

Proof. An estimate of the residual is obtained similarly to the computation of the asymptotics of the norm. The main contribution to the asymptotics of the integral comes from a small neighborhood of $\bar{z}=z=1$. Therefore, it suffices to estimate the residual near $\bar{z}=1$, where it has the form

$$
R=O\left((\bar{z}-1)^{4} p_{0}\right)+O\left(|m|^{-2} p_{0}\right)=O\left(|m|^{-2}\left(1+\left(t^{2}+r^{2}\right)^{2}\right) p_{0}\right) .
$$

Here $p_{0}$ is given by (113), and $t$ and $r$ by (181).

The asymptotics of $\|R\|_{\mathcal{P}[m, n]}$ contains the integral

$$
\int_{\mathbb{R}^{2}} \theta(t, r, a)\left(1+\left(t^{2}+r^{2}\right)^{2}\right)^{2}\left|H_{k}(t+i r)\right|^{2} d t d r
$$

instead of the function $\Sigma_{0}(a)$, as was the case in (191). Thus, $\|R\|_{\mathcal{P}[m, n]}=O\left(|m|^{-2}\right)$, $|m| \rightarrow \infty$.

The normalizing condition (29) holds by virtue of (191) and (192).

Notice that the operator $g_{0}(\stackrel{\circ}{B}): \mathcal{P}[m, n] \rightarrow \mathcal{P}[m, n]$, where $\stackrel{\circ}{B}=\left(\stackrel{\circ}{B}_{0}, \stackrel{\circ}{B}_{1}, \stackrel{\circ}{B}_{2}, \stackrel{\circ}{B}_{3}\right)$ and $g_{0}(b)$ is of the form (17), is Hermitian. Therefore, as is known [36], near the asymptotic eigenvalues $\tilde{\xi}_{k}$ there are points of the spectrum of the operator $g_{0}(\stackrel{B}{B})$.

Now we want to determine the asymptotic average values of differential operators. This problem was considered, for example, in [37 and [16]. The computation of the quantum averages is similar to that of the norm.

We begin by establishing the formula (47). Suppose $\Phi_{k}(\bar{z})$ is an asymptotic eigenfunction of the problem (48), (29), and the operators $\stackrel{\circ}{B}_{0}, \stackrel{\circ}{B}_{1}, \stackrel{\circ}{B}_{2}, \stackrel{\circ}{B}_{3}$ are given by $(27)$, where $\hbar=1 /|m|$ is a small parameter.

Lemma 20. As $|m| \rightarrow \infty$,

$$
\begin{aligned}
& \left(\stackrel{\circ}{B}_{0} \Phi_{k}, \Phi_{k}\right)_{\mathcal{P}[m, n]}=O\left(|m|^{-1}\right), \quad\left(\stackrel{\circ}{B}_{2} \Phi_{k}, \Phi_{k}\right)_{\mathcal{P}[m, n]}=\frac{a-1}{4}+O\left(|m|^{-1}\right), \\
& \left(\stackrel{\circ}{B}_{1} \Phi_{k}, \Phi_{k}\right)_{\mathcal{P}[m, n]}=O\left(|m|^{-1}\right), \quad\left(\stackrel{\circ}{B}_{3} \Phi_{k}, \Phi_{k}\right)_{\mathcal{P}[m, n]}=\frac{a+1}{4}+O\left(|m|^{-1}\right) .
\end{aligned}
$$


Proof. The main contribution to the asymptotics of the integrals for the averages also comes from a small neighborhood of $\bar{z}=z=1$, where for $\Phi_{k}(\bar{z})$ one has the expansion (112). Differentiating $p(\bar{z})$, we have

$$
\begin{aligned}
& \frac{1}{|m|} \frac{d p}{d \bar{z}}=\frac{(\sqrt{a}-1)}{2}\left(p_{0}+\frac{p_{1}}{\sqrt{|m|}}\right)+\frac{(\bar{z}-1)}{10}(5-3 \sqrt{a}-\sqrt{5-a}) p_{0} \\
& +\frac{\alpha_{1} \mu \sqrt[4]{5-a}}{\sqrt{|m|} \sqrt{10}} \exp \left(\frac{\sqrt{|m|}(\sqrt{a}-1) \sqrt{5} \bar{u}}{2 \sqrt[4]{5-a}}+\left(-1+\frac{5-3 \sqrt{a}}{\sqrt{5-a}}\right) \frac{\bar{u}^{2}}{4}\right) H_{k}^{\prime}\left(\frac{\bar{u}}{\sqrt{2}}\right) \\
& \quad+O\left(\frac{p_{0}}{|m|}\right)+O\left(\frac{\bar{u}^{6}}{|m|} p_{0}\right) \\
& \quad+\exp \left(\frac{\sqrt{|m|}(\sqrt{a}-1) \beta \bar{u}}{2}+\frac{(5-3 \sqrt{a}) \beta^{2} \bar{u}^{2}}{20}\right)\left(O\left(\frac{\bar{u}}{|m|} \frac{d y_{0}}{d \bar{u}}\right)+O\left(\frac{\bar{u}^{5}}{|m|} \frac{d y_{0}}{d \bar{u}}\right)\right) .
\end{aligned}
$$

Therefore,

$$
\begin{aligned}
& \left(\stackrel{\circ}{B}_{0} \Phi_{k}, \Phi_{k}\right)_{\mathcal{P}[m, n]}=\left(\stackrel{\circ}{B}_{0}\left(p_{0}+p_{1} / \sqrt{|m|}\right), p_{0}+p_{1} / \sqrt{|m|}\right)_{\mathcal{P}[m, n]}+O\left(|m|^{-1}\right) \\
& =\left((\sqrt{a}-1)\left(p_{0}+\frac{p_{1}}{\sqrt{|m|}}\right)+\frac{(\bar{z}-1)}{5}(5-3 \sqrt{a}-\sqrt{5-a}) p_{0}\right. \\
& +\frac{\alpha_{1} \mu \sqrt{2} \sqrt[4]{5-a}}{\sqrt{|m|} \sqrt{5}} \exp \left(\frac{\sqrt{|m|}(\sqrt{a}-1) \sqrt{5} \bar{u}}{2 \sqrt[4]{5-a}}+\left(-1+\frac{5-3 \sqrt{a}}{\sqrt{5-a}}\right) \frac{\bar{u}^{2}}{4}\right) H_{k}^{\prime}\left(\frac{\bar{u}}{\sqrt{2}}\right) \\
& \left.+(\bar{z}-1)(\sqrt{a}-1) p_{0}-(\sqrt{a}-1)\left(p_{0}+\frac{p_{1}}{\sqrt{|m|}}\right), p_{0}+\frac{p_{1}}{\sqrt{|m|}}\right)_{\mathcal{P}[m, n]}+O\left(\frac{1}{|m|}\right) \\
& =O\left(\frac{1}{|m|}\right) \text {. }
\end{aligned}
$$

In (196),

$$
\begin{gathered}
\int_{\mathbb{R}^{2}} \theta(t, r, a)(t+i r)\left|H_{k}(t+i r)\right|^{2} d t d r=0 \\
\int_{\mathbb{R}^{2}} \theta(t, r, a) H_{k}^{\prime}(t+i r) H_{k}(t-i r) d t d r \\
=\int_{\mathbb{R}^{2}} \theta(t, r, a) \frac{1}{2}\left(\frac{\partial}{\partial t}\left|H_{k}(t+i r)\right|^{2}-i \frac{\partial}{\partial r}\left|H_{k}(t+i r)\right|^{2}\right) d t d r=0,
\end{gathered}
$$

because in (197) and (198) we integrate odd functions with symmetric limits.

Likewise, since

$$
\begin{array}{r}
\frac{1}{|m|^{2}} \frac{d^{2} p}{d \bar{z}^{2}}=\frac{(\sqrt{a}-1)^{2}}{4}\left(p_{0}+\frac{p_{1}}{\sqrt{|m|}}\right)+\frac{(\bar{z}-1)(\sqrt{a}-1)}{10}(5-3 \sqrt{a}-\sqrt{5-a}) p_{0} \\
+\frac{\alpha_{1} \mu \sqrt[4]{5-a}(\sqrt{a}-1)}{\sqrt{|m|} \sqrt{10}} \exp \left(\frac{\sqrt{|m|}(\sqrt{a}-1) \sqrt{5} \bar{u}}{2 \sqrt[4]{5-a}}+\left(-1+\frac{5-3 \sqrt{a}}{\sqrt{5-a}}\right) \frac{\bar{u}^{2}}{4}\right) H_{k}^{\prime}\left(\frac{\bar{u}}{\sqrt{2}}\right) \\
+O\left(\frac{p_{0}}{|m|}\right)+O\left(\frac{\bar{u}^{6}}{|m|} p_{0}\right)+\exp \left(\frac{\sqrt{|m|}(\sqrt{a}-1) \beta \bar{u}}{2}+\frac{(5-3 \sqrt{a}) \beta^{2} \bar{u}^{2}}{20}\right)\left(O\left(\frac{\bar{u}}{|m|} \frac{d y_{0}}{d \bar{u}}\right)\right. \\
\left.+O\left(\frac{\bar{u}^{5}}{|m|} \frac{d y_{0}}{d \bar{u}}\right)\right),
\end{array}
$$

we have, by (197) and (198), 


$$
\begin{array}{r}
\left(\stackrel{\circ}{B}_{1} \Phi_{k}, \Phi_{k}\right)_{\mathcal{P}[m, n]}=\frac{i \sqrt[4]{5-a}}{\sqrt{|m|} \sqrt{10}}\left(\left(\sqrt{a}-\frac{\sqrt{5-a}}{2}\right) \frac{\bar{u}}{\sqrt{2}} p_{0}\right. \\
\left.-\sqrt{a} \alpha_{1} \mu \exp \left(\frac{\sqrt{|m|}(\sqrt{a}-1) \sqrt{5} \bar{u}}{2 \sqrt[4]{5-a}}+\left(-1+\frac{5-3 \sqrt{a}}{\sqrt{5-a}}\right) \frac{\bar{u}^{2}}{4}\right) H_{k}^{\prime}\left(\frac{\bar{u}}{\sqrt{2}}\right), p_{0}\right)_{\mathcal{P}[m, n]} \\
+O\left(|m|^{-1}\right)=O\left(|m|^{-1}\right) .
\end{array}
$$

Finally, taking into account (195) and (199), we have

$$
\begin{aligned}
\left(\stackrel{\circ}{B}_{2} \Phi_{k}, \Phi_{k}\right)_{\mathcal{P}[m, n]}=\left(\frac{(a-1)}{4}\left(p_{0}+\frac{p_{1}}{\sqrt{|m|}}\right), p_{0}+\frac{p_{1}}{\sqrt{|m|}}\right)_{\mathcal{P}[m, n]} & O\left(\frac{1}{|m|}\right) \\
& =\frac{a-1}{4}+O\left(\frac{1}{|m|}\right), \\
\left(\stackrel{\circ}{B}_{3} \Phi_{k}, \Phi_{k}\right)_{\mathcal{P}[m, n]}=\left(\frac{(a+1)}{4}\left(p_{0}+\frac{p_{1}}{\sqrt{|m|}}\right), p_{0}+\frac{p_{1}}{\sqrt{|m|}}\right)_{\mathcal{P}[m, n]} & +O\left(\frac{1}{|m|}\right) \\
& =\frac{a+1}{4}+O\left(\frac{1}{|m|}\right) .
\end{aligned}
$$

From (194) and the Taylor formula, we have

Theorem 6. Let $F\left(\stackrel{\circ}{B}_{0}, \stackrel{\circ}{B}_{1}, \stackrel{\circ}{B}_{2}, \stackrel{\circ}{B}_{3}\right)$ be an operator, where $F\left(b_{0}, b_{1}, b_{2}, b_{3}\right)$ is a polynomial such that $F(0,0,(a-1) / 4,(a+1) / 4) \neq 0$, and the operators $\stackrel{\circ}{B}_{0}, \stackrel{\circ}{B}_{1}, \stackrel{\circ}{B}_{2}, \stackrel{\circ}{B}_{3}$ are Weyl-ordered. Then, as $|m| \rightarrow \infty$, the formula for the quantum averages with accuracy $O\left(|m|^{-1}\right)$ is of the form

$$
\left(F\left(\stackrel{\circ}{B}_{0}, \stackrel{\circ}{B}_{1}, \stackrel{\circ}{B}_{2}, \stackrel{\circ}{B}_{3}\right) \Phi_{k}, \Phi_{k}\right)_{\mathcal{P}[m, n]}=F\left(0,0, \frac{a-1}{4}, \frac{a+1}{4}\right)+O\left(|m|^{-1}\right) .
$$

Notice that if in (27) we set $\hbar=1 / n$ and use the substitution $c^{2}=1 / a$, then $(200)$ implies the formula

$$
\left(F\left(\stackrel{\circ}{B}_{0}, \stackrel{\circ}{B}_{1}, \stackrel{\circ}{B}_{2}, \stackrel{\circ}{B}_{3}\right) \Phi_{k}, \Phi_{k}\right)_{\mathcal{P}[m, n]}=F\left(0,0, \frac{1-c^{2}}{4}, \frac{1+c^{2}}{4}\right)+O\left(n^{-1}\right), \quad n \rightarrow \infty .
$$

Thus, the asymptotics (47) is valid.

Nontrivial corrections in the formula for the averages appear in the terms of order $|m|^{-1}$. To obtain these corrections, one needs to use the next term $p_{2} /|m|$ in the expansion (112). If the correction of order $|m|^{-2}$ in the formula (75) equals

$$
\tilde{\xi}_{k}^{(2)}=\frac{3(15+a)}{2(5-a)}\left(k+\frac{1}{2}\right)^{2}+\frac{5(17-a)}{8(5-a)},
$$

then there is a function

$$
\begin{gathered}
p_{2}=\frac{\alpha_{1} \mu}{(5-a)^{3 / 2}} \exp \left(\frac{\sqrt{|m|}(\sqrt{a}-1) \beta \bar{u}}{2}+\left(-1+\frac{(5-3 \sqrt{a})}{\sqrt{5-a}}\right) \frac{\bar{u}^{2}}{4}\right)\left\{\left\{\frac{5}{36}[7 a-80 \sqrt{a}\right.\right. \\
+145+12(2 \sqrt{a}-5) \sqrt{5-a}]\left(\frac{\bar{u}}{\sqrt{2}}\right)^{6}+\frac{1}{48}\left[261 a-1365-120(5-a)\left(k+\frac{1}{2}\right)\right. \\
+8(25-\sqrt{a}) \sqrt{5-a}]\left(\frac{\bar{u}}{\sqrt{2}}\right)^{4}+\frac{1}{32}\left[-107 a+475-6(15+a)\left(k+\frac{1}{2}\right)\right]\left(\frac{\bar{u}}{\sqrt{2}}\right)^{2} \\
\left.+\alpha_{2}\right\} H_{k}\left(\frac{\bar{u}}{\sqrt{2}}\right)+\left\{\frac{5}{3}(5-2 \sqrt{a}) \sqrt{5-a}\left(\frac{\bar{u}}{\sqrt{2}}\right)^{5}+\frac{(455-87 a)}{16}\left(\frac{\bar{u}}{\sqrt{2}}\right)^{3}\right. \\
\left.\left.+\frac{3}{16}(15+a)\left(k+\frac{1}{2}\right)\left(\frac{\bar{u}}{\sqrt{2}}\right)\right\} H_{k}^{\prime}\left(\frac{\bar{u}}{\sqrt{2}}\right)\right\},
\end{gathered}
$$


such that the asymptotics near $\bar{z}=1$ matches the WKB approximation $\Phi_{-}^{W K B}(\bar{z})$. The constant $\alpha_{2}$ in (202) can be found from the normalizing condition (29).

Similar to Theorem 6 , one can prove

Theorem 7. Let $F\left(\stackrel{\circ}{B}_{0}, \stackrel{\circ}{B}_{1}, \stackrel{\circ}{B}_{2}, \stackrel{\circ}{B}_{3}\right)$ be an operator, where $F\left(b_{0}, b_{1}, b_{2}, b_{3}\right)$ is a polynomial such that $F(0,0,(a-1) / 4,(a+1) / 4) \neq 0$, and the operators $\stackrel{\circ}{B}_{0}, \stackrel{\circ}{B}_{1}, \stackrel{\circ}{B}_{2}, \stackrel{\circ}{B}_{3}$ are Weyl-ordered. Then, as $|m| \rightarrow \infty$, the formula for the quantum averages with accuracy $O\left(|m|^{-3 / 2}\right)$ is of the form

$$
\begin{gathered}
\left(F\left(\stackrel{\circ}{B}_{0}, \stackrel{\circ}{B}_{1}, \stackrel{\circ}{B}_{2}, \stackrel{\circ}{B}_{3}\right) \Phi_{k}, \Phi_{k}\right)=F+\frac{\partial F}{\partial b_{0}}\left(\stackrel{\circ}{B}_{0} \Phi_{k}, \Phi_{k}\right)+\frac{\partial F}{\partial b_{1}}\left(\stackrel{\circ}{B}_{1} \Phi_{k}, \Phi_{k}\right) \\
+\frac{\partial F}{\partial b_{2}}\left[\left(\stackrel{\circ}{B}_{2} \Phi_{k}, \Phi_{k}\right)-\frac{a-1}{4}\right]+\frac{\partial F}{\partial b_{3}}\left[\left(\stackrel{\circ}{B}_{3} \Phi_{k}, \Phi_{k}\right)-\frac{a+1}{4}\right] \\
+\frac{1}{2} \frac{\partial^{2} F}{\partial b_{0}^{2}}\left(\left(\stackrel{\circ}{B}_{0}\right)^{2} \Phi_{k}, \Phi_{k}\right)+\frac{1}{2} \frac{\partial^{2} F}{\partial b_{1}^{2}}\left(\left(\stackrel{\circ}{B}_{1}\right)^{2} \Phi_{k}, \Phi_{k}\right)+\frac{\partial^{2} F}{\partial b_{0} \partial b_{1}}\left(\frac{\stackrel{\circ}{B}_{0} \stackrel{\circ}{B}_{1}+\stackrel{\circ}{B}_{1} \stackrel{\circ}{0}_{0}}{2} \Phi_{k}, \Phi_{k}\right) \\
+O\left(|m|^{-3 / 2}\right) .
\end{gathered}
$$

Here

$$
\begin{aligned}
& \left(\stackrel{\circ}{B}_{0} \Phi_{k}, \Phi_{k}\right)=-\frac{\sqrt{5-a}}{|m| \sqrt{a}} \frac{\Sigma_{1}(a)}{\Sigma_{0}(a)}+O\left(|m|^{-3 / 2}\right), \\
& \left(\stackrel{\circ}{B}_{1} \Phi_{k}, \Phi_{k}\right)=\frac{i \sqrt{a}}{|m|}\left(1-\frac{2 \sqrt{a}}{\sqrt{5-a}}\right) \frac{\Sigma_{1}(a)}{\Sigma_{0}(a)}+O\left(|m|^{-3 / 2}\right), \\
& \left(\stackrel{\circ}{B}_{2} \Phi_{k}, \Phi_{k}\right)=\frac{a-1}{4}+\frac{1}{|m|}\left[\frac{3 \sqrt{a}}{10}-\frac{\sqrt{5-a}}{5}\left(k+\frac{1}{2}\right)\right]-\frac{3(a-1)}{|m| 4 \sqrt{5-a}} \frac{\Sigma_{2}(a)}{\Sigma_{0}(a)}+O\left(|m|^{-3 / 2}\right), \\
& \left(\stackrel{\circ}{B}_{3} \Phi_{k}, \Phi_{k}\right)=\frac{a+1}{4}+\frac{1}{5|m|}\left[\sqrt{a}+\sqrt{5-a}\left(k+\frac{1}{2}\right)\right]-\frac{a-1}{|m| 2 \sqrt{5-a}} \frac{\Sigma_{2}(a)}{\Sigma_{0}(a)}+O\left(|m|^{-3 / 2}\right), \\
& \left(\left(\stackrel{\circ}{B}_{0}\right)^{2} \Phi_{k}, \Phi_{k}\right)=-\frac{4}{5|m|}\left[\sqrt{a}+\sqrt{5-a}\left(k+\frac{1}{2}\right)\right]+\frac{2(a-1)}{|m| \sqrt{5-a}} \frac{\Sigma_{2}(a)}{\Sigma_{0}(a)}+O\left(|m|^{-3 / 2}\right), \\
& \left(\left(\stackrel{\circ}{B}_{1}\right)^{2} \Phi_{k}, \Phi_{k}\right)=\frac{\sqrt{a} \sqrt{5-a}}{20|m|}\left[\sqrt{5-a}+4 \sqrt{a}\left(k+\frac{1}{2}\right)\right]-\frac{(a-1) \sqrt{5-a}}{8|m|} \frac{\Sigma_{2}(a)}{\Sigma_{0}(a)} \\
& +O\left(|m|^{-3 / 2}\right) \\
& \left(\frac{\stackrel{\circ}{B}_{0} \stackrel{\circ}{B}_{1}+\stackrel{\circ}{B}_{1} \stackrel{\circ}{B}_{0}}{2} \Phi_{k}, \Phi_{k}\right)=\frac{i}{20|m|}\left[3 a+5+8 \sqrt{a} \sqrt{5-a}\left(k+\frac{1}{2}\right)\right]-\frac{i(a-1)}{2|m|} \frac{\Sigma_{3}(a)}{\Sigma_{0}(a)} \\
& +O\left(|m|^{-3 / 2}\right), \\
& \Sigma_{j}(a)=\int_{\mathbb{R}^{2}} \theta(t, r, a) \sigma_{j}(t, r, a)\left|H_{k}(t+i r)\right|^{2} d t d r, \quad j=1,2,3,
\end{aligned}
$$

where $\theta$ is given by (176), and

$$
\sigma_{1}=t^{2}, \sigma_{2}=t^{2}+r^{2}, \sigma_{3}=\frac{\sqrt{5-a}}{2 \sqrt{a}} t^{2}+\frac{2 \sqrt{a}}{\sqrt{5-a}} r^{2} .
$$

In (203), the values of $F$ and of its derivatives are computed at the point $(0,0$, $(a-1) / 4,(a+1) / 4)$, and the scalar product is taken in the space $\mathcal{P}[m, n]$.

Let us apply (201) to find the series of asymptotic eigenvalues of the operator (1) near the lower boundaries of the spectral clusters forming around the eigenvalues of the unperturbed operator. Since the transformation (26) is unitary, the numbers $\eta_{k}$ from (13) can be computed by the formula

$$
\eta_{k}=\left(g_{00}(\boldsymbol{B}) \Phi_{k}, \Phi_{k}\right)_{\mathcal{P}[m, n]}
$$


where the operator $g_{00}(\boldsymbol{B})$ is given by $(18)$. We then have

$$
\begin{aligned}
& \eta_{k}=- \frac{1}{3}\left[4\left\{108\left(\frac{1-c^{2}}{4}\right)^{2}+239\left(\frac{1+c^{2}}{4}\right)^{2}-308\left(\frac{1-c^{2}}{4}\right)\left(\frac{1+c^{2}}{4}\right)\right\}\right. \\
&\left.+4\left(-66+100 c^{2}\right) \frac{1-c^{2}}{4}+4\left(65-130 c^{2}\right) \frac{1+c^{2}}{4}-127 c^{2}+72 c^{4}\right]+O\left(n^{-1}\right) \\
&=- \frac{1}{3}\left[\frac{35}{4}+\frac{79}{2} c^{2}+\frac{23}{4} c^{4}\right]+O\left(n^{-1}\right)=-\frac{1}{12 n^{4}}\left[35 n^{4}+158 m^{2} n^{2}+23 m^{4}\right] \\
&+O\left(n^{-1}\right), \quad n \rightarrow \infty .
\end{aligned}
$$

Furthermore, by (50) and (193),

$$
\begin{aligned}
\xi_{k}=c^{2}\left[\frac{1}{c^{2}}+1+\frac{4}{|m|}\right. & \left.\sqrt{5-\frac{1}{c^{2}}}\left(k+\frac{1}{2}\right)\right]+O\left(n^{-2}\right) \\
= & \frac{n^{2}+m^{2}}{n^{2}}+\frac{4}{n^{2}} \sqrt{5 m^{2}-n^{2}}\left(k+\frac{1}{2}\right)+O\left(n^{-2}\right), \quad n \rightarrow \infty
\end{aligned}
$$

and, therefore,

$$
\begin{aligned}
& 2 \eta_{k}+5 \xi_{k}^{2}=- \frac{1}{6 n^{4}}\left(35 n^{4}+158 m^{2} n^{2}+23 m^{4}\right)+\frac{5}{n^{4}}\left(n^{4}+2 m^{2} n^{2}+m^{4}\right) \\
&+O\left(n^{-1}\right)=-\frac{1}{6 n^{4}}\left(5 n^{4}+98 n^{2} m^{2}-7 m^{4}\right)+O\left(n^{-1}\right), \quad n \rightarrow \infty .
\end{aligned}
$$

Now substitute (204) and (205) in (14), and add to the spectrum the term $\varepsilon m$, reflecting the presence in (1) of a magnetic field of order $\varepsilon$. We then have

Theorem 8. Suppose $1 \ll n \lesssim \varepsilon^{-7 / 2}$ and $5^{-1 / 2} n<|m|<n$. Then, near the lower boundaries of the spectral clusters, there is a series of eigenvalues of the operator (1) with the following asymptotics:

$$
\begin{aligned}
\mathcal{E}_{k}=- & \frac{1}{4 n^{2}}+\varepsilon m+\frac{1}{2} \varepsilon^{2} n^{2}\left(n^{2}+m^{2}\right)+\varepsilon^{2} n^{2}(2 k+1) \sqrt{5 m^{2}-n^{2}} \\
& \quad-\frac{1}{24} \varepsilon^{4} n^{6}\left(5 n^{4}+98 m^{2} n^{2}-7 m^{4}\right)+O\left(n^{-9 / 2}\right), \quad n \rightarrow \infty,=0,1,2, \ldots .
\end{aligned}
$$

Notice that the series (206) coincides with the series found in 9]. The formula (206), describing the splitting of the spectrum (i.e., the Zeeman effect [38]), has been extended to the terms of order four in the magnetic field. The corresponding asymptotic eigenfunctions can be obtained from the polynomials $\Phi_{k}$, given by (57), by using the coherent transformation $\mathfrak{H}$ and the de-averaging transformation $\boldsymbol{U}$ (see (23), (11)).

\section{ACKNOWLEDGEMENT}

The author is grateful to M. V. Karasev for his attention to this problem and for valuable questions and remarks.

\section{REFERENCES}

[1] H. Friedrich and D. Wintgen, The hydrogen atom in a uniform magnetic field - an example of chaos, Phys. Rep. 183 (1989), no. 2, 37-79. MR1024519(90j:81279)

[2] V. S. Lisitsa, New results on the Stark and Zeeman effects in the hydrogen atom, Uspekhi Fiz. Nauk 153 (1987), no. 3, 379-421; English transl., Soviet Phys. Uspekhi 30 (1987), no. 11, 927-951. $\operatorname{MR} 966185(89 \mathrm{i}: 81148)$

[3] J. E. Avron, I. W. Herbst, and B. Simon, Schrödinger operators with magnetic fields. III. Atoms in homogeneous magnetic field, Comm. Math. Phys. 79 (1981), no. 4, 529-572. MR623966 (85h:81084)

[4] E. A Solov'ëv, The hydrogen atom in a weak magnetic field, Zh. Eksp. Teor. Fiz. 82 (1982), no. 6, 1762-1771; English transl., Sov. Phys. JETP 55 (1982), no. 6, 1017-1022 
[5] D. R. Herrick, Symmetry of the quadratic Zeeman effect for hydrogen, Phys. Rev. A (3) 26 (1982), no. 1, 323-329. MR668295 (83i:81075)

[6] D. Delande and J. C. Gay, Group theory applied to the hydrogen atom in a strong magnetic field. Derivation of the effective diamagnetic Hamiltonian, J. Phys. B 17 (1984), no. 11, L335-L340. MR.748791 (85e:81133)

[7] V. V. Belov and J. L. Volkova, Investigation of the Zeeman effect in quasiclassical trajectorycoherent approximation, Preprint no. 35, Tomsk Scientific Centre, Siberian Division, AS USSR, 1991.

[8] V. V. Belov, V. M. Olivé, and J. L. Volkova, The Zeeman effect for the "anisotropic hydrogen atom" in the complex WKB approximation. I. Quantization of closed orbits for the Pauli operator with spin-orbit interaction, J. Phys. A 28 (1995), no. 20, 5799-5810. MR1364760 (96m:81053a)

[9] M. V. Karasev and E. M. Novikova, Representation of exact and quasiclassical eigenfunctions in terms of coherent states. The hydrogen atom in a magnetic field, Teoret. Mat. Fiz. 108 (1996), no. 3, 339-387; English transl., Theoret. and Math. Phys. 108 (1996), no. 3, 1119-1159. MR1430079 (98b:81083)

[10] M. V. Karasev, Birkhoff resonances and quantum ray method, Proc. Intern. Seminar "Days of Diffraction- 2004", St. Petersburg University and Steklov Math. Institute, St. Petersburg, 2004, $114-126$.

[11] M. V. Karasev, Noncommutative algebras, nano-structures, and quantum dynamics generated by resonances. I, Quantum Algebras and Poisson Geometry in Mathematical Physics, Amer. Math. Soc., Providence, RI, 2005, 1-18; MR2180511 (2006h:81143) II, Adv. Stud. Contemp. Math. (Kyungshang) 11 (2005), no. 1, 33-56; III, Russ. J. Math. Phys. 13 (2006), no. 2, 131-150. MR:2151283 (2006h:81144) MR2262820 (2007h:81122)

[12] M. V. Karasev and E. M. Novikova, An algebra with quadratic commutation relations for an axially perturbed Coulomb-Dirac field, Teoret. Mat. Fiz. 141 (2004), no. 3, 424-454; English transl., Theoret. and Math. Phys. 141 (2004), no. 3, 1698-1724. MR2141137 (2006e:81120)

[13] M. V. Karasev and E. M. Novikova, An algebra with polynomial commutation relations for the Zeeman effect in the Coulomb-Dirac field, Teoret. Mat. Fiz. 142 (2005), no. 1, 127-147; English transl., Theoret. and Math. Phys. 142 (2005), no. 1, 109-127. MR2137424 (2006b:81134)

[14] M. V. Karasev and E. M. Novikova, An algebra with polynomial commutation relations for the Zeeman-Stark effect in the hydrogen atom, Teoret. Mat. Fiz. 142 (2005), no. 3, 530-555; English transl., Theoret. and Math. Phys. 142 (2005), no. 3, 447-469. MR2165905 (2006d:81348)

[15] A. V. Pereskokov, Asymptotics near the boundaries of spectral clusters, International Conference in memory of I. G. Petrovsky (23rd joint meeting of the MMS and the Petrovsky Seminar), Abstracts of talks, MGU Publishing, Moscow, 2011, 260-261.

[16] A. V. Pereskokov, Asymptotics of the spectrum and quantum averages near the boundaries of spectral clusters for perturbed two-dimensional oscillators, Mat. zam. 92 (2012), no. 4, 583-596; English transl., Math Notes 92 (2012), no. 4, 532-543.

[17] V. M. Babich and V. S. Buldyrev, Short-wavelength diffraction theory. Asymptotic methods, Nauka, Moscow, 1972; English transl., Springer Series on Wave Phenomena, 4, Springer-Verlag, Berlin, 1991. MR 1245488(94f:78004)

[18] V. P. Maslov, The complex WKB method for nonlinear equations. Nauka, Moscow, 1977; English transl., Progress in Physics, 16. Birkhäuser Verlag, Basel, 1994. MR1306505 (95m:58126)

[19] M. V. Karasev and V. P. Maslov, Asymptotic and geometric quantization, Uspekhi Mat. Nauk 39 (1984), no. 6(240), 115-173; English transl., Russian Math. Surveys 39 (1985), no. 6, 133-205. $\operatorname{MR} 771100$ (86m:58064)

[20] A Weinstein, Asymptotics of eigenvalue clusters for the Laplacian plus a potential, Duke Math. J. 44 (1977), no. 4, 883-892. MR0482878(58:2919)

[21] M. V. Karasev and E. M. Novikova, Non-Lie permutation relations, coherent states, and quantum embedding, Coherent transform, quantization, and Poisson geometry, 1-202, Amer. Math. Soc. Transl. Ser. 2, 187, Amer. Math. Soc., Providence, RI, 1998. MR.1728668 (2001i:81116)

[22] A. Erdélyi, W. Magnus, F. Oberhettinger, and F. Tricomi, Higher transcendental functions, vols. I, II, McGraw-Hill Book Company, Inc., New York-Toronto-London, 1953. MR0058756 (15:419i)

[23] A. M. Perelomov, Generalized coherent states and their applications, Nauka, Moscow, 1987; English transl., Texts and Monographs in Physics, Springer-Verlag, Berlin, 1986. MR858831 (87m:22035)

[24] V. V. Golubev, Lectures on analytic theory of differential equations, Gostekhisdat, Moscow, 1950. (Russian) MR0014552(7:301a)

[25] S. Yu. Slavyanov and W. Lay, Special functions. A unified theory based on singularities, Nevski Dialekt, St. Petersburg, 2002; English transl., Oxford Mathematical Monographs. Oxford Science Publications. Oxford University Press, Oxford, 2000. MR.1858237 (2004e:33003) 
[26] P. A. M. Dirac, Quantum electrodynamics, Comm. Dublin Inst. Adv. Stud., Ser. A 1 (1943), 1-36. MR0013042(7:100e)

[27] M. V. Fedoryuk, Asymptotic methods for linear ordinary differential equations, Nauka, Moscow, 1983. (Russian) MR732787 (85m:34001)

[28] F. Olver, Asymptotics and special functions, A K Peters, Ltd., Wellesley, MA, 1997. MR1429619 (97i:41001)

[29] M. A. Lavrentév and B. V. Shabat, Methods of the theory of functions of a complex variable, Nauka, Moscow, 1987. (Russian) MR1087298 (91k:30003)

[30] I. S. Gradshteyn and I. M. Ryzhik, Table of integrals, series, and products, Nauka, Moscow, 1975; English transl., Academic Press [Harcourt Brace Jovanovich, Publishers], New York-LondonToronto, Ont., 1980. MR582453 (81g:33001)

[31] J. Jenkins, Univalent functions and conformal mappings, Springer-Verlag, Berlin-GöttingenHeidelberg, 1958. MR0096806 (20:3288)

[32] M. Abramowitz and I. Stegun, Handbook of mathematical functions with formulas, graphs, and mathematical tables, National Bureau of Standards Applied Mathematics Series, 55, Washington, D.C., 1964. MR0167642 (29:4914)

[33] M. V. Fedoryuk, Asymptotics: integrals and series, Nauka, Moscow, 1987. (Russian) MR950167 $(89 \mathrm{j}: 41045)$

[34] P. A. Braun, WKB method for three-term recursion relations and quasienergies of an anharmonic oscillator, Teoret. Mat. Fiz. 37 (1978), no. 3, 355-370; English transl., Theoret. and Math. Phys. 37 (1978), no. 3, 1070-1081. MR524700 (80b:81020)

[35] A. B. Vasil'eva, The correspondence between certain properties of the solutions of linear difference systems and systems of ordinary linear differential equations, Trudy Sem. Teor. Differencial. Uravneniís Otklon. Argumentom, Univ. Druzhby Narodov Patrisa Lumumby 5 (1967), 21-44. MR0227645(37:3229)

[36] V. P. Maslov and M. V. Fedoryuk, Quasiclassical approximation for the equations of quantum mechanics, Nauka, Moscow, 1976. (Russian) MR 0461590 (57:1575)

[37] V. P. Maslov, Complex Markov chains and the Feynman path integral for nonlinear equations, Nauka, Moscow, 1976. (Russian) MR0479126 (57:18574)

[38] L. I. Schiff, Quantum mechanics, McGraw-Hill, 1965.

Moscow Power Engineering Institute, Moscow Institute of Electronics and Mathematics of the Higher School of Economics - National Research University

E-mail address: pereskokov62@mail.ru 